\title{
Genetic mechanisms of critical illness in COVID-19
}

https://doi.org/10.1038/s41586-020-03065-y

Received: 27 September 2020

Accepted: 30 November 2020

Published online: 11 December 2020

Check for updates
A list of authors and their affiliations appears at the end of the paper.

Host-mediated lung inflammation is present ${ }^{1}$, and drives mortality ${ }^{2}$, in the critical illness caused by coronavirus disease 2019 (COVID-19). Host genetic variants associated with critical illness may identify mechanistic targets for therapeutic development ${ }^{3}$. Here we report the results of the GenOMICC (Genetics Of Mortality In Critical Care) genome-wide association study in 2,244 critically ill patients with COVID-19 from 208 UK intensive care units. We have identified and replicated the following new genome-wide significant associations: on chromosome 12q24.13 ( $r$ 10735079, $P=1.65 \times 10^{-8}$ ) in a gene cluster that encodes antiviral restriction enzyme activators (OAS1, OAS2 and OAS3); on chromosome 19p13.2 (rs74956615, $P=2.3 \times 10^{-8}$ ) near the gene that encodes tyrosine kinase 2 (TYK2); on chromosome 19p13.3 ( $r 2109069, P=3.98 \times 10^{-12}$ ) within the gene that encodes dipeptidyl peptidase 9 (DPP9); and on chromosome 21q22.1 ( $\left.\mathrm{rs} 2236757, P=4.99 \times 10^{-8}\right)$ in the interferon receptor gene IFNAR2. We identified potential targets for repurposing of licensed medications: using Mendelian randomization, we found evidence that low expression of IFNAR2, or high expression of $T Y K 2$, are associated with life-threatening disease; and transcriptome-wide association in lung tissue revealed that high expression of the monocyte-macrophage chemotactic receptor CCR2 is associated with severe COVID-19. Our results identify robust genetic signals relating to key host antiviral defence mechanisms and mediators of inflammatory organ damage in COVID-19. Both mechanisms may be amenable to targeted treatment with existing drugs. However, large-scale randomized clinical trials will be essential before any change to clinical practice.
As critical illness in patients with COVID-19 is caused, in part, by inflammatory injuries that affect the lungs and lung blood vessels ${ }^{1}$, there are at least two distinct biological components to the mortality risk: susceptibility to viral infection and propensity to develop harmful pulmonary inflammation. Susceptibility to life-threatening infections ${ }^{4}$ and immune-mediated diseases are both strongly heritable. In particular, susceptibility to respiratory viruses ${ }^{5}$ such as influenza $^{6}$ is heritable and known to be associated with specific genetic variants ${ }^{7}$. In the case of COVID-19, one genetic locus-on chromosome 3 21.31-has been repeatedly associated with hospitalization ${ }^{8,9}$. As with other virus-associated disease ${ }^{10}$, there are several examples of loss-of-function variants affecting essential immune processes that lead to severe disease in young people, such as $T L R 7^{11}$ and some genes implicated in type 1 interferon signalling, including the receptor subunit IFNAR2 $2^{12}$. Genome-wide studies have the potential to reveal previously undescribed molecular mechanisms of critical illness in patients with COVID-19, which may provide therapeutic targets to modulate the host immune response to promote survival ${ }^{3}$.

Strong evidence indicates that critical illness caused by COVID-19 is qualitatively different from mild or moderate disease, even among hospitalized patients. There are multiple distinct disease phenotypes with differing patterns of symptom ${ }^{13}$ and marked differential responses to immunosuppressive therapy ${ }^{2}$. In patients without respiratory failure, there is a trend indicating that treatment with corticosteroids is harmful, whereas among patients with critical respiratory failure, there is a substantial benefit ${ }^{2}$. On this basis, we consider patients with critical COVID-19 respiratory failure to have a distinct pathophysiology.

In the UK, the group of patients admitted to critical care is relatively homogeneous, with profound hypoxaemic respiratory failure being the archetypal presentation ${ }^{14}$. The active disease process in these patients is markedly responsive to corticosteroid therapy ${ }^{15}$ and is characterized by pulmonary inflammation including diffuse alveolar damage, influx of monocytes and macrophagess, mononuclear cell pulmonary artery vasculitis and microthrombus formation ${ }^{1,16}$.

Host-directed therapies have long been an aim for the treatment of the severe disease caused by respiratory viruses ${ }^{17}$. Identification of genetic loci associated with susceptibility to COVID-19 may lead to specific targets for the development or repurposing of drugs ${ }^{3}$.

The GenOMICC (Genetics Of Mortality In Critical Care, https:// genomicc.org/) study has been recruiting patients with critical illness syndromes, including influenza, sepsis and emerging infections, for 5 years. To better understand the host mechanisms that lead to life-threatening disease in patients with COVID-19, we performed a genome-wide association study (GWAS) comparing critically ill patients with COVID-19 with controls from population genetic studies in the UK.

\section{Characteristics of participants}

Critically ill cases were recruited through the GenOMICC study in 208 UK intensive care units and hospitalized cases were recruited through 
Table 1 | Lead variants from independent genome-wide significant regions

\begin{tabular}{|c|c|c|c|c|c|c|c|c|c|c|c|}
\hline SNP & Chr.: pos. & Risk & Alt. & $\mathbf{R A F}_{\text {gcc }}$ & $\mathbf{R A F}_{\text {ukb }}$ & OR & $\mathbf{C l}$ & $P_{\text {gcc.ukb }}$ & $P_{\text {gcc.gs }}$ & $P_{\text {gcc.100k }}$ & Locus \\
\hline rs73064425 & 3: 45,901,089 & $\mathrm{T}$ & C & 0.15 & 0.07 & 2.1 & $1.88-2.45$ & $4.8 \times 10^{-30}$ & $2.9 \times 10^{-27}$ & $3.6 \times 10^{-32}$ & LZTFL1 \\
\hline rs9380142 & $6: 29,798,794$ & A & G & 0.74 & 0.69 & 1.3 & $1.18-1.43$ & $3.2 \times 10^{-8}$ & 0.00091 & $1.8 \times 10^{-8}$ & $H L A-G$ \\
\hline rs143334143 & $6: 31,121,426$ & $A$ & $G$ & 0.12 & 0.07 & 1.8 & $1.61-2.13$ & $8.8 \times 10^{-18}$ & $2.6 \times 10^{-24}$ & $5.8 \times 10^{-18}$ & $\mathrm{CCHCR} 1$ \\
\hline rs3131294 & $6: 32,180,146$ & $G$ & $A$ & 0.9 & 0.86 & 1.5 & $1.28-1.66$ & $2.8 \times 10^{-8}$ & $1.3 \times 10^{-10}$ & $2.3 \times 10^{-8}$ & $\mathrm{NOTCH} 4$ \\
\hline rs10735079 & $12: 113,380,008$ & A & G & 0.68 & 0.63 & 1.3 & $1.18-1.42$ & $1.6 \times 10^{-8}$ & $2.8 \times 10^{-9}$ & $4.7 \times 10^{-6}$ & OAS1-OAS3 \\
\hline rs2109069 & 19: 4,719,443 & A & G & 0.38 & 0.32 & 1.4 & $1.25-1.48$ & $4 \times 10^{-12}$ & $4.5 \times 10^{-7}$ & $2.4 \times 10^{-8}$ & DPP9 \\
\hline rs74956615 & 19: 10,427,721 & $A$ & $\mathrm{~T}$ & 0.079 & 0.05 & 1.6 & $1.35-1.87$ & $2.3 \times 10^{-8}$ & $2.2 \times 10^{-13}$ & $3.9 \times 10^{-6}$ & TYK2 \\
\hline rs2236757 & $21: 34,624,917$ & $A$ & $G$ & 0.34 & 0.28 & 1.3 & $1.17-1.41$ & $5 \times 10^{-8}$ & $8.9 \times 10^{-5}$ & $8.3 \times 10^{-7}$ & IFNAR2 \\
\hline
\end{tabular}

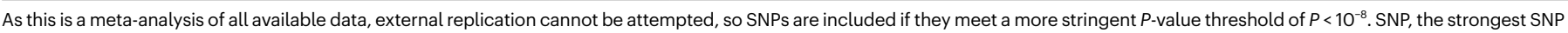
in the locus. Chr.: pos., chromosome and position of the top SNP (build 37); Risk, risk allele; Alt., alternative allele; RAF, risk allele frequency; OR, effect size (odds ratio) of the risk allele in the

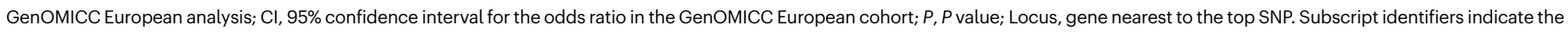
cohorts used for cases (gcc, GenOMICC European cohort) and controls (ukb, UK Biobank; gs, Generation Scotland; 100k, 100,000 Genomes Project).

the International Severe Acute Respiratory Infection Consortium (ISARIC) Coronavirus Clinical Characterization Consortium (4C) study.

DNA was extracted from whole blood and array-based genome-wide genotypes of good quality were obtained for 2,734 unique individuals (Methods). Genetic ancestry was inferred using principal component analyses and individuals from the 1000 Genomes Project were included as population references (Methods). After quality control and matching to ancestry groups, 2,244 individuals were included in the GWAS for analysis. Clinical and demographic features of these cases are shown in Extended Data Table1. Additional clinical details for a subset of 1,069 cases for whom additional data were available are presented in Supplementary Figs. 7-13 and Supplementary Table 2. Cases were representative of critically ill patients with COVID-19 in the UK population ${ }^{14}$. Imputation in this multi-ancestry cohort was performed using the TOPMed reference panel.

Ancestry-matched control individuals were selected from the large population-based cohort of UK Biobank (five controls were included for each case). Controls with a known positive COVID-19 test were excluded. The inevitable presence of individuals in the control group, who may exhibit the critical illness phenotype if exposed toSARS-CoV-2 is expected to bias any associations towards the null hypothesis. GWAS was carried out separately for each ancestry group using logistic regression in PLINK and accounting for age, sex, deprivation decile based on the individual's residential postal code and principal components of ancestry. In addition to several standard filters to minimize spurious associations (Methods), whole-genome sequencing analysis of a subset of 1,613 cases was used to filter out variants that are likely to have been badly called or imputed; 83,937 out of the 4,469,187 imputed variants that passed other quality-control filters after GWAS were thus removed. There was a high level of residual inflation in the South Asian and East Asian ancestry groups, rendering results in these subgroups unreliable (Extended Data Fig. 1, Supplementary Fig. 4). The largest ancestry group contained 1,676 individuals of European descent; this group was used for the primary analyses presented below.

\section{GWAS results}

In the primary analysis (cases of European descent from GenOMICC versus controls from the UK Biobank), after linkage-disequilibrium-based clumping, 15 independent association signals were genome-wide significant at $P<5 \times 10^{-8}$ (Supplementary Fig. 1). Eight of these signals were successfully validated in GWAS analyses using two independent population genetic studies (100,000 Genomes Project and Generation Scotland: Scottish Family Health Study, hereafter Generation Scotland) as controls (Table 1) and were therefore taken forward for replication. A sex-specific GWAS among this group found no sex-specific associations (Supplementary Table 1). A trans-ethnic meta-analysis did not reveal any additional associations (Supplementary Fig. 3).

\section{Replication}

As no study of critical illness in patients with COVID-19 of sufficient size is available, replication was carried out in a meta-analysis of data from 2,415 hospitalized patients with COVID-19 and 477,741 population controls from the COVID-19 Host Genetics Initiative (HGI, individuals of mixed ancestry from which cases and controls of the UK Biobank were excluded) and 1,128 cases and 679,531 controls of European ancestry from the 'broad respiratory phenotype' study of 23andMe Inc, which includes cases that were reported as having been placed on a ventilator, administered oxygen or having had pneumonia compared with control individuals who did not report COVID-19-positive tests. In addition to the locus on chromosome 3 that has previously been reported (rs73064425, odds ratio $=2.14$, discovery $P=4.77 \times 10^{-30}$ ), we found robust replication for previously undescribed associations in four loci from GenOMICC: a locus on chromosome 12 in the OAS gene cluster (rs10735079, odds ratio $=1.3$, discovery $P=1.65 \times 10^{-8}$ ), near $T Y K 2$ on chromosome 19 ( rs74956615, odds ratio $=1.6$, discovery $P=2.3 \times 10^{-8}$ ), in $D P P 9$ on chromosome 19 (rs2109069, odds ratio $=1.36$, discovery $P=3.98 \times 10^{-12}$ ) and a locus on chromosome 21, which contains the gene IFNAR2(rs2236757, odds ratio $=1.28$, discovery $P=4.99 \times 10^{-8}$ ) (Fig. 1, Extended Data Table2).

Three variants, all in a region of chromosome 6 for which population stratification is difficult to control (the major histocompatibility complex) did not replicate (Extended Data Table2,Supplementary Fig. 2). Further studies will be required to determine whether these associations are real.

To increase power for exploratory analyses, inverse-variance meta-analysis was performed between critically ill patients of European descent from GenOMICC ( $n=1,676$ cases, $n=8,380$ controls), hospitalized patients with COVID-19 versus population controls (B2, version 2) without UK Biobank participants from the HGI $(n=2,415$ cases, $n=477,741$ controls) and the participants with the broad respiratory phenotype from 23 andMe ( $n=1,128$ cases, $n=679,531$ controls). This revealed one additional (unreplicated) locus in $C C H C R 1$ at genome-wide significance (using a more-stringent threshold of $P<10^{-8}$ because of the absence of replication opportunities for the meta-analysis) (Table 2).

\section{Mendelian randomization}

Mendelian randomization provides evidence for a causal relationship between an exposure variable and an outcome, given a set of well-characterized assumptions ${ }^{18}$. We used two-sample summary-data Mendelian randomization to assess the evidence in support of causal effects of RNA expression (Genotype-Tissue expression (GTEx) v.7, whole blood) of various genes on the odds of patients becoming critically ill due to COVID-19.

We specified an a priori list of target genes that relate to the mechanism of action of many host-targeted drugs that have been proposed for 


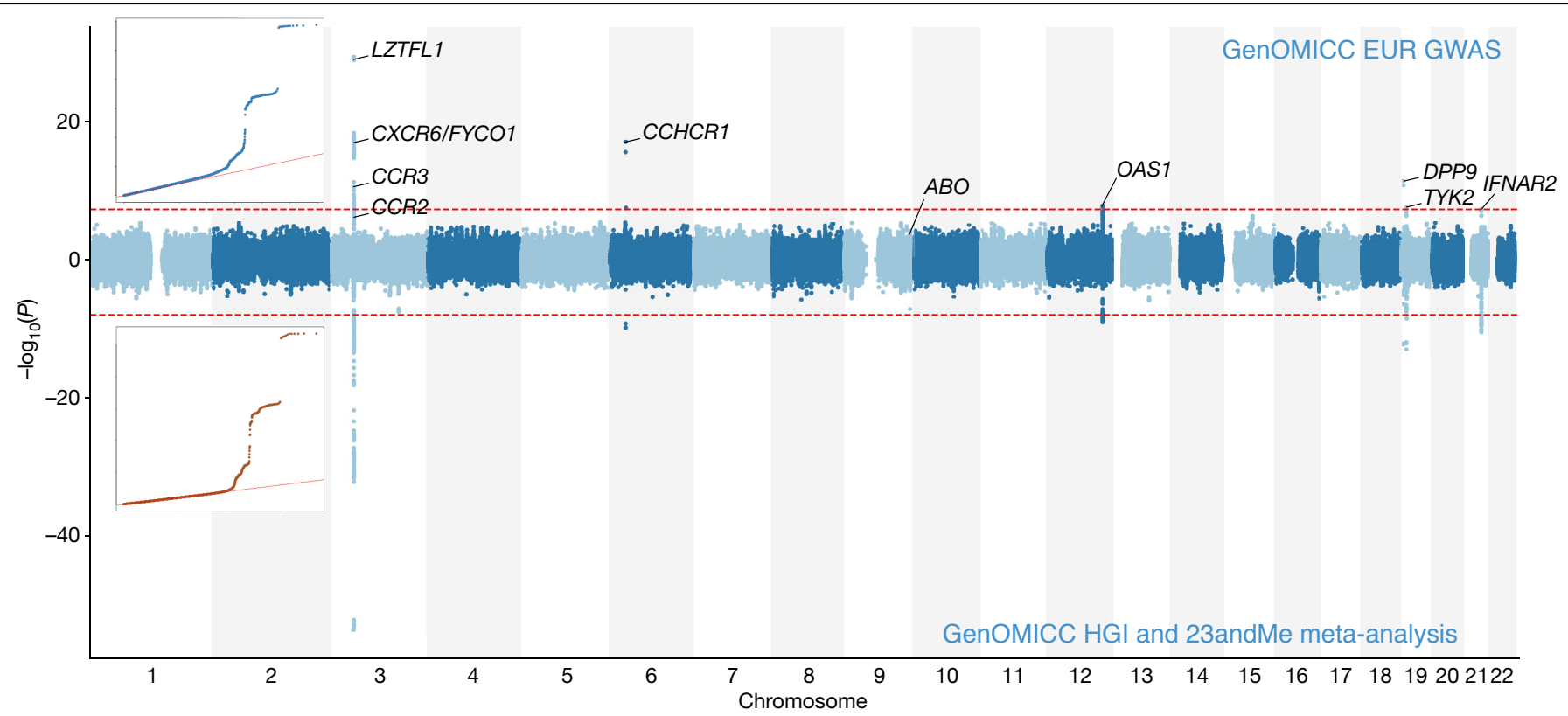

Fig. 1 | Discovery GWAS and meta-analysis results. Miami plot showing the $P$ values for the GenOMICC GWAS in individuals of European ancestry after validation (top) and meta-analysis including patients from the COVID-19 HGI and 23andMe (bottom). Uncorrected $P$ values from GWAS analysis are shown. Top, the red horizontal line indicates genome-wide significance for common variants at $-\log _{10}\left(5 \times 10^{-8}\right)$. Bottom, the red horizontal line indicates a more-stringent genome-wide significance threshold for meta-analysis variants at $-\log _{10}\left(1 \times 10^{-8}\right)$. Insets, quantile-quantile $(Q Q)$ plots of observed versus expected $P$ values that show genomic inflation $(\lambda)$ for each analysis: GenOMICC European cohort, $\lambda=1$.099; GenOMICC HGI-23andMe meta-analysis, $\lambda=1.017$. Full $Q Q$ plots are shown in Extended Data Fig. 1. the treatment of patients with COVID-19(Supplementary Table3). Seven of these targets had a suitable locally acting expression quantitative trait locus (eQTL) in GTEx (v.7). Of these, IFNAR2 remained significant after Bonferroni correction for multiple testing for seven tests $(\beta=-1.49$, s.e. $=0.52, P=0.0043)$ (Supplementary Table 4$)$. There was equivocal evidence of heterogeneity ( $\left.\mathrm{HEIDI}^{19} P=0.015\right)$, indicating that the effect of this variant on critical illness in COVID-19 may be mediated through another mechanism, which may lead to an under- or overestimation of the effect of IFNAR2 expression on the risk of critical illness in COVID-19.

We next performed transcriptome-wide Mendelian randomization to quantify support for unselected genes as potential therapeutic targets. Instruments (genetic variants that affect gene expression) were available for 4,614 unique Ensembl gene IDs. No genes were statistically significant after correcting for multiple comparisons in this analysis $(4,614$ tests). After conservative filtering for heterogeneity (HEIDI $P>0.05$ ), the smallest Mendelian randomization $P=0.00049$ was associated with a variant on chromosome 19:10,466,123 that affects expression of TYK2. Nine other genes with nominally significant Mendelian randomization $P$ values $(P<0.0051)$ were also taken forward for further analysis (Supplementary Table 5).

To replicate these findings, we tested for external evidence using a separate eQTL data set (eQTLgen) ${ }^{20}$ and GWAS (HGI B2, excluding UK Biobank participants). Mendelian randomization signals with consistent directions of effect were significant for IFNAR2 $\left(P=7.5 \times 10^{-4}\right)$ and TYK2 $\left(P=5.5 \times 10^{-5}\right)($ Supplementary Table 6$)$.

\section{Transcriptome-wide association study}

We performed a transcriptome-wide association study (TWAS) ${ }^{21,22}$ to link GWAS results to tissue-specific gene expression data by inferring gene expression from known genetic variants that are associated with transcript abundance (eQTL). For this analysis, we used GTEx v.8 data for two disease-relevant tissues chosen a priori: whole blood and lung samples (Fig. 2). We selected genes with $P<0.05$ in these tissues and performed a combined meta-TWAS analysis ${ }^{23}$, incorporating eQTL data from other tissues in GTEx, to optimize power to detect differences in predicted expression in lung or blood.

We discovered five genes with genome-wide significant differences in predicted expression compared to control individuals (Supplementary Table 7). This included four genes with differential predicted expression in lung tissue (three on chromosome 3, CCR2, CCR3 and CXCR6; and one on chromosome 5, MTA2B) (Supplementary Tables 8-10).

We used meta-analysis by information content (MAIC) ${ }^{24}$ to put these results in the context of existing biological knowledge about host-virus interactions associated with COVID-19. We combined the top 2,000 genes in metaTWAS with previous systematically compiled experimental evidence implicating human genes in SARS-CoV-2 replication and host response. MAIC derives a data-driven weighting for each gene from a range of experimental data sources in the form of gene lists, and outperforms other approaches to providing a composite of multiple lists $^{24}$. We found that the GenOMICC TWAS results had greater overlap with results from transcriptomic, proteomic and CRISPR studies of host genes implicated in COVID-19 than any other data source(Extended Data Fig. 2).

\section{Genetic correlations}

We used a high-definition likelihood method ${ }^{25}$ to provide an initial estimate of heritability based on single-nucleotide polymorphisms (SNPs) (that is, the proportion of phenotypic variance captured by additive effects at common SNPs) for severe COVID-19, which was found to be 0.065 (s.e. $=0.019$ ). We were not able to detect a significant signal for heritability in two additional analyses: first, using controls from the 100,000 Genomes Project (in which matching to the cases from GenOMICC is less close, which may limit heritability estimation) and second, in a smaller GWAS in which some cases from GenOMICC were compared with controls from the UK Biobank, using matched data for body-mass index and age where possible. This second analysis was less powerful because of the lack of close matches for many cases ( $n=1,260$ cases, $n=6300$ controls) (Supplementary Fig. 14). Including 
Table 2 | Meta-analysis of overlapping SNPs between GenOMICC, HGI and 23andMe studies

\begin{tabular}{|c|c|c|c|c|c|c|c|c|c|c|}
\hline SNP & Chr.: pos. & Risk & Alt. & OR $_{\text {gcc }}$ & $\mathrm{Cl}_{\mathrm{gcc}}$ & $P_{\text {gcc }}$ & OR $_{\text {meta }}$ & $\mathbf{C l}_{\text {meta }}$ & $\boldsymbol{P}_{\text {meta }}$ & Locus \\
\hline rs71325088 & 3: $45,862,952$ & C & $T$ & 2.1 & $1.87-2.43$ & $9.3 \times 10^{-30}$ & 1.9 & $1.73-2$ & $2.5 \times 10^{-54}$ & LZTFL1 \\
\hline rs143334143 & $6: 31,121,426$ & $A$ & G & 1.8 & $1.61-2.13$ & $8.8 \times 10^{-18}$ & 1.3 & $1.27-1.48$ & $1.5 \times 10^{-10}$ & $\mathrm{CCHCR} 1$ \\
\hline rs6489867 & $12: 113,363,550$ & $\mathrm{~T}$ & $\mathrm{C}$ & 1.3 & $1.15-1.37$ & $6.9 \times 10^{-7}$ & 1.2 & $1.14-1.25$ & $9.7 \times 10^{-10}$ & OAS1-OAS3 \\
\hline rs2109069 & 19: 4,719,443 & A & $G$ & 1.4 & $1.25-1.48$ & $4 \times 10^{-12}$ & 1.2 & $1.19-1.31$ & $7 \times 10^{-13}$ & DPP9 \\
\hline rs11085727 & 19: 10,466,123 & $\mathrm{T}$ & C & 1.3 & $1.17-1.4$ & $1.3 \times 10^{-7}$ & 1.2 & $1.18-1.31$ & $1.2 \times 10^{-13}$ & $T Y K 2$ \\
\hline rs13050728 & $21: 34,615,210$ & $\mathrm{~T}$ & C & 1.3 & $1.15-1.38$ & $3 \times 10^{-7}$ & 1.2 & $1.16-1.28$ & $5.1 \times 10^{-12}$ & IFNAR2 \\
\hline
\end{tabular}

gcc, GenoMICC European study compared with UK Biobank; meta, combined meta-analysis of all three studies for individuals of European ancestry: GenOMICC ( $n=1,676$ cases, $n=8,380$ controls), $\mathrm{HGI}$ ( $n=2,415$ cases, $n=477,741$ controls) and 23 andMe( $n=1,128$ cases, $n=679,531$ controls).

rare variants in future analyses, which have larger numbers of cases of COVID-19, will provide a more comprehensive estimate of heritability. We also tested for genetic correlations with other traits-that is, the degree to which the underlying genetic components are shared with severe COVID-19. Using the high-definition likelihood method, we identified significant negative genetic correlations with educational attainment and intelligence. Significant positive genetic correlations were detected for a number of adiposity phenotypes including body-mass index and leg fat (Supplementary Fig. 19).

Consistent with GWAS results from other infectious and inflammatory diseases, there was a significant enrichment of strongly associated variants in promoters and enhancers ${ }^{26}$, particularly those identified by the EXaC study as under strong evolutionary selection ${ }^{27}$ (Supplementary Fig. 18). The strongest tissue-type enrichment was in spleen (which may reflect enrichment in immune cells), followed by pancreas (Supplementary Fig. 20).

\section{Discussion}

We have discovered and replicated significant genetic associations that are associated with life-threatening COVID-19 (Fig.1). Our focus on critical illness increases the probability that some of these associations relate to the later, immune-mediated phase of COVID-19 associated with respiratory failure that requires invasive mechanical ventilation ${ }^{2}$. Notably, the GWAS approach is unbiased and genome-wide, enabling the discovery of previously undescribed pathophysiological mechanisms. Because genetic variation can be used to draw a causal inference, genetic evidence in support of a therapeutic target substantially improves the probability of successful drug development ${ }^{28}$. In particular, Mendelian randomization occupies a unique position in the hierarchy of clinical evidence $^{29}$.

Patients admitted to intensive care units in the UK during the first wave of COVID-19 were, on average, younger and less burdened by comorbid illnesses than the hospitalized population ${ }^{14}$. The population studied here was defined by their propensity to critical respiratory failure due to COVID-19. GenOMICC recruited in 208 intensive care units (covering $>95 \%$ of the capacity of intensive care units in the UK), ensuring that a broad spread across the genetic ancestry of patients from the UK was included (Extended Data Fig. 3).

For external replication, the nearest comparison is the analysis of hospitalized patients versus population controls in the COVID-19 HGI, and the broad respiratory phenotype dataset from 23 andMe, which have been generously shared with the international community. We have also immediately made full summary statistics from GenOMICC data openly available at https://genomicc.org/data/.

Despite the differences in case definitions, new associations from our study of critical illness replicate robustly in combined data from studies of hospitalized cases of COVID-19 (Extended Data Table2). Separately, the Mendelian randomization results that suggest a causal role for IFNAR2 and $T Y K 2$ are also statistically significant in confirmatory analyses. Our findings reveal that critical illness in COVID-19 is related to at least two biological mechanisms: innate antiviral defences, which are known to be important early in disease (IFNAR2 and OAS genes), and host-driven inflammatory lung injury, which is a key mechanism of late, life-threatening COVID-19 (DPP9, TYK2 and CCR2) ${ }^{2}$.

Interferons are canonical mediators of antiviral signalling in the host and stimulate release of many essential components of the early host response to viral infection ${ }^{30}$. Consistent with a beneficial role for type I interferons, increased expression of the interferon receptor subunit IFNAR2 reduced the odds of severe COVID-19 with Mendelian randomization discovery $P=0.0043$ (seven tests); replication $P=7.5 \times 10^{-4}$ (one test). Within the assumptions of Mendelian randomization, this represents evidence for a protective role of IFNAR2 in COVID-19. Rare loss-of-function mutations in IFNAR2 are associated with severe COVID-19 ${ }^{12}$ and many other viral disease ${ }^{31,32}$. This suggests that administration of interferon may reduce the probability of critical illness in COVID-19, but our evidence cannot distinguish when during disease progression of COVID-19 such a treatment may be effective. Exogenous interferon treatment did not reduce mortality in hospitalized patients in a large-scale clinical trial ${ }^{33}$, suggesting that this genetic effect may be mediated during the early phase of disease when the viral load is high.

The variant rs10735079 (chromosome $12, P=1.65 \times 10^{-8}$ ) lies in the interferon-inducible oligoadenylate synthetase (OAS) gene cluster (OAS1, OAS2 and OAS3) (Fig. 1). Our TWAS detected significant associations with predicted expression of $O A S 3$ (Fig. 2). OAS1 variants were implicated in susceptibility to SARS-CoV in candidate gene association studies in Vietnam ${ }^{34}$ and China ${ }^{35}$. These genes encode enzymes that produce a host antiviral mediator $\left(2^{\prime}, 5^{\prime}\right.$-oligoadenylate (2-5A)) that activates an effector enzyme, RNase L. RNase L degrades double-stranded $\mathrm{RNA}^{36}$, a replication intermediate of coronaviruses ${ }^{37}$. The betacoronaviruses OC43 and MHV make viral phosphodiesterases that cleave $2-5 \mathrm{~A}^{38}$, but SARS-CoV-2 is not known to have this ability. The OAS genes therefore also provide a potential therapeutic target: endogenous phosphodiesterase 12 (PDE-12) activity degrades 2-5A. Therapeutic PDE-12 inhibitors are available and augment OAS-mediated antiviral activity $^{39}$.

The association on chromosome 19p13.3 ( $\mathrm{rs} 2109069, P=3.98 \times 10^{-12}$ ) is an intronic variant in the gene that encodes dipeptidyl peptidase 9 (DPP9). Variants in this locus are associated with idiopathic pulmonary fibrosis $^{40}$. DPP9 encodes a serine protease that has diverse intracellular functions, including the cleavage of the key antiviral signalling mediator $\mathrm{CXCL10}^{41}$, and key roles in antigen presentation ${ }^{42}$ and inflammosome activation ${ }^{43}$.

As opportunities for therapeutic intervention, particularly experimental therapy, are more abundant in later, more-severe cases of disease, it is important that our results also reveal genes that may act to drive inflammatory organ injury. TYK2 is one of four gene targets for JAK inhibitors such as baricitinib ${ }^{44}$, one of the nine candidate drugs that we used in the creation of our a priori target list (Supplementary Table 3). The association between $T Y K 2$ expression and critical illness was also confirmed in an external dataset (Table 2). 


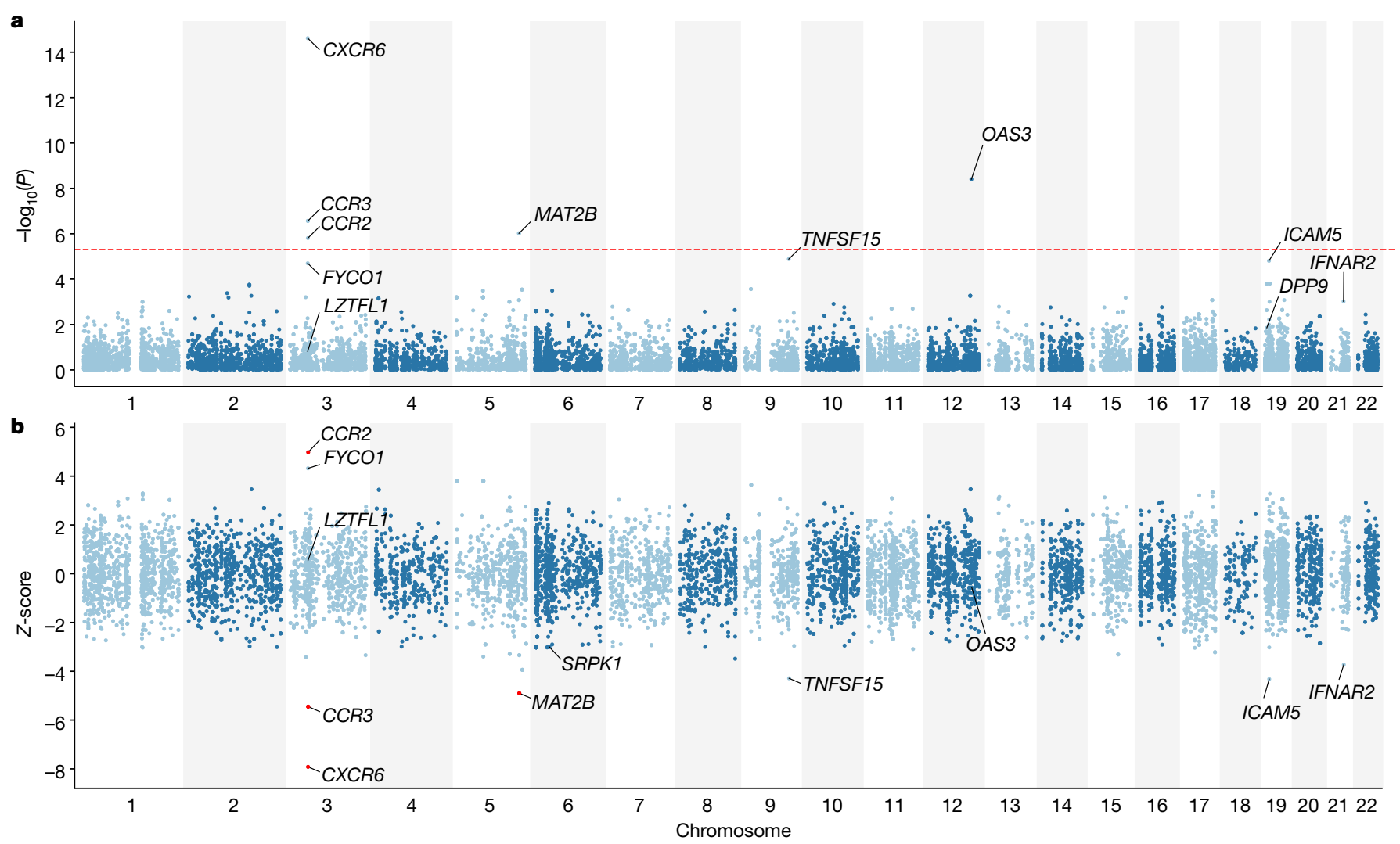

Fig. 2 |Summary of TWAS results. a, Gene-level Manhattan plot showing raw $P$-value results from meta-TWAS analysis across tissues (see Methods). The red horizontal line shows gene-level genome-wide significance at $-\log _{10}\left(5 \times 10^{-6}\right)$. b, $Z$-scores showing the direction of effect for the genotype-inferred expression of transcripts that encode protein-coding genes in lung tissue (GTEx v.8). Red circles indicate genes with genome-wide significance at $P<5 \times 10^{-6}$.
We replicate the association at chromosome $3 \mathrm{p} 21.31$ that has previously been described ${ }^{8}$. The extremely small $P$ value at this locus $\left(P=4.77 \times 10^{-30}\right)$ may reflect the large size of our study, and our focus on extreme severity, as we see a larger effect size in the GenOMICC dataset than in the replication studies (Extended Data Fig. 4). A number of genes in this locus could plausibly explain an association. Our systematic review and meta-analysis of experimental data on infections with betacoronaviruses from other sources provides moderate biological support for $F Y C O 1$, although this additional information comes mostly from in vitro model systems ${ }^{45}$. Our TWAS results show that variants in this region confer genome-wide significant differences in predicted expression of $C X C R 6, C C R 2$ and $C C R 3$ (Fig. 2a); it is likely that at least one of these genes is an important mediator of critical illness in patients with COVID-19.

Association with critical illness for genotype-inferred CCR2 (CC-chemokine receptor 2 ) expression is particularly strong in lung tissue (Fig. 2b). CCR2 promotes chemotaxis of monocytes and macrophages towards sites of inflammation, and there is increased expression of the canonical ligand for CCR2 (monocyte chemoattractant protein (MCP-1)), in bronchoalveolar lavage fluid from the lungs of patients with COVID-19 during mechanical ventilation ${ }^{46}$. Concentrations of circulating MCP-1 are associated with more-severe disease ${ }^{47}$. Anti-CCR2 monoclonal antibody therapy for the treatment of rheumatoid arthritis is safe ${ }^{48}$.

The $A B O$ locus was also previously associated with COVID $-19^{8}$, but did not reach genome-wide significance in the GenOMICC cohort of critically ill patients with COVID-19. Notably, there is a signal close to genome-wide significance at this locus in the combined meta-analysis (Fig.1), suggesting that this variant may be associated with susceptibility to COVID-19, but not critical illness (Extended Data Fig. 4).

Analysis of shared heritability highlights a positive correlation with adiposity. This does not confirm a causal relationship, as a number of biases may affect this analysis. Two effects are likely to be present: first, increased body-mass index and lower socio-economic status are strong risk factors for severe COVID-19 ${ }^{14}$, and second, UK Biobank participants are disproportionately drawn from social groups in which obesity is underrepresented compared with the general population ${ }^{49}$.

Because of the urgency of completing and reporting this study, we have included controls from population genetic studies with systematic differences in population structure, demographics and comorbid diseases, who were genotyped using different technologies compared with the cases that we report. Residual confounding is reflected in the genomic inflation $\left(\lambda_{0.5}\right)$ value of 1.099 for the primary analysis (Extended Data Fig.1). We mitigated the consequent risk of false-positive associations driven by genotyping errors by genotyping the majority of our participants using two different methods (microarray and whole-genome sequencing analyses), and by verifying significant associations using two additional control groups (the 100,000 Genomes Project and Generation Scotland). The success of these mitigations is demonstrated by robust replication of our sentinel SNPs in external studies. Our meta-analysis, combining GenOMICC with multiple additional sources of genome-wide associations, has a $\lambda_{0.5}=1.017$ (Extended Data Fig. 1).

There is an urgent need to extend these findings through further studies. Our MAIC results show that highly ranked genes in the GenOMICC dataset are more likely to be implicated in COVID-19 in other studies (Extended Data Fig. 2). We continue to recruit to the GenOMICC study, in the expectation that additional associations exist and can be detected with larger numbers of cases of COVID-19. Future studies using whole-genome sequencing will search the rarer end of the allele frequency spectrum for variants that increase susceptibility to COVID19. Effect sizes are likely to be greater in the GenOMICC study because the cohort is strongly enriched for immediately life-threatening disease in patients who are either receiving invasive mechanical ventilation, 
or considered by the treating physicians to be at high risk of requiring mechanical support.

We have discovered new and highly plausible genetic associations with critical illness in COVID-19. Some of these associations lead directly to potential therapeutic approaches to augment interferon signalling, antagonize monocyte activation and infiltration into the lungs, or specifically target harmful inflammatory pathways. Although this adds substantially to the biological rationale that underpins specific therapeutic approaches, each treatment must be tested in large-scale clinical trials before entering clinical practice.

\section{Online content}

Any methods, additional references, Nature Research reporting summaries, source data, extended data, supplementary information, acknowledgements, peer review information; details of author contributions and competing interests; and statements of data and code availability are available at https://doi.org/10.1038/s41586-020-03065-y.

1. Dorward, D. A. et al. Tissue-specific immunopathology in fatal COVID-19. Am. J. Respir. Crit. Care Med. https://doi.org/10.1164/rccm.202008-3265OC (2020).

2. The RECOVERY Collaborative Group. Dexamethasone in hospitalized patients with COVID-19 - preliminary report. N. Engl. J. Med. https://doi.org/10.1056/NEJMoa2021436 (2020).

3. Baillie, J. K. Targeting the host immune response to fight infection. Science $\mathbf{3 4 4}, 807-808$ (2014).

4. Sørensen, T. I., Nielsen, G. G., Andersen, P. K. \& Teasdale, T. W. Genetic and environmental influences on premature death in adult adoptees. N. Engl. J. Med. 318, 727-732 (1988).

5. Patarčić, I. et al. The role of host genetic factors in respiratory tract infectious diseases: systematic review, meta-analyses and field synopsis. Sci. Rep. 5, 16119 (2015).

6. Horby, P., Nguyen, N. Y., Dunstan, S. J. \& Baillie, J. K. An updated systematic review of the role of host genetics in susceptibility to influenza. Influenza Other Respir. Viruses 7 (Suppl 2), 37-41 (2013).

7. Clohisey, S. \& Baillie, J. K. Host susceptibility to severe influenza A virus infection. Crit. Care 23, 303 (2019).

8. The Severe Covid-19 GWAS Group. Genomewide association study of severe COVID-19 with respiratory failure. N. Engl. J. Med. 383, 1522-1534 (2020).

9. Shelton, J. F. et al. Trans-ethnic analysis reveals genetic and non-genetic associations with COVID-19 susceptibility and severity. Preprint at https://doi.org/10.1101/2020.09.04. 20188318 (2020).

10. Casanova, J.-L. Severe infectious diseases of childhood as monogenic inborn errors of immunity. Proc. Natl Acad. Sci. USA 112, E7128-E7137 (2015).

11. Plenge, R. M. Molecular underpinnings of severe coronavirus disease 2019. J. Am. Med. Assoc. 324, 638-639 (2020).

12. Zhang, Q. et al. Inborn errors of type I IFN immunity in patients with life-threatening COVID-19. Science 370, eabd4570 (2020).

13. Millar, J. E. et al. Robust, reproducible clinical patterns in hospitalised patients with COVID-19. Preprint at https://doi.org/10.1101/2020.08.14.20168088 (2020).

14. Docherty, A. B. et al. Features of 20133 UK patients in hospital with COVID-19 using the ISARIC WHO Clinical Characterisation Protocol: prospective observational cohort study. Br. Med. J. 369, m1985 (2020).

15. Angus, D. C. et al. Effect of hydrocortisone on mortality and organ support in patients with severe COVID-19: the remap-cap COVID-19 corticosteroid domain randomized clinical trial. J. Am. Med. Assoc. 324, 1317-1329 (2020).

16. Carvelli, J. et al. Association of COVID-19 inflammation with activation of the C5a-C5aR1 axis. Nature 588, 146-150 (2020).

17. Baillie, J. K. \& Digard, P. Influenza - time to target the host? N. Engl. J. Med. 369, 191-193 (2013).

18. Bretherick, A. D. et al. Linking protein to phenotype with Mendelian randomization detects 38 proteins with causal roles in human diseases and traits. PLoS Genet. 16, e1008785 (2020).

19. Zhu, Z. et al. Integration of summary data from GWAS and eQTL studies predicts complex trait gene targets. Nat. Genet. 48, 481-487 (2016).

20. Võsa, U. et al. Unraveling the polygenic architecture of complex traits using blood eQTL metaanalysis. Preprint at https://doi.org/10.1101/447367 (2018).

21. Gamazon, E. R. et al. A gene-based association method for mapping traits using reference transcriptome data. Nat. Genet. 47, 1091-1098 (2015).

22. Gusev, A. et al. Integrative approaches for large-scale transcriptome-wide association studies. Nat. Genet. 48, 245-252 (2016).

23. Barbeira, A. N. et al. Exploring the phenotypic consequences of tissue specific gene expression variation inferred from GWAS summary statistics. Nat. Commun. 9, 1825 (2018).

24. Li, B. et al. Genome-wide CRISPR screen identifies host dependency factors for influenza A virus infection. Nat. Commun. 11, 164 (2020).

25. Ning, Z., Pawitan, Y. \& Shen, X. High-definition likelihood inference of genetic correlations across human complex traits. Nat. Genet. 52, 859-864 (2020).

26. The FANTOM Consortium and the RIKEN PMI and CLST (DGT). A promoter-level mammalian expression atlas. Nature 507, 462-470 (2014).

27. Villar, D. et al. Enhancer evolution across 20 mammalian species. Cell 160, 554-566 (2015).
28. Plenge, R. M., Scolnick, E. M. \& Altshuler, D. Validating therapeutic targets through human genetics. Nat. Rev. Drug Discov. 12, 581-594 (2013).

29. Davies, N. M., Holmes, M. V. \& Davey Smith, G. Reading Mendelian randomisation studies: a guide, glossary, and checklist for clinicians. Br. Med. J. 362, k601 (2018).

30. Sadler, A. J. \& Williams, B. R. G. Interferon-inducible antiviral effectors. Nat. Rev. Immunol. 8, 559-568 (2008)

31. Hambleton, S. et al. STAT2 deficiency and susceptibility to viral illness in humans. Proc. Natl Acad. Sci. USA 110, 3053-3058 (2013).

32. Duncan, C. J. A. et al. Human IFNAR2 deficiency: lessons for antiviral immunity. Sci. Transl. Med. 7, 307ra154 (2015).

33. WHO Solidarity Trial Consortium. Repurposed antiviral drugs for COVID-19-interim WHO Solidarity trial results. N. Engl. J. Med. 384, 497-511 (2020).

34. Hamano, E. et al. Polymorphisms of interferon-inducible genes OAS-1 and MxA associated with SARS in the Vietnamese population. Biochem. Biophys. Res. Commun. 329, 1234-1239 (2005).

35. He, J. et al. Association of SARS susceptibility with single nucleic acid polymorphisms of OAS1 and MXA genes: a case-control study. BMC Infect. Dis. 6, 106 (2006).

36. Choi, U. Y., Kang, J.-S., Hwang, Y. S. \& Kim, Y.-J. Oligoadenylate synthase-like (OASL) proteins: dual functions and associations with diseases. Exp. Mol. Med. 47, e144 (2015).

37. Hagemeijer, M. C., Vonk, A. M., Monastyrska, I., Rottier, P. J. M. \& de Haan, C. A. Visualizing coronavirus RNA synthesis in time by using click chemistry. J. Virol. 86, 5808-5816 (2012)

38. Silverman, R. H. \& Weiss, S. R. Viral phosphodiesterases that antagonize double-stranded RNA signaling to RNase L by degrading 2-5A. J. Interferon Cytokine Res. 34, 455-463 (2014).

39. Wood, E. R. et al. The role of phosphodiesterase 12 (PDE12) as a negative regulator of the innate immune response and the discovery of antiviral inhibitors. J. Biol. Chem. 290, 19681-19696 (2015).

40. Fingerlin, T. E. et al. Genome-wide association study identifies multiple susceptibility loci for pulmonary fibrosis. Nat. Genet. 45, 613-620 (2013).

41. Zhang, $\mathrm{H}$. et al. Identification of novel dipeptidyl peptidase 9 substrates by two-dimensional differential in-gel electrophoresis. FEBS J. 282, 3737-3757 (2015).

42. Geiss-Friedlander, R. et al. The cytoplasmic peptidase DPP9 is rate-limiting for degradation of proline-containing peptides. J. Biol. Chem. 284, 27211-27219 (2009)

43. Griswold, A. R. et al. DPP9's enzymatic activity and not its binding to CARD8 inhibits inflammasome activation. ACS Chem. Biol. 14, 2424-2429 (2019).

44. Nguyen, D.-T. et al. Pharos: collating protein information to shed light on the druggable genome. Nucleic Acids Res. 45 (D1), D995-D1002 (2017).

45. Parkinson, N. et al. Systematic review and meta-analysis identifies potential host therapeutic targets in COVID-19. Preprint at https://doi.org/10.1101/2020.08.27.20182238 (2020).

46. Zhou, Z. et al. Heightened innate immune responses in the respiratory tract of COVID-19 patients. Cell Host Microbe 27, 883-890 (2020).

47. Zhao, Y. et al. Longitudinal COVID-19 profiling associates IL-1RA and IL-10 with disease severity and RANTES with mild disease. JCI Insight 5, e139834 (2020).

48. Vergunst, C. E. et al. Modulation of CCR2 in rheumatoid arthritis: a double-blind, randomized, placebo-controlled clinical trial. Arthritis Rheum. 58, 1931-1939 (2008).

49. Sudlow, C. et al. UK Biobank: an open access resource for identifying the causes of a wide range of complex diseases of middle and old age. PLoS Med. 12, e1001779 (2015).

Publisher's note Springer Nature remains neutral with regard to jurisdictional claims in published maps and institutional affiliations.

(c) The Author(s), under exclusive licence to Springer Nature Limited 2020

Erola Pairo-Castineira ${ }^{1,2,329}$, Sara Clohisey ${ }^{1,329}$, Lucija Klaric ${ }^{2,329}$, Andrew D. Bretherick ${ }^{2,329}$, Konrad Rawlik, ${ }^{1,329}$, Dorota Pasko ${ }^{3}$, Susan Walker ${ }^{3}$, Nick Parkinson', Max Head Fourman', Clark D. Russell ${ }^{1,4}$, James Furniss', Anne Richmond ${ }^{2}$, Elvina Gountouna ${ }^{5}$, Nicola Wrobel ${ }^{6}$, David Harrison ${ }^{7}$, Bo Wang', Yang Wu ${ }^{8}$, Alison Meynert ${ }^{2}$, Fiona Griffiths', Wilna Oosthuyzen', Athanasios Kousathanas ${ }^{3}$, Loukas Moutsianas ${ }^{3}$, Zhijian Yang ${ }^{9}$, Ranran Zhai ${ }^{9}$, Chenqing Zheng ${ }^{9}$, Graeme Grimes ${ }^{2}$, Rupert Beale ${ }^{10}$, Jonathan Millar', Barbara Shih', Sean Keating ${ }^{11}$, Marie Zechner', Chris Haley', David J. Porteous ${ }^{5}$, Caroline Hayward ${ }^{2,5}$, Jian Yang ${ }^{12,13}$, Julian Knight ${ }^{14}$, Charlotte Summers ${ }^{15}$, Manu Shankar-Hari ${ }^{16,17}$,

Paul Klenerman $^{14}$, Lance Turtle ${ }^{18}$, Antonia Ho ${ }^{19}$, Shona C. Moore ${ }^{18}$, Charles Hinds ${ }^{20}$, Peter Horby ${ }^{21}$, Alistair Nichol ${ }^{22,23,24}$, David Maslove ${ }^{25}$, Lowell Ling ${ }^{26}$, Danny McAuley ${ }^{27,28}$ Hugh Montgomery ${ }^{29}$, Timothy Walsh ${ }^{11}$, Alexandre C. Pereira ${ }^{30,31}$, Alessandra Renieri ${ }^{32,33}$, The GenOMICC Investigators*, The ISARIC4C Investigators*,

The COVID-19 Human Genetics Initiative* ${ }^{*}$ 23andMe Investigators*, BRACOVID Investigators*, Gen-COVID Investigators* ${ }^{*}$ Xia Shen ${ }^{9,34,35}$, Chris P. Ponting ${ }^{2}$, Angie Fawkes ${ }^{6}$, Albert Tenesa ${ }^{1,2,34}$, Mark Caulfield ${ }^{3,20}$, Richard Scott ${ }^{3,36}$, Kathy Rowan $^{7}$, Lee Murphy ${ }^{6}$, Peter J. M. Openshaw ${ }^{37,38}$, Malcolm G. Semple ${ }^{18,39}$, Andrew Law', Veronique Vitart ${ }^{2}$, James F. Wilson ${ }^{2,34}$ \& J. Kenneth Baillie ${ }^{1,2,11 \bowtie}$

${ }^{1}$ Roslin Institute, University of Edinburgh, Edinburgh, UK. ${ }^{2} \mathrm{MRC}$ Human Genetics Unit, Institute of Genetics and Molecular Medicine, University of Edinburgh, Western General Hospital, Edinburgh, UK. ${ }^{3}$ Genomics England, London, UK. ${ }^{4}$ Centre for Inflammation Research, The Queen's Medical Research Institute, University of Edinburgh, Edinburgh, UK. ${ }^{5}$ Centre for Genomic and Experimental Medicine, Institute of Genetics and Molecular Medicine, University of Edinburgh, Western General Hospital, Edinburgh, UK. ${ }^{6}$ Edinburgh Clinical Research Facility, Western General Hospital, University of Edinburgh, Edinburgh, UK. Intensive Care National Audit \& Research Centre, London, UK. ${ }^{8}$ Institute for Molecular 


\section{Article}

Bioscience, The University of Queensland, Brisbane, Queensland, Australia. ${ }^{9}$ Biostatistics Group, School of Life Sciences, Sun Yat-sen University, Guangzhou, China. ${ }^{10}$ The Crick Institute, London, UK. "Intensive Care Unit, Royal Infirmary of Edinburgh, Edinburgh, UK. ${ }^{12}$ School of Life Sciences, Westlake University, Hangzhou, China. ${ }^{13}$ Westlake Laboratory of Life Sciences and Biomedicine, Hangzhou, China. ${ }^{14}$ Wellcome Centre for Human Genetics, University of Oxford, Oxford, UK. ${ }^{15}$ Department of Medicine, University of Cambridge, Cambridge, UK. ${ }^{16}$ Department of Intensive Care Medicine, Guy's and St Thomas' NHS Foundation Trust, London, UK. ${ }^{17}$ School of Immunology and Microbial Sciences, King's College London, London, UK. ${ }^{18} \mathrm{NIHR}$ Health Protection Research Unit for Emerging and Zoonotic Infections, Institute of Infection, Veterinary and Ecological Sciences, University of Liverpool, Liverpool, UK. ${ }^{19} \mathrm{MRC}$-University of Glasgow Centre for Virus Research, Institute of Infection, Immunity and Inflammation, College of Medical, Veterinary and Life Sciences, University of Glasgow, Glasgow, UK. ${ }^{20}$ William Harvey Research Institute, Barts and the London School of Medicine and Dentistry, Queen Mary University of London, London, UK. ${ }^{21}$ Centre for Tropical Medicine and Global Health, Nuffield Department of Medicine, University of Oxford, Oxford, UK. ${ }^{22}$ Clinical Research Centre at St Vincent's University Hospital, University College Dublin, Dublin, Ireland. ${ }^{23}$ Australian and New Zealand Intensive Care Research Centre, Monash University, Melbourne, Victoria, Australia. ${ }^{24}$ Intensive Care Unit, Alfred Hospital, Melbourne, Victoria, Australia. ${ }^{25}$ Department of Critical Care Medicine,
Queen's University and Kingston Health Sciences Centre, Kingston, Ontario, Canada. ${ }^{26}$ Department of Anaesthesia and Intensive Care, The Chinese University of Hong Kong, Prince of Wales Hospital, Hong Kong, China. ${ }^{27}$ Wellcome-Wolfson Institute for Experimental Medicine, Queen's University Belfast, Belfast, UK. ${ }^{28}$ Department of Intensive Care Medicine, Royal Victoria Hospital, Belfast, UK. ${ }^{29}$ UCL Centre for Human Health and Performance, University College London, London, UK. ${ }^{30}$ Faculty of Medicine, University of São Paulo, São Paulo, Brazil. ${ }^{31} \mathrm{Heart}$ Institute, University of São Paulo, São Paulo, Brazil. ${ }^{32}$ Medical Genetics, University of Siena, Siena, Italy. ${ }^{33}$ Genetica Medica, Azienda Ospedaliero-Universitaria Senese, Siena, Italy. ${ }^{34}$ Centre for Global Health Research, Usher Institute of Population Health Sciences and Informatics, Edinburgh, UK. ${ }^{35}$ Department of Medical Epidemiology and Biostatistics, Karolinska Institutet, Stockholm, Sweden. ${ }^{36}$ Great Ormond Street Hospital for Children NHS Foundation Trust, London, UK. ${ }^{37}$ National Heart and Lung Institute, Imperial College London, London, UK. ${ }^{38}$ Imperial College Healthcare NHS Trust London, London, UK. ${ }^{39}$ Respiratory Medicine, Alder Hey Children's Hospital, Institute in The Park University of Liverpool, Liverpool, UK. ${ }^{329}$ These authors contributed equally: Erola Pairo-Castineira, Sara Clohisey, Lucija Klaric, Andrew D. Bretherick, Konrad Rawlik. * Lists of authors and their affiliations appear in the online version of the paper. A full list of members and their affiliations appears in the Supplementary Information. 凶e-mail: j.k.baillie@ed.ac.uk 


\section{GenOMICC Consortium}

GenOMICC co-investigators

Sara Clohisey ${ }^{1,329}$, Peter Horby ${ }^{21}$, Johnny Millar ${ }^{1}$, Julian Knight ${ }^{14}$, Hugh Montgomery ${ }^{29}$ David Maslove $^{25}$, Lowell Ling ${ }^{26}$, Alistair Nichol ${ }^{22}$, Charlotte Summers ${ }^{15}$, Tim Walsh",

Charles Hinds $^{20}$, Malcolm G. Semple ${ }^{18,39}$, Peter J. M. Openshaw ${ }^{37,38}$, Manu Shankar-Hari' ${ }^{16}$ Antonia $\mathrm{Ho}^{19}$, Danny McAuley ${ }^{27,28}$, Chris Ponting ${ }^{2}$, Kathy Rowan ${ }^{7}$ \& J. Kenneth Baillie ${ }^{1,2,11}$

\section{Central management and laboratory team}

Fiona Griffiths', Wilna Oosthuyzen', Jen Meikle', Paul Finernan', James Furniss' Ellie Mcmaster', Andy Law', Sara Clohisey ${ }^{1,329}$, J. Kenneth Baillie ${ }^{1,11}$, Trevor Paterson' Tony Wackett', Ruth Armstrong ${ }^{1}$, Lee Murphy ${ }^{6}$, Angie Fawkes ${ }^{6}$, Richard Clark ${ }^{6}$ Audrey Coutts ${ }^{6}$, Lorna Donnelly ${ }^{6}$, Tammy Gilchrist ${ }^{6}$, Katarzyna Hafezi ${ }^{6}$,

Louise Macgillivray ${ }^{6}$, Alan Maclean ${ }^{6}$, Sarah McCafferty ${ }^{6}$, Kirstie Morrice ${ }^{6}$, Jane Weaver', Ceilia Boz', Ailsa Golightly', Mari Ward', Hanning Mal', Helen Szoor-McElhinney', Adam Brown', Ross Hendry', Andrew Stenhouse', Louise Cullum', Dawn Law', Sarah Law', Rachel Law', Max Head Fourman', Maaike Swets', Nicky Day', Filip Taneski',

Esther Duncan', Marie Zechner' \& Nicholas Parkinson'

\section{Data analysis team}

Erola Pairo-Castineira ${ }^{1,2,329}$, Sara Clohisey ${ }^{1,329}$, Lucija Klaric ${ }^{2,329}$, Andrew D. Bretherick ${ }^{2,329}$ Konrad Rawlik ${ }^{1,329}$, Dorota Pasko ${ }^{3}$, Susan Walker ${ }^{3}$, Nick Parkinson', Max Head Fourman', Clark D. Russell ${ }^{1,4}$, James Furniss' ${ }^{1}$, Anne Richmond ${ }^{2}$, Elvina Gountouna ${ }^{5}$, David Harrison ${ }^{7}$, Bo Wang', Yang Wu ${ }^{8}$, Alison Meynert ${ }^{2}$, Athanasios Kousathanas ${ }^{3}$, Loukas Moutsianas ${ }^{3}$, Zhijian Yang ${ }^{9}$, Ranran Zhai ${ }^{9}$, Chenqing Zheng ${ }^{9}$, Graeme Grimes ${ }^{2}$, Jonathan Millar', Barbara Shih', Marie Zechner', Jian Yang ${ }^{12,13}$, Xia Shen ${ }^{9,34,35}$, Chris P. Ponting ${ }^{2}$, Albert Tenesa ${ }^{1,2,34}$, Kathy Rowan ${ }^{7}$, Andrew Law', Veronique Vitart ${ }^{2}$, James F. Wilson ${ }^{2,34}$ \& J. Kenneth Baillie ${ }^{1,2,11}$

Barts Health NHS Trust, London, UK

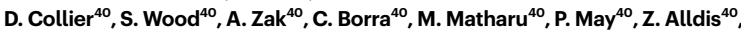

O. Mitchelmore ${ }^{40}$, R. Bowles ${ }^{40}$, A. Easthope ${ }^{40}$, F. Bibi $^{40}$, I. Lancoma-Malcolm $^{40}$

J. Gurasashvili ${ }^{40}$, J. Pheby ${ }^{40}$, J. Shiel ${ }^{40}$, M. Bolton ${ }^{40}$, M. Patel ${ }^{40}$, M. Taylor $^{40}$, O. Zongo ${ }^{40}$,

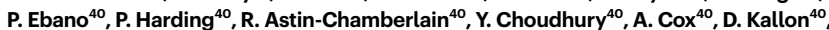

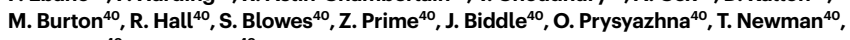

C. Tierney ${ }^{40} \&$ J. Kassam ${ }^{40}$

Guy's \& St Thomas' Hospital, London, UK

M. Shankar-Hari ${ }^{41}$, M. Ostermann ${ }^{41}$, S. Campos ${ }^{41}$, A. Bociek ${ }^{41}$, R. Lim ${ }^{41}$, N. Grau ${ }^{41}$, T. O. Jones ${ }^{41}$, C. Whitton ${ }^{41}$, M. Marotti ${ }^{41}$ \& G. Arbane ${ }^{41}$

James Cook University Hospital, Middlesburgh, UK

S. Bonner ${ }^{42}$, K. Hugill ${ }^{42}$ \& J. Reid ${ }^{42}$

The Royal Liverpool University Hospital, Liverpool, UK

I. Welters ${ }^{43}$, V. Waugh ${ }^{43}$, K. Williams ${ }^{43}$, D. Shaw ${ }^{43}$, J. Fernandez Roman ${ }^{43}$

M. Lopez Martinez ${ }^{43}$, E. Johnson ${ }^{43}$, A. Waite ${ }^{43}$, B. Johnston ${ }^{43}$, D. Hamilton ${ }^{43}$ \& S. Mulla ${ }^{43}$

King's College Hospital, London, UK

M. McPhail ${ }^{44} \&$ J. Smith ${ }^{44}$

Royal Infirmary of Edinburgh, Edinburgh, UK

J. K. Baillie ${ }^{1,2,11}$, L. Barclay ${ }^{45}$, D. Hope ${ }^{45}$, C. McCulloch ${ }^{45}$, L. McQuillan ${ }^{45}$, S. Clark ${ }^{45}$

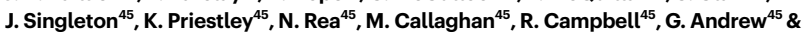

L. Marshall ${ }^{45}$

John Radcliffe Hospital, Oxford, UK

S. McKechnie ${ }^{46}$, P. Hutton ${ }^{46}$, A. Bashyal ${ }^{46}$ \& N. Davidson ${ }^{46}$

Addenbrooke's Hospital, Cambridge, UK

C. Summers ${ }^{47}$, P. Polgarova ${ }^{47}$, K. Stroud ${ }^{47}$, N. Pathan ${ }^{47}$, K. Elston ${ }^{47}$ \& S. Agrawal ${ }^{47}$

Morriston Hospital, Swansea, UK

C. Battle ${ }^{48}$, L. Newey ${ }^{48}$, T. Rees ${ }^{48}$, R. Harford ${ }^{48}$, E. Brinkworth ${ }^{48}$, M. Williams ${ }^{48}$ \& C. Murphy ${ }^{48}$

Ashford \& St Peter's Hospital, Surrey, UK

I. White ${ }^{49}$ \& M. Croft ${ }^{49}$

Royal Stoke University Hospital, Staffordshire, UK

N. Bandla ${ }^{50}$, M. Gellamucho ${ }^{50}$, J. Tomlinson ${ }^{50}$, H. Turner ${ }^{50}$, M. Davies $^{50}$, A. Quinn ${ }^{50}$,

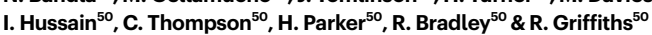

Queen Elizabeth Hospital, Birmingham, UK

J. Scriven ${ }^{51}$, A. Nilsson ${ }^{51}$, M. Bates ${ }^{51}$, J. Dasgin ${ }^{51}$ \& J. Gill ${ }^{51}$

Glasgow Royal Infirmary, Glasgow, UK

A. Puxty ${ }^{52}$, S. Cathcart ${ }^{52}$, D. Salutous ${ }^{52}$, L. Turner ${ }^{52}$, K. Duffy ${ }^{52} \&$ K. Puxty ${ }^{52}$

Kingston Hospital, Surrey, UK

A. Joseph ${ }^{53}$, R. Herdman-Grant ${ }^{53}$, R. Simms ${ }^{53}$, A. Swain ${ }^{53}$, A. Naranjo ${ }^{53}$, R. Crowe ${ }^{53}$,

K. Sollesta ${ }^{53}$, A. Loveridge ${ }^{53}$, D. Baptista ${ }^{53}$ \& E. Morino ${ }^{53}$
The Tunbridge Wells Hospital \& Maidstone Hospital, Kent, UK

M. Davey ${ }^{54}$, D. Golden ${ }^{54}$ \& J. Jones ${ }^{54}$

North Middlesex University Hospital NHS Trust, London, UK

J. Moreno Cuesta ${ }^{55}$, A. Haldeos ${ }^{55}$, D. Bakthavatsalam ${ }^{55}$, R. Vincent ${ }^{55}$, M. Elhassan ${ }^{55}$,

K. Xavier ${ }^{55}$, A. Ganesan ${ }^{55}$, D. Purohit ${ }^{55}$ \& M. Abdelrazik ${ }^{55}$

Bradford Royal Infirmary, Bradford, UK

J. Morgan ${ }^{56}$, L. Akeroyd ${ }^{56}$, S. Bano ${ }^{56}$, T. Lawton ${ }^{56}$, D. Warren ${ }^{56}$, M. Bromley ${ }^{56}$, K. Sellick ${ }^{56}$

L. Gurr ${ }^{56}$, B. Wilkinson ${ }^{56}$, V. Nagarajan ${ }^{56}$ \& P. Szedlak ${ }^{56}$

Blackpool Victoria Hospital, Blackpool, UK

J. Cupitt ${ }^{57}$, E. Stoddard ${ }^{57}$, L. Benham ${ }^{57}$, S. Preston ${ }^{57}$, S. Laha ${ }^{57}$, N. Slawson ${ }^{57}$, Z. Bradshaw ${ }^{57}$,

J. Brown ${ }^{57}$, M. Caswell ${ }^{57}$ \& S. Melling ${ }^{57}$

Countess of Chester Hospital, Chester, UK

P. Bamford ${ }^{58}$, M. Faulkner ${ }^{58}$, K. Cawley ${ }^{58}$, H. Jeffrey ${ }^{58}$, E. London ${ }^{58}$, H. Sainsbury ${ }^{58}$, I. Nagra ${ }^{58}$,

F. Nasir ${ }^{58}$, C. Dunmore ${ }^{58}$, R. Jones ${ }^{58}$, A. Abraheem ${ }^{58}$, M. Al-Moasseb ${ }^{58} \&$ R. Girach ${ }^{58}$

Wythenshawe Hospital, Manchester, UK

G. Padden ${ }^{59}$, J. Egan ${ }^{59}$, C. Brantwood ${ }^{59}$, P. Alexander ${ }^{59}$, J. Bradley-Potts ${ }^{59}$, S. Allen ${ }^{59}$ \& T. Felton ${ }^{59}$

St George's Hospital, London, UK

S. Manna ${ }^{60}$, S. Farnell-Ward ${ }^{60}$, S. Leaver ${ }^{60}$, J. Queiroz $^{60}$, E. Maccacari ${ }^{60}$, D. Dawson ${ }^{60}$,

C. Castro Delgado ${ }^{60}$, R. Pepermans Saluzzio ${ }^{60}$, O. Ezeobu ${ }^{60}$, L. Ding ${ }^{60}$, C. Sicat ${ }^{60}$, R. Kanu ${ }^{60}$

G. Durrant ${ }^{60}$, J. Texeira $^{60}$, A. Harrison ${ }^{60} \&$ T. Samakomva ${ }^{60}$

Good Hope Hospital, Birmingham, UK

J. Scriven ${ }^{61}$, H. Willis ${ }^{61}$, B. Hopkins ${ }^{61}$ \& L. Thrasyvoulou ${ }^{61}$

Stepping Hill Hospital, Stockport, UK

M. Jackson ${ }^{62}$, A. Zaki ${ }^{62}$, C. Tibke ${ }^{62}$, S. Bennett ${ }^{62}$, W. Woodyatt ${ }^{62}$, A. Kent ${ }^{62}$ \& E. Goodwin ${ }^{62}$

Manchester Royal Infirmary, Manchester, UK

C. Brandwood ${ }^{63}$, R. Clark $^{63}$ \& L. Smith ${ }^{63}$

Royal Alexandra Hospital, Paisley, UK

K. Rooney ${ }^{64}$, N. Thomson ${ }^{64}$, N. Rodden ${ }^{64}$, E. Hughes ${ }^{64}$, D. McGlynn ${ }^{64}$, C. Clark ${ }^{64}$, P. Clark ${ }^{64}$

L. Abel ${ }^{64}$, R. Sundaram ${ }^{64}$, L. Gemmell ${ }^{64}$, M. Brett ${ }^{64}$, J. Hornsby ${ }^{64}$, P. MacGoey ${ }^{64}$, R. Price ${ }^{64}$

B. Digby ${ }^{64}$, P. O'Neil ${ }^{64}$, P. McConnell ${ }^{64}$ \& P. Henderson ${ }^{64}$

Queen Elizabeth University Hospital, Glasgow, UK

S. Henderson ${ }^{65}$, M. Sim ${ }^{65}$, S. Kennedy-Hay ${ }^{65}$, C. McParland ${ }^{65}$, L. Rooney ${ }^{65}$ \& N. Baxter ${ }^{65}$

Queen Alexandra Hospital, Portsmouth, UK

D. Pogson ${ }^{66}$, S. Rose ${ }^{66}$, Z. Daly ${ }^{66}$ \& L. Brimfield ${ }^{66}$

BHRUT (Barking Havering) - Queens Hospital \& King George Hospital, Essex, UK M. K. Phull ${ }^{67}$, M. Hussain ${ }^{67}$, T. Pogreban ${ }^{67}$, L. Rosaroso ${ }^{67}$, E. Salciute ${ }^{67}$ \& L. Grauslyte ${ }^{67}$

University College Hospital, London, UK

D. Brealey ${ }^{68}$, E. Raith ${ }^{68}$, N. MacCallum ${ }^{68}$, G. Bercades ${ }^{68}$, I. Hass $^{68}$, D. Smyth $^{68}$, A. Reyes ${ }^{68}$ \& G. Martir ${ }^{68}$

Royal Victoria Infirmary, Newcastle Upon Tyne, UK

I. D. Clement ${ }^{69}$, K. Webster ${ }^{69}$, C. Hays $^{69}$ \& A. Gulati ${ }^{69}$

Western Sussex Hospitals, West Sussex, UK

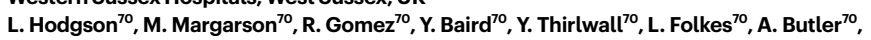

E. Meadows ${ }^{70}$, S. Moore ${ }^{70}$, D. Raynard ${ }^{70}$, H. Fox ${ }^{70}$, L. Riddles ${ }^{70}$, K. King $^{70}$, S. Kimber $^{70}$,

G. Hobden ${ }^{70}$, A. McCarthy ${ }^{70}$, V. Cannons ${ }^{70}$, I. Balagosa ${ }^{70}$, I. Chadbourn ${ }^{70}$ \& A. Gardner ${ }^{70}$

Salford Royal Hospital, Manchester, UK

D. Horner ${ }^{71}$, D. McLaughlanv ${ }^{71}$, B. Charles ${ }^{71}$, N. Proudfoot ${ }^{71}$, T. Marsden ${ }^{71}$, L. McMorrow ${ }^{71}$,

B. Blackledge ${ }^{71}$, J. Pendlebury ${ }^{71}$, A. Harvey ${ }^{71}$, E. Apetri ${ }^{71}$, C. Basikolo ${ }^{71}$, L. Catlow ${ }^{71}$,

R. Doonan ${ }^{71}$, K. Knowles ${ }^{71}$, S. Lee ${ }^{71}$, D. Lomas ${ }^{71}$, C. Lyons ${ }^{71}$, J. Perez ${ }^{71}$, M. Poulaka ${ }^{71}$,

M. Slaughter ${ }^{71}$, K. Slevin ${ }^{71}$, M. Taylor ${ }^{71}$, V. Thomas ${ }^{71}$, D. Walker ${ }^{71}$ \& J. Harris ${ }^{71}$

The Royal Oldham Hospital, Manchester, UK

A. Drummond ${ }^{72}$, R. Tully ${ }^{72}$, J. Dearden ${ }^{72}$, J. Philbin ${ }^{72}$, S. Munt ${ }^{72}$, C. Rishton ${ }^{72}$, G. O'Connor ${ }^{72}$,

M. Mulcahy ${ }^{72}$, E. Dobson ${ }^{72}$, J. Cuttler ${ }^{72} \&$ M. Edward ${ }^{72}$

Pinderfields General Hospital, Wakefield, UK

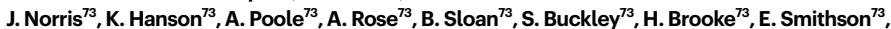

R. Charlesworth ${ }^{73}$, R. Sandhu ${ }^{73}$, M. Thirumaran ${ }^{73}$, V. Wagstaff ${ }^{73}$ \& J. Cebrian Suarez ${ }^{73}$

Basildon Hospital, Basildon, UK

A. Kaliappan ${ }^{74}$, M. Vertue ${ }^{74}$, A. Nicholson ${ }^{74}$, J. Riches ${ }^{74}$, A. Solesbury ${ }^{74}$, L. Kittridge ${ }^{74}$,

M. Forsey ${ }^{74}$ \& G. Maloney ${ }^{74}$ 


\section{Article}

University Hospital of Wales, Cardiff, UK

J. Cole ${ }^{75}$, M. Davies ${ }^{75}$, R. Davies ${ }^{75}$, H. Hill ${ }^{75}$, E. Thomas ${ }^{75}$, A. Williams ${ }^{75}$, D. Duffin ${ }^{75}$ \& B. Player ${ }^{75}$

Broomfield Hospital, Chelmsford, UK

J. Radhakrishnan ${ }^{76}$, S. Gibson ${ }^{76}$, A. Lyle ${ }^{76}$ \& F. McNeela ${ }^{76}$

Royal Brompton Hospital, London, UK

B. Patel ${ }^{77}$, M. Gummadi ${ }^{77}$, G. Sloane ${ }^{77}$, N. Dormand ${ }^{77}$, S. Salmi ${ }^{77}$, Z. Farzad ${ }^{77}$, D. Cristiano ${ }^{77}$,

K. Liyanage ${ }^{77}$, V. Thwaites ${ }^{77}$ \& M. Varghese ${ }^{77}$

Nottingham University Hospital, Nottingham, UK

M. Meredith ${ }^{78}$

Royal Hallamshire Hospital \& Northern General Hospital, Sheffield, UK

G. Mills ${ }^{79}$, J. Willson ${ }^{79}$, K. Harrington ${ }^{79}$, B. Lenagh ${ }^{79}$, K. Cawthron $^{79}$, S. Masuko ${ }^{79}$,

A. Raithatha ${ }^{79}$, K. Bauchmuller ${ }^{79}$, M. Wiles ${ }^{79}$, N. Ahmad ${ }^{79}$, J. Barker ${ }^{79}$, Y. Jackson ${ }^{79}$, F. Kibutu ${ }^{79}$ \&S. Bird ${ }^{79}$

Royal Hampshire County Hospital, Hampshire, UK

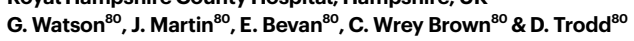

Queens Hospital Burton, Burton-On-Trent, UK

K. English ${ }^{81}$, G. Bell ${ }^{81}$, L. Wilcox ${ }^{81}$ \& A. Katary ${ }^{81}$

New Cross Hospital, Wolverhampton, UK

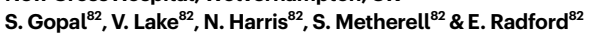

Heartlands Hospital, Birmingham, UK

J. Scriven ${ }^{83}$, F. Moore ${ }^{83}$, H. Bancroft ${ }^{83}$, J. Daglish ${ }^{83}$, M. Sangombe ${ }^{83}$, M. Carmody ${ }^{83}$,

J. Rhodes ${ }^{83}$ \& M. Bellamy ${ }^{83}$

Walsall Manor Hospital, Walsall, UK

A. Garg ${ }^{84}$, A. Kuravi ${ }^{84}$, E. Virgilio ${ }^{84}$, P. Ranga ${ }^{84}$, J. Butler ${ }^{84}$, L. Botfield ${ }^{84}$, C. Dexter ${ }^{84}$ \&

J. Fletcher ${ }^{84}$

Stoke Mandeville Hospital, Buckinghamshire, UK

P. Shanmugasundaram ${ }^{85}$, G. Hambrook ${ }^{85}$, I. Burn ${ }^{85}$, K. Manso $^{85}$, D. Thornton ${ }^{85}$, J. Tebbutt ${ }^{85}$ \&

R. Penn ${ }^{85}$

Sandwell General Hospital, Birmingham, UK

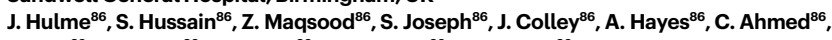

R. $\mathrm{Haq}^{86}$, S. Clamp ${ }^{86}$, R. Kumar ${ }^{86}$, M. Purewal ${ }^{86}$ \& B. Baines ${ }^{86}$

Royal Berkshire NHS Foundation Trust, Berkshire, UK

M. Frise ${ }^{87}$, N. Jacques ${ }^{87}$, H. Coles $^{87}$, J. Caterson ${ }^{87}$, S. Gurung Rai ${ }^{87}$, M. Brunton ${ }^{87}$, E. Tilney ${ }^{87}$

L. Keating ${ }^{87}$ \& A. Walden ${ }^{87}$

Charing Cross Hospital, St Mary's Hospital \& Hammersmith Hospital, London, UK

D. Antcliffe ${ }^{88}$, S. Brett ${ }^{88}$, A. Gordon ${ }^{88}$, M. Templeton ${ }^{88}$, R. Rojo ${ }^{88}$, D. Banach ${ }^{88}$, S. Sousa Arias ${ }^{88}$,

Z. Fernandez ${ }^{88} \&$ P. Coghlan ${ }^{88}$

Dumfries \& Galloway Royal Infirmary, Dumfries, UK

D. Williams ${ }^{89} \&$ C. Jardine ${ }^{89}$

Bristol Royal Infirmary, Bristol, UK

J. Bewley ${ }^{90}$, K. Sweet ${ }^{90}$, L. Grimmer ${ }^{90}$, R. Johnson ${ }^{90}$, Z. Garland ${ }^{90}$ \& B. Gumbrill ${ }^{90}$

Royal Sussex County Hospital, Brighton, UK

C. Phillips ${ }^{91}$, L. Ortiz-Ruiz de Gordoa ${ }^{91}$ \& E. Peasgood ${ }^{91}$

Whiston Hospital, Prescot, UK

A. Tridente ${ }^{92}$, K. Shuker ${ }^{92} \&$ S. Greer ${ }^{92}$

Royal Glamorgan Hospital, Cardiff, UK

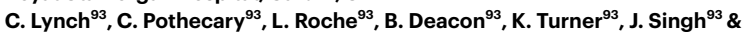

G. Sera Howe ${ }^{93}$

King's Mill Hospital, Nottingham, UK

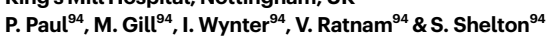

Fairfield General Hospital, Bury, UK

J. Naisbitt ${ }^{95}$ \& J. Melville ${ }^{95}$

Western General Hospital, Edinburgh, UK

R. Baruah ${ }^{96} \&$ S. Morrison ${ }^{96}$

Northwick Park Hospital, London, UK

A. McGregor ${ }^{97}$, V. Parris ${ }^{97}$, M. Mpelembue ${ }^{97}$, S. Srikaran ${ }^{97}$, C. Dennis ${ }^{97}$ \& A. Sukha ${ }^{97}$
Royal Preston Hospital, Preston, UK

A. Williams ${ }^{98} \&$ M. Verlander ${ }^{98}$

Royal Derby Hospital, Derby, UK

K. Holding ${ }^{99}$, K. Riches ${ }^{99}$, C. Downes ${ }^{99}$ \& C. Swan ${ }^{99}$

Sunderland Royal Hospital, Sunderland, UK

A. Rostron ${ }^{100}$, A. Roy ${ }^{100}$, L. Woods ${ }^{100}$, S. Cornell ${ }^{100}$ \& F. Wakinshaw ${ }^{100}$

Royal Surrey County Hospital, Guildford, UK

B. Creagh-Brown ${ }^{101}$, H. Blackman ${ }^{101}$, A. Salberg ${ }^{101}$, E. Smith ${ }^{101}$, S. Donlon ${ }^{101}$, S. Mtuwa ${ }^{101}$

N. Michalak-Glinska ${ }^{101}$, S. Stone ${ }^{101}$, C. Beazley ${ }^{101}$ \& V. Pristopan ${ }^{101}$

Derriford Hospital, Plymouth, UK

N. Nikitas ${ }^{102}$, L. Lankester ${ }^{102}$ \& C. Wells ${ }^{102}$

Croydon University Hospital, Croydon, UK

A.S. Raj ${ }^{103}$, K. Fletcher ${ }^{103}$, R. Khade ${ }^{103}$ \& G. Tsinaslanidis ${ }^{103}$

Victoria Hospital, Kirkcaldy, UK

M. MacMahon ${ }^{104}$, S. Fowler ${ }^{104}$, A. McGregor ${ }^{104}$ \& T. Coventry ${ }^{104}$

Milton Keynes University Hospital, Milton Keynes, UK

R. Stewart ${ }^{105}$, L. Wren ${ }^{105}$, E. Mwaura ${ }^{105}$, L. Mew ${ }^{105}$, A. Rose ${ }^{105}$, D. Scaletta ${ }^{105}$ \& F. Williams ${ }^{105}$

Barnsley Hospital, Barnsley, UK

K. Inweregbu ${ }^{106}$, A. Nicholson ${ }^{106}$, N. Lancaster ${ }^{106}$, M. Cunningham ${ }^{106}$, A. Daniels ${ }^{106}$,

L. Harrison ${ }^{106}$, S. Hope ${ }^{106}$, S. Jones ${ }^{106}$, A. Crew ${ }^{106}$, G. Wray ${ }^{106}$, J. Matthews ${ }^{106}$ \&

R. Crawley ${ }^{106}$

York Hospital, York, UK

J. Carter ${ }^{107}$, I. Birkinshaw ${ }^{107}$, J. Ingham ${ }^{107}$, Z. Scott ${ }^{107}$, H. Pearson ${ }^{107}$, K. Howard ${ }^{107}$, R. Joy ${ }^{107}$ \&

S. Roche ${ }^{107}$

University Hospital of North Tees, Stockton on Tees, UK

M. Clark ${ }^{108} \&$ S. Purvis ${ }^{108}$

University Hospital Wishaw, Wishaw, UK

A. Morrison ${ }^{109}$, D. Strachan ${ }^{109}$, M. Taylor ${ }^{109}$, S. Clements ${ }^{109}$ \& K. Black ${ }^{109}$

Whittington Hospital, London, UK

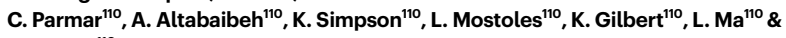

A. Alvaro"10

Southmead Hospital, Bristol, UK

M. Thomas ${ }^{111}$, B. Faulkner ${ }^{111}$, R. Worner ${ }^{111}$, K. Hayes ${ }^{111}$, E. Gendall ${ }^{111}$, H. Blakemore ${ }^{111}$,

B. Borislavova ${ }^{111}$ \& E. Goff ${ }^{111}$

The Royal Papworth Hospital, Cambridge, UK

A. Vuylsteke ${ }^{112}$, L. Mwaura ${ }^{12}$, J. Zamikula ${ }^{112}$, L. Garner ${ }^{112}$, A. Mitchell ${ }^{112}$, S. Mepham ${ }^{112}$,

L. Cagova ${ }^{112}$, A. Fofano ${ }^{112}$, H. Holcombe ${ }^{112}$ \& K. Praman ${ }^{112}$

Royal Gwent Hospital, Newport, UK

T. Szakmany ${ }^{113}$, A. E. Heron ${ }^{113}$, S. Cherian ${ }^{113}$, S. Cutler ${ }^{113}$ \& A. Roynon-Reed ${ }^{113}$

Norfolk \& Norwich University Hospital (NNUH), Norwich, UK

G. Randell ${ }^{114}$, K. Convery ${ }^{114}$, K. Stammers ${ }^{114}$, D. Fottrell-Gould ${ }^{114}$, L. Hudig $^{114}$ \&

J. Keshet-Price ${ }^{114}$

Great Ormond Street Hospital and UCL Great Ormond Street Institute of Child Health NIHR Biomedical Research Centre, London, UK

M. Peters ${ }^{115}$, L. O'Neill ${ }^{115}$, S. Ray ${ }^{115}$, H. Belfield ${ }^{115}$, T. McHugh ${ }^{115}$, G. Jones ${ }^{115}$, O. Akinkugbe ${ }^{115}$

A. Tomas ${ }^{115}$, E. Abaleke ${ }^{115}$, E. Beech ${ }^{115}$, H. Meghari ${ }^{115}$, S. Yussuf ${ }^{15}$ \& A. Bamford ${ }^{115}$

Airedale General Hospital, Keighley, UK

B. Hairsine ${ }^{116}$, E. Dooks ${ }^{116}$, F. Farquhar ${ }^{116}$, S. Packham ${ }^{116}$, H. Bates ${ }^{116}$, C. McParland ${ }^{116}$ \&

L. Armstrong ${ }^{116}$

Aberdeen Royal Infirmary, Aberdeen, UK

C. Kaye $^{117}$, A. Allan ${ }^{117}$, J. Medhora ${ }^{117}$, J. Liew ${ }^{117}$, A. Botello ${ }^{117}$ \& F. Anderson ${ }^{117}$

Southampton General Hospital, Southampton, UK

R. Cusack ${ }^{118}$, H. Golding ${ }^{118}$, K. Prager ${ }^{118}$, T. Williams ${ }^{118}$, S. Leggett ${ }^{118}$, K. Golder $^{118}$, M. Male ${ }^{118}$,

O. Jones ${ }^{118}$, K. Criste ${ }^{118}$ \& M. Marani ${ }^{118}$

Russell's Hall Hospital, Dudley, UK

V. Anumakonda ${ }^{119}$, V. Amin ${ }^{119}$, K. Karthik ${ }^{119}$, R. Kausar ${ }^{119}$, E. Anastasescu ${ }^{119}$, K. Reid ${ }^{119}$ \&

M. Smith ${ }^{119}$ 
Rotherham General Hospital, Rotherham, UK

A. Hormis ${ }^{120}$, R. Walker ${ }^{120} \&$ D. Collier ${ }^{120}$

North Manchester General Hospital, Manchester, UK

T. Duncan ${ }^{121}$, A. Uriel ${ }^{121}$, A. Ustianowski ${ }^{121}$, H. T-Michael ${ }^{121}$, M. Bruce $^{121}$, K. Connolly $^{121}$ \&

K. Smith ${ }^{121}$

Basingstoke \& North Hampshire Hospital, Basingstoke, UK

R. Partridge ${ }^{122}$, D. Griffin ${ }^{122}$, M. Mupudzi ${ }^{122} \&$ N. Muchenje ${ }^{122}$

Royal Free Hospital, London, UK

D. Martin ${ }^{123}$, H. Filipe ${ }^{123}$, C. Eastgate ${ }^{123}$ \& C. Jackson ${ }^{123}$

Hull Royal Infirmary, Hull, UK

A. Gratrix ${ }^{124}$, L. Foster ${ }^{124}$, V. Martinson ${ }^{124}$, E. Stones ${ }^{124}$, Caroline Abernathy ${ }^{124}$ \& P. Parkinson ${ }^{124}$

Harefield Hospital, London, UK

A. Reed ${ }^{125}$, C. Prendergast ${ }^{125}$, P. Rogers ${ }^{125}$, M. Woodruff ${ }^{125}$, R. Shokkar ${ }^{125}$, S. Kaul ${ }^{125}$,

A. Barron ${ }^{125} \&$ C. Collins ${ }^{125}$

Chesterfield Royal Hospital Foundation Trust, Chesterfield, UK

S. Beavis ${ }^{126}$, A. Whileman ${ }^{126}$, K. Dale ${ }^{126}$, J. Hawes ${ }^{126}$, K. Pritchard $^{126}$, R. Gascoyne ${ }^{126}$ \&

L. Stevenson ${ }^{126}$

Barnet Hospital, London, UK

R. Jha ${ }^{127}$, L. Lim ${ }^{127}$ \& V. Krishnamurthy ${ }^{127}$

Aintree University Hospital, Liverpool, UK

R. Parker ${ }^{128}$, I. Turner-Bone ${ }^{128}$, L. Wilding ${ }^{128}$ \& A. Reddy ${ }^{128}$

St James's University Hospital \& Leeds General Infirmary, Leeds, UK

S. Whiteley ${ }^{129}$, E. Wilby ${ }^{129}$, C. Howcroft ${ }^{129}$, A. Aspinwall ${ }^{129}$, S. Charlton ${ }^{129}$ \& B. Ogg ${ }^{129}$

Glan Clwyd Hospital, Bodelwyddan, UK

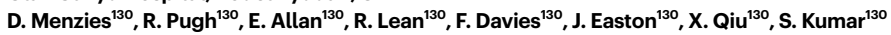
\& K. Darlington ${ }^{130}$

University Hospital Crosshouse, Kilmarnock, UK

G. Houston ${ }^{131}$, P. O'Brien ${ }^{131}$, T. Geary ${ }^{131}$, J. Allan ${ }^{131}$ \& A. Meikle ${ }^{131}$

Royal Bolton Hospital, Bolton, UK

G. Hughes ${ }^{132}$, M. Balasubramaniam ${ }^{132}$, S. Latham ${ }^{132}$, E. McKenna ${ }^{132}$ \& R. Flanagan ${ }^{132}$

Princess of Wales Hospital, Llantrisant, UK

S. Sathe ${ }^{133}$, E. Davies ${ }^{133} \&$ L. Roche ${ }^{133}$

Pilgrim Hospital, Lincoln, UK

M. Chablani ${ }^{134}$, A. Kirkby ${ }^{134}$, K. Netherton ${ }^{134}$ \& S. Archer ${ }^{134}$

Northumbria Healthcare NHS Foundation Trust, North Shields, UK

B. Yates ${ }^{135} \&$ C. Ashbrook-Raby ${ }^{135}$

Ninewells Hospital, Dundee, UK

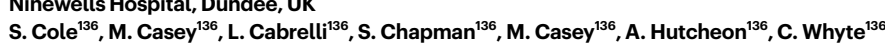
\& C. Almaden-Boyle ${ }^{136}$

Lister Hospital, Stevenage, UK

N. Pattison ${ }^{137} \&$ C. Cruz $^{137}$

Bedford Hospital, Bedford, UK

A. Vochin ${ }^{138}$, H. Kent ${ }^{138}$, A. Thomas ${ }^{138}$, S. Murdoch ${ }^{138}$, B. David ${ }^{138}$, M. Penacerrada ${ }^{138}$,

G. Lubimbi ${ }^{138}$, V. Bastion ${ }^{138}$, R. Wulandari ${ }^{138}$, R. Lorusso ${ }^{138}$, J. Valentine ${ }^{138}$ \& D. Clarke ${ }^{138}$

Royal United Hospital, Bath, UK

A. Serrano-Ruiz ${ }^{139}$, S. Hierons ${ }^{139}$, C. Eckbad ${ }^{139}$, L. $\operatorname{Ramos}^{139}$, C. Demetriou ${ }^{139}$, S. Mitchard $^{139}$ \&

K. White ${ }^{139}$

Royal Bournemouth Hospital, Bournemouth, UK

N. White ${ }^{140}$, S. Pitts ${ }^{140}$, D. Branney ${ }^{140}$ \& J. Frankham ${ }^{140}$

The Great Western Hospital, Swindon, UK

M. Watters ${ }^{141}$, H. Langton ${ }^{141} \&$ R. Prout ${ }^{141}$

Watford General Hospital, Watford, UK

V. Page $^{142} \&$ T. Varghes ${ }^{142}$

University Hospital North Durham, Darlington, UK

A. Cowton ${ }^{143}$, A. Kay ${ }^{143}$, K. Potts ${ }^{143}$, M. Birt ${ }^{143}$, M. Kent ${ }^{143}$ \& A. Wilkinson ${ }^{143}$
Tameside General Hospital, Ashton Under Lyne, UK

E. B. Jude ${ }^{144}$, V. Turner ${ }^{144}$, H. Savill ${ }^{144}$, J. McCormick ${ }^{144}$, M. Clark ${ }^{144}$, M. Coulding ${ }^{144}$,

S. Siddiquii ${ }^{144}$, O. Mercer $^{144}$, H. Rehman ${ }^{144}$ \& D. Potla ${ }^{144}$

Princess Royal Hospital, Telford \& Royal Shrewsbury Hospital, Shrewsbury, UK

N. Capps ${ }^{145}$, D. Donaldson ${ }^{145}$, J. Jones ${ }^{145}$, H. Button ${ }^{145}$, T. Martin ${ }^{145}$, K. Hard ${ }^{145}$, A. Agasou ${ }^{145}$

L. Tonks ${ }^{145}$, T. Arden ${ }^{145}$, P. Boyle ${ }^{145}$, M. Carnahan ${ }^{145}$, J. Strickley ${ }^{145}$, C. Adams ${ }^{145}$, D. Childs ${ }^{145}$

R. Rikunenko ${ }^{145}$, M. Leigh ${ }^{145}$, M. Breekes ${ }^{145}$, R. Wilcox ${ }^{145}$, A. Bowes ${ }^{145}$, H. Tiveran ${ }^{145}$,

F. Hurford ${ }^{145}$, J. Summers ${ }^{145}$, A. Carter ${ }^{145}$, Y. Hussain ${ }^{145}$, L. Ting $^{145}$, A. Javaid ${ }^{145}$,

N. Motherwell ${ }^{145}$, H. Moore ${ }^{145}$, H. Millward ${ }^{145}$, S. Jose ${ }^{145}$, N. Schunki ${ }^{145}$, A. Noakes $^{145}$ \&

C. Clulow ${ }^{145}$

Arrowe Park Hospital, Wirral, UK

G. Sadera ${ }^{146}$, R. Jacob ${ }^{146} \&$ C. Jones ${ }^{146}$

The Queen Elizabeth Hospital, King's Lynn, UK

M. Blunt ${ }^{147}$, Z. Coton ${ }^{147}$, H. Curgenven ${ }^{147}$, S. Mohamed Ally ${ }^{147}$, K. Beaumont ${ }^{147}$,

M. Elsaadany ${ }^{147}$, K. Fernandes ${ }^{147}$, I. Ali Mohamed $\mathrm{Ali}^{147}$, H. Rangarajan ${ }^{147}$, V. Sarathy ${ }^{147}$,

S. Selvanayagam ${ }^{147}$, D. Vedage ${ }^{147}$ \& M. White ${ }^{147}$

Royal Blackburn Teaching Hospital, Blackburn, UK

M. Smith ${ }^{148}$, N. Truman $^{148}$, S. Chukkambotla ${ }^{148}$, S. Keith ${ }^{148}$, J. Cockerill-Taylor ${ }^{148}$,

J. Ryan-Smith ${ }^{148}$, R. Bolton ${ }^{148}$, P. Springle ${ }^{148}$, J. Dykes ${ }^{148}$, J. Thomas $^{148}{ }^{\text {, M. Khan }}{ }^{148}$,

M. T. Hijazi' ${ }^{148}$, E. Massey ${ }^{148}$ \& G. Croston ${ }^{148}$

Poole Hospital, Poole, UK

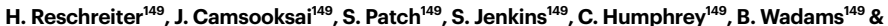

J. Camsooksai ${ }^{149}$

Medway Maritime Hospital, Gillingham, UK

M. Msiska ${ }^{150}$ \& O. Adanini ${ }^{150}$

Warwick Hospital, Warwick, UK

B. Attwood ${ }^{151}$ \& P. Parsons ${ }^{151}$

The Royal Marsden Hospital, London, UK

K. Tatham ${ }^{152}$, S. Jhanji ${ }^{152}$, E. Black ${ }^{152}$, A. Dela Rosa ${ }^{152}$, R. Howle ${ }^{152}$, B. Thomas ${ }^{152}$, T. Bemand ${ }^{152}$ \& R. Raobaikady ${ }^{152}$

The Princess Alexandra Hospital, Harlow, UK

R. Saha ${ }^{153}$, N. Staines ${ }^{153}$, A. Daniel ${ }^{153} \&$ J. Finn ${ }^{153}$

Musgrove Park Hospital, Taunton, UK

J. Hutter ${ }^{154}$, P. Doble ${ }^{154}$, C. Shovelton ${ }^{154}$ \& C. Pawley ${ }^{154}$

George Eliot Hospital NHS Trust, Nuneaton, UK

T. Kannan ${ }^{155}$ \& M. Hill ${ }^{155}$

East Surrey Hospital, Redhill, UK

E. Combes ${ }^{156}$, S. Monnery ${ }^{156} \&$ T. Joefield ${ }^{156}$

West Middlesex Hospital, Isleworth, UK

M. Popescu ${ }^{157}$, M. Thankachen ${ }^{157}$ \& M. Oblak ${ }^{157}$

Warrington General Hospital, Warrington, UK

J. Little ${ }^{158}$, S. Mclvor ${ }^{158}$, A. Brady ${ }^{158}$, H. Whittle ${ }^{158}$, H. Prady ${ }^{158}$ \& R. Chan ${ }^{158}$

Southport \& Formby District General Hospital, Ormskirk, UK

A. Ahmed ${ }^{159}$ \& A. Morris ${ }^{159}$

Royal Devon \& Exeter Hospital, Exeter, UK

C. Gibson ${ }^{160}$, E. Gordon ${ }^{160}$, S. Keenan ${ }^{160}$, H. Quinn ${ }^{160}$, S. Benyon ${ }^{160}$, S. Marriott ${ }^{160}$, L. Zitter ${ }^{160}$,

L. Park $^{160} \&$ K. Baines ${ }^{160}$

Macclesfield District General Hospital, Macclesfield, UK

M. Lyons ${ }^{161}$, M. Holland ${ }^{161}$, N. Keenan ${ }^{161}$ \& M. Young ${ }^{161}$

Borders General Hospital, Melrose, UK

S. Garrioch ${ }^{162}$, J. Dawson ${ }^{162} \&$ M. Tolson ${ }^{162}$

Birmingham Children's Hospital, Birmingham, UK

B. Scholefield ${ }^{163} \&$ R. Bi ${ }^{163}$

William Harvey Hospital, Ashford, UK

N. Richardson ${ }^{164}$, N. Schumacher ${ }^{164}$, T. Cosier $^{164}$ \& G. Millen ${ }^{164}$

Royal Lancaster Infirmary, Lancaster, UK

A. Higham ${ }^{165} \&$ K. Simpson ${ }^{165}$ 


\section{Article}

Queen Elizabeth the Queen Mother Hospital, Margate, UK

S. Turki ${ }^{166}$, L. Allen ${ }^{166}$, N. Crisp ${ }^{166}$, T. Hazleton ${ }^{166}$, A. Knight $^{166}$, J. Deery ${ }^{166}$, C. Price ${ }^{166}$

S. Turney ${ }^{166}$, S. Tilbey ${ }^{166} \&$ E. Beranova ${ }^{166}$

Liverpool Heart \& Chest Hospital, Liverpool, UK

D. Wright ${ }^{167}$, L. George ${ }^{167}$ \& S. Twiss ${ }^{167}$

Darlington Memorial Hospital, Darlington, UK

A. Cowton ${ }^{168}$, S. Wadd ${ }^{168} \&$ K. Postlethwaite ${ }^{168}$

Southend University Hospital, Westcliff-on-Sea, UK

P. Gondo ${ }^{169}$, B. Masunda ${ }^{169}$, A. Kayani ${ }^{169}$ \& B. Hadebe ${ }^{169}$

Raigmore Hospital, Inverness, UK

J. Whiteside ${ }^{170}$, R. Campbell ${ }^{170} \&$ N. Clarke ${ }^{170}$

Salisbury District Hospital, Salisbury, UK

P. Donnison ${ }^{171}$, F. Trim $^{171}$ \& I. Leadbitter ${ }^{171}$

Peterborough City Hospital, Peterborough, UK

D. Butcher ${ }^{172} \&$ S. O'Sullivan ${ }^{172}$

Ipswich Hospital, Ipswich, UK

B. Purewal ${ }^{173}$, S. Bell ${ }^{173} \&$ V. Rivers ${ }^{173}$

Hereford County Hospital, Hereford, UK

R. O'Leary ${ }^{174}$, J. Birch ${ }^{174}$, E. Collins ${ }^{174}$, S. Anderson ${ }^{174}$, K. Hammerton ${ }^{174}$ \& E. Andrews ${ }^{174}$

Furness General Hospital, Barrow-in-Furness, UK

A. Higham ${ }^{175} \&$ K. Burns ${ }^{175}$

Forth Valley Royal Hospital, Falkirk, UK

I. Edmond ${ }^{176}$, D. Salutous ${ }^{176}$, A. Todd ${ }^{176}$, J. Donnachie ${ }^{176}$, P. Turner ${ }^{176}$, L. Prentice ${ }^{176}$, L. Symon ${ }^{176}$,

N. Runciman ${ }^{176}$ \& F. Auld ${ }^{176}$

Torbay Hospital, Torquay, UK

M. Halkes ${ }^{177}$, P. Mercer ${ }^{177}$ \& L. Thornton ${ }^{177}$

St Mary's Hospital, Newport, UK

G. Debreceni $^{178}$, J. Wilkins ${ }^{178}$, A. Brown ${ }^{178}$ \& V. Crickmore ${ }^{178}$

Royal Manchester Children's Hospital, Manchester, UK

G. Subramanian ${ }^{179}$, R. Marshall ${ }^{179}$, C. Jennings ${ }^{179}$, M. Latif ${ }^{179}$ \& L. Bunni ${ }^{179}$

Royal Cornwall Hospital, Truro, UK

M. Spivey ${ }^{180}$, S. Bean ${ }^{180} \&$ K. Burt ${ }^{180}$

Queen Elizabeth Hospital Gateshead, Gateshead, UK

V. Linnett ${ }^{181}$, J. Ritzema ${ }^{181}$, A. Sanderson ${ }^{181}$, W. McCormick ${ }^{181}$ \& M. Bokhari ${ }^{181}$

Kent \& Canterbury Hospital, Canterbury, UK

R. Kapoor ${ }^{182} \&$ D. Loader $^{182}$

James Paget University Hospital NHS Trust, Great Yarmouth, UK

A. Ayers ${ }^{183}$, W. Harrison ${ }^{183}$ \& J. North ${ }^{183}$

Darent Valley Hospital, Dartford, UK

Z. Belagodu ${ }^{184}$, R. Paramsothy ${ }^{184}$, O. Olufuwa ${ }^{184}$, A. Gherman ${ }^{184}$, B. Fuller ${ }^{184}$ \& C. Stuart ${ }^{184}$

Alexandra Hospital, Redditch \& Worcester Royal Hospital, Worcester, UK

O. Kelsall ${ }^{185}$, C. Davis ${ }^{185}$, L. Wild ${ }^{185}$, H. Wood ${ }^{185}$, J. Thrush ${ }^{185}$, A. Durie ${ }^{185}$, K. Austin ${ }^{185}$,

K. Archer ${ }^{185}$, P. Anderson ${ }^{185}$ \& C. Vigurs ${ }^{185}$

Ysbyty Gwynedd, Bangor, UK

C. Thorpe ${ }^{186}$, A. Thomas ${ }^{186}$, E. Knights ${ }^{186}$, N. Boyle ${ }^{186}$ \& A. Price ${ }^{186}$

Yeovil Hospital, Yeovil, UK

A. Kubisz-Pudelko ${ }^{187}$, D. Wood ${ }^{187}$, A. Lewis ${ }^{187}$, S. Board ${ }^{187}$, L. Pippard ${ }^{187}$, J. Perry ${ }^{187}$ \& K. Beesley ${ }^{187}$

University Hospital Hairmyres, East Kilbride, UK

A. Rattray ${ }^{188}$, M. Taylor $^{188}$, E. Lee ${ }^{188}$, L. Lennon ${ }^{188}$, K. Douglas ${ }^{188}$, D. Bell ${ }^{188}$, R. Boyle ${ }^{188}$ \&

L. Glass ${ }^{188}$

Scunthorpe General Hospital, Scunthorpe, UK

M. Nauman Akhtar ${ }^{189}$, K. Dent ${ }^{189}$, D. Potoczna ${ }^{189}$, S. Pearson ${ }^{189}$, E. Horsley ${ }^{189}$ \& S. Spencer ${ }^{189}$

Princess Royal Hospital Brighton, West Sussex, UK

C. Phillips ${ }^{190}$, D. Mullan ${ }^{190}$, D. Skinner ${ }^{190}$, J. Gaylard ${ }^{190}$ \& L. Ortiz-Ruiz de Gordoa ${ }^{190}$
Lincoln County Hospital, Lincoln, UK

R. Barber ${ }^{191}$, C. Hewitt ${ }^{191}$, A. Hilldrith ${ }^{191}$, S. Shepardson ${ }^{191}$, M. Wills ${ }^{191}$ \& K. Jackson-Lawrence ${ }^{191}$

Homerton University Hospital, London, UK

A. Gupta ${ }^{192}$, A. Easthope ${ }^{192}$, E. Timlick ${ }^{122}$ \& C. Gorman ${ }^{192}$

Glangwili General Hospital, Camarthen, UK

I. Otahal ${ }^{193}$, A. Gales ${ }^{193}$, S. Coetzee ${ }^{193}$, C. Sell ${ }^{193}$, M. Raj ${ }^{193}$ \& M. Peiu ${ }^{193}$

Ealing Hospital, Southall, UK

V. Parris ${ }^{194}$, S. Quaid ${ }^{194}$ \& E. Watson ${ }^{194}$

Scarborough General Hospital, Scarborough, UK

K. Elliott ${ }^{195}$, J. Mallinson ${ }^{195}$, B. Chandler ${ }^{195}$ \& A. Turnbull ${ }^{195}$

Royal Albert Edward Infirmary, Wigan, UK

A. Quinn ${ }^{196}$, C. Finch ${ }^{196}$, C. Holl ${ }^{196}$, J. Cooper ${ }^{196}$ \& A. Evans ${ }^{196}$

Queen Elizabeth Hospital, Woolwich, London, UK

W. Khaliq ${ }^{197}$, A. Collins ${ }^{197}$ \& E. Treus Gude ${ }^{197}$

North Devon District Hospital, Barnstaple, UK

N. Love ${ }^{198}$, L. van Koutrik ${ }^{198}$, J. Hunt ${ }^{198}$, D. Kaye ${ }^{198}$, E. Fisher ${ }^{198}$, A. Brayne ${ }^{198}$, V. Tuckey ${ }^{198}$,

P. Jackson ${ }^{198}$ \& J. Parkin ${ }^{198}$

National Hospital for Neurology \& Neurosurgery, London, UK

D. Brealey ${ }^{199}$, E. Raith ${ }^{199}$, A. Tariq ${ }^{199}$, H. Houlden ${ }^{199}$, A. Tucci ${ }^{199}$, J. Hardy ${ }^{199}$ \& E. Moncur ${ }^{199}$

Eastbourne District General Hospital, East Sussex, UK \& Conquest Hospital, East Sussex, UK J. Highgate ${ }^{200} \&$ A. Cowley ${ }^{200}$

Diana Princess of Wales Hospital, Grimsby, UK

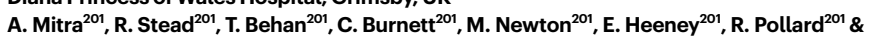
J. Hatton ${ }^{201}$

The Christie NHS Foundation Trust, Manchester, UK

A. Patel ${ }^{202}$, V. Kasipandian ${ }^{202}$, S. Allibone ${ }^{202}$ \& R. M. Genetu ${ }^{202}$

Prince Philip Hospital, Lianelli, UK

I. Otahal ${ }^{203}$, L. O'Brien ${ }^{203}$, Z. Omar ${ }^{203}$, E. Perkins ${ }^{203} \&$ K. Davies ${ }^{203}$

Prince Charles Hospital, Merthyr Tydfil, UK

D. Tetla ${ }^{204}$, C. Pothecary ${ }^{204} \&$ B. Deacon ${ }^{204}$

Golden Jubilee National Hospital, Clydebank, UK

B. Shelley ${ }^{205} \&$ V. Irvine ${ }^{205}$

Dorset County Hospital, Dorchester, UK

S. Williams ${ }^{206}$, P. Williams ${ }^{206}$, J. Birch ${ }^{206}$, J. Goodsell ${ }^{206}$, R. Tutton ${ }^{206}$, L. Bough ${ }^{206}$ \&

B. Winter-Goodwin ${ }^{206}$

Calderdale Royal Hospital, Halifax, UK

R. Kitson ${ }^{207}$, J. Pinnell ${ }^{207}$, A. Wilson ${ }^{207}$, T. Nortcliffe ${ }^{207}$, T. Wood ${ }^{207}$, M. Home ${ }^{207}$, K. Holdroyd ${ }^{207}$,

M. Robinson ${ }^{207}$, K. Hanson ${ }^{207}$, R. Shaw ${ }^{207}$, J. Greig ${ }^{207}$, M. Brady ${ }^{207}$, A. Haigh ${ }^{207}$, L. Matupe ${ }^{207}$,

M. Usher ${ }^{207}$, S. Mellor ${ }^{207}$, S. Dale ${ }^{207}$, L. Gledhill ${ }^{207}$, L. Shaw ${ }^{207}$, G. Turner ${ }^{207}$, D. Kelly ${ }^{207}$,

B. Anwar ${ }^{207}$, H. Riley ${ }^{207}$, H. Sturgeon ${ }^{207}$, A. Ali ${ }^{207}$, L. Thomis ${ }^{207}$, D. Melia ${ }^{207}$ \& A. Dance ${ }^{207}$

West Suffolk Hospital, Suffolk, UK

S. Humphreys ${ }^{208}$, I. Frost ${ }^{208}$, V. Gopal ${ }^{208}$, J. Godden ${ }^{208}$, A. Holden ${ }^{208} \&$ S. Swann ${ }^{208}$

West Cumberland Hospital, Whitehaven, UK

T. Smith ${ }^{209}$, M. Clapham ${ }^{209}$, U. Poultney ${ }^{209}$, R. Harper $^{209}$ \& P. Rice ${ }^{209}$

University Hospital Lewisham, London, UK

W. Khaliq ${ }^{210}$, R. Reece-Anthony ${ }^{210}$ \& B. Gurung ${ }^{210}$

St John's Hospital Livingston, Livingston, UK

S. Moultrie ${ }^{211} \&$ M. Odam ${ }^{21}$

Sheffield Children's Hospital, Sheffield, UK

A. Mayer ${ }^{212}$, A. Bellini ${ }^{212}$, A. Pickard ${ }^{212}$, J. Bryant ${ }^{212}$, N. Roe ${ }^{212}$ \& J. Sowter ${ }^{212}$

Hinchingbrooke Hospital, Huntingdon, UK

D. Butcher ${ }^{213}$ K. Lang ${ }^{213} \&$ J. Taylor ${ }^{213}$

Glenfield Hospital, Leicester, UK

P. Barry ${ }^{214}$ 
Bronglais General Hospital, Aberystwyth, UK

M. Hobrok ${ }^{215}$, H. Tench ${ }^{215}$, R. Wolf-Roberts ${ }^{215}$, H. McGuinness ${ }^{215}$ \& R. Loosley ${ }^{215}$

Alder Hey Children's Hospital, Liverpool, UK

D. Hawcutt ${ }^{216}$, L. Rad $^{216}$, L. O'Malley ${ }^{216}$, P. Saunderson ${ }^{216}$, G. Seddon ${ }^{216}$, T. Anderson ${ }^{216}$ \& N. Rogers ${ }^{216}$

University Hospital Monklands, Airdrie, UK

J. Ruddy ${ }^{217}$, M. Harkins ${ }^{217}$, M. Taylor ${ }^{217}$, C. Beith ${ }^{217}$, A. McAlpine ${ }^{217}$, L. Ferguson ${ }^{217}$, P. Grant ${ }^{217}$ S. MacFadyen ${ }^{217}$, M. McLaughlin ${ }^{217}$, T. Baird ${ }^{217}$, S. Rundell ${ }^{217}$, L. Glass ${ }^{217}$, B. Welsh ${ }^{217}$, R. Hamill ${ }^{217}$ \& F. Fisher ${ }^{217}$

Cumberland Infirmary, Carlisle, UK

T. Smith ${ }^{218}$, J. Gregory ${ }^{218} \&$ A. Brown ${ }^{218}$

Betsi Cadwaladr University Health Board, Wrexham, Wales, UK A. Campbell ${ }^{219} \&$ S. Smuts ${ }^{219}$

${ }^{40}$ Barts Health NHS Trust, London, UK. ${ }^{41}$ Guy's and St Thomas' Hospital, London, UK. ${ }^{42}$ James Cook University Hospital, Middlesburgh, UK. ${ }^{43}$ The Royal Liverpool University Hospital, Liverpool, UK. ${ }^{44}$ King's College Hospital, London, UK. ${ }^{45}$ Royal Infirmary of Edinburgh Edinburgh, UK. ${ }^{46} \mathrm{John}$ Radcliffe Hospital, Oxford, UK. ${ }^{47}$ Addenbrooke's Hospital, Cambridge, UK. ${ }^{48}$ Morriston Hospital, Swansea, UK. ${ }^{49}$ Ashford and St Peter's Hospitals, Chertsey, UK. ${ }^{50}$ Royal Stoke University Hospital, Stoke-on-Trent, UK. ${ }^{51}$ Queen Elizabeth Hospital, Birmingham, UK. ${ }^{52}$ Glasgow Royal Infirmary, Glasgow, UK. ${ }^{53}$ Kingston Hospital, Kingston upon Thames, UK. ${ }^{54}$ The Tunbridge Wells Hospital and Maidstone Hospital, Maidstone, UK. ${ }^{55}$ North Middlesex University Hospital NHS Trust, London, UK. ${ }^{56}$ Bradford Royal Infirmary, Bradford, UK. ${ }^{57}$ Blackpool Victoria Hospital, Blackpool, UK. ${ }^{58}$ Countess of Chester Hospital, Chester, UK. ${ }^{59}$ Wythenshawe Hospital, Manchester, UK. ${ }^{60}$ St George's Hospital, London, UK. ${ }^{61}$ Good Hope Hospital, Birmingham, UK. ${ }^{62}$ Stepping Hill Hospital, Stockport, UK. ${ }^{63}$ Manchester Royal Infirmary, Manchester, UK. ${ }^{64}$ Royal Alexandra Hospital, Paisley, UK. ${ }^{65}$ Queen Elizabeth University Hospital, Glasgow, UK. ${ }^{66}$ Queen Alexandra Hospital, Portsmouth, UK. ${ }^{67}$ BHRUT (Barking Havering) - Queens Hospital and King George Hospital, Ilford, UK. ${ }^{68}$ University College Hospital, London, UK. ${ }^{69}$ Royal Victoria Infirmary, Newcastle Upon Tyne, UK. ${ }^{70}$ Western Sussex Hospitals, Chichester, UK. ${ }^{7}$ Salford Royal Hospital, Manchester, UK. ${ }^{72}$ The Royal Oldham Hospital, Manchester, UK. ${ }^{73}$ Pinderfields General Hospital, Wakefield, UK. ${ }^{74}$ Basildon Hospital, Basildon, UK. ${ }^{75}$ University Hospital of Wales, Cardiff, UK. ${ }^{76}$ Broomfield Hospital, Chelmsford, UK. ${ }^{77}$ Royal Brompton Hospital, London, UK. ${ }^{78}$ Nottingham University Hospital, Nottingham, UK. ${ }^{79}$ Royal Hallamshire Hospital and Northern General Hospital, Sheffield, UK. ${ }^{80}$ Royal Hampshire County Hospital, Winchester, UK. ${ }^{81}$ Queens Hospital Burton, Burton-On-Trent, UK. ${ }^{82} \mathrm{New}$ Cross Hospital, Wolverhampton, UK. ${ }^{83} \mathrm{Heartlands}$ Hospital, Birmingham, UK. ${ }^{84}$ Walsall Manor Hospital, Walsall, UK. ${ }^{85}$ Stoke Mandeville Hospital, Aylesbury, UK. ${ }^{86}$ Sandwell General Hospital, Birmingham, UK. ${ }^{87}$ Royal Berkshire NHS Foundation Trust, Reading, UK. ${ }^{88}$ Charing Cross Hospital, St Mary's Hospital and Hammersmith Hospital, London, UK. ${ }^{89}$ Dumfries and Galloway Royal Infirmary, Dumfries, UK. ${ }^{90}$ Bristol Royal Infirmary, Bristol, UK. ${ }^{91}$ Royal Sussex County Hospital, Brighton, UK. ${ }^{92}$ Whiston Hospital, Prescot, UK. ${ }^{93}$ Royal Glamorgan Hospital, Cardiff, UK. ${ }^{94}$ King's Mill Hospital, Nottingham, UK. ${ }^{95}$ Fairfield General Hospital, Bury, UK. ${ }^{96}$ Western General Hospital, Edinburgh, UK. ${ }^{97}$ Northwick Park Hospital, London, UK. ${ }^{98}$ Royal Preston Hospital, Preston, UK. ${ }^{99}$ Royal Derby Hospital, Derby, UK. ${ }^{100}$ Sunderland Royal Hospital, Sunderland, UK. ${ }^{101}$ Royal Surrey County Hospital, Guildford, UK. ${ }^{102}$ Derriford Hospital, Plymouth, UK. ${ }^{103}$ Croydon University Hospital, Croydon, UK. ${ }^{104}$ Victoria Hospital, Kirkcaldy, UK. ${ }^{105}$ Milton Keynes University Hospital, Milton Keynes, UK. ${ }^{106}$ Barnsley Hospital, Barnsley, UK. ${ }^{107}$ York Hospital, York, UK. ${ }^{108}$ University Hospital of North Tees, Stockton on Tees, UK. ${ }^{109}$ University Hospital Wishaw, Wishaw, UK. ${ }^{110}$ Whittington Hospital, London, UK. ${ }^{11}$ Southmead Hospital, Bristol, UK. ${ }^{112}$ The Royal Papworth Hospital, Cambridge, UK. ${ }^{113}$ Royal Gwent Hospital, Newport, UK. ${ }^{114}$ Norfolk and Norwich University Hospital (NNUH), Norwich, UK. ${ }^{115}$ Great Ormond St Hospital and UCL Great Ormond St Institute of Child Health NIHR Biomedical Research Centre London, UK. ${ }^{116}$ Airedale General Hospital, Keighley, UK. ${ }^{117}$ Aberdeen Royal Infirmary, Aberdeen, UK. ${ }^{118}$ Southampton General Hospital, Southampton, UK. ${ }^{119}$ Russell's Hall Hospital, Dudley, UK. ${ }^{120}$ Rotherham General Hospital, Rotherham, UK. ${ }^{121}$ North Manchester General Hospital, Manchester, UK. ${ }^{122}$ Basingstoke and North Hampshire Hospital, Basingstoke, UK. ${ }^{123}$ Royal Free Hospital, London, UK. ${ }^{124}$ Hull Royal Infirmary, Hull, UK. ${ }^{125}$ Harefield Hospital, London, UK. ${ }^{126}$ Chesterfield Royal Hospital Foundation Trust, Chesterfield, UK. ${ }^{127}$ Barnet Hospital, London UK. ${ }^{128}$ Aintree University Hospital, Liverpool, UK. ${ }^{129}$ St James's University Hospital and Leeds General Infirmary, Leeds, UK. ${ }^{130} \mathrm{Glan}$ Clwyd Hospital, Bodelwyddan, UK. ${ }^{131}$ University Hospital Crosshouse, Kilmarnock, UK. ${ }^{132}$ Royal Bolton Hospital, Bolton, UK. ${ }^{133}$ Princess of Wales Hospital, Llantrisant, UK. ${ }^{134}$ Pilgrim Hospital, Lincoln, UK. ${ }^{135}$ Northumbria Healthcare NHS Foundation Trust, North Shields, UK. ${ }^{136}$ Ninewells Hospital, Dundee, UK. ${ }^{137}$ Lister Hospital Stevenage, UK. ${ }^{138}$ Bedford Hospital, Bedford, UK. ${ }^{139}$ Royal United Hospital, Bath, UK. ${ }^{140}$ Royal Bournemouth Hospital, Bournemouth, UK. ${ }^{141}$ The Great Western Hospital, Swindon, UK. ${ }^{142}$ Watford General Hospital, Watford, UK. ${ }^{143}$ University Hospital North Durham, Darlington, UK. ${ }^{144}$ Tameside General Hospital, Ashton Under Lyne, UK. ${ }^{145}$ Princess Royal Hospital Shrewsbury and Royal Shrewsbury Hospital, Shrewsbury, UK. ${ }^{146}$ Arrowe Park Hospital, Wirral, UK. ${ }^{147}$ The Queen Elizabeth Hospital, King's Lynn, UK. ${ }^{148}$ Royal Blackburn Teaching Hospital, Blackburn, UK. ${ }^{149}$ Poole Hospital, Poole, UK. ${ }^{150}$ Medway Maritime Hospital, Gillingham, UK. ${ }^{151}$ Warwick Hospital, Warwick, UK. ${ }^{152}$ The Royal Marsden Hospital, London, UK. ${ }^{153}$ The Princess Alexandra Hospital, Harlow, UK. ${ }^{154}$ Musgrove Park Hospital, Taunton, UK. ${ }^{155}$ George Eliot Hospital NHS Trust, Nuneaton, UK. ${ }^{156}$ East Surrey Hospital, Redhill, UK. ${ }^{157}$ West Middlesex Hospital, Isleworth, UK. ${ }^{1{ }^{15}}$ Warrington General Hospital, Warrington, UK. ${ }^{159}$ Southport and Formby District General Hospital, Ormskirk, UK. ${ }^{160}$ Royal Devon and Exeter Hospital, Exeter, UK. ${ }^{161}$ Macclesfield District General Hospital, Macclesfield, UK. ${ }^{162}$ Borders General Hospital Melrose, UK. ${ }^{163}$ Birmingham Children's Hospital, Birmingham, UK. ${ }^{164}$ William Harvey Hospital, Ashford, UK. ${ }^{165}$ Royal Lancaster Infirmary, Lancaster, UK. ${ }^{166}$ Queen Elizabeth the Queen Mother Hospital, Margate, UK. ${ }^{167}$ Liverpool Heart and Chest Hospital, Liverpool, UK. ${ }^{168}$ Darlington
Memorial Hospital, Darlington, UK. ${ }^{169}$ Southend University Hospital, Westcliff-on-Sea, UK. ${ }^{170}$ Raigmore Hospital, Inverness, UK. ${ }^{171}$ Salisbury District Hospital, Salisbury, UK. ${ }^{172}$ Peterborough City Hospital, Peterborough, UK. ${ }^{173}$ Ipswich Hospital, Ipswich, UK. ${ }^{174}$ Hereford County Hospital, Worcester, UK. ${ }^{175}$ Furness General Hospital, Barrow-in-Furness, UK. ${ }^{176}$ Forth Valley Royal Hospital, Falkirk, UK. ${ }^{177}$ Torbay Hospital, Torquay, UK. ${ }^{178} \mathrm{St}$ Mary's Hospital, Newport, UK. ${ }^{179}$ Royal Manchester Children's Hospital, Manchester, UK. ${ }^{180}$ Royal Cornwall Hospital, Truro, UK. ${ }^{181}$ Queen Elizabeth Hospital Gateshead, Gateshead, UK. ${ }^{182}$ Kent \& Canterbury Hospital, Canterbury, UK. ${ }^{183}$ James Paget University Hospital NHS Trust, Great Yarmouth, UK. ${ }^{184}$ Darent Valley Hospital, Dartford, UK. ${ }^{185}$ The Alexandra Hospital, Redditch and Worcester Royal Hospital, Worcester, UK. ${ }^{186}$ Ysbyty Gwynedd, Bangor, UK. ${ }^{187}$ Yeovil Hospital, Yeovil, UK. ${ }^{188}$ University Hospital Hairmyres, East Kilbride, UK. ${ }^{189}$ Scunthorpe General Hospital, Scunthorpe, UK. ${ }^{190}$ Princess Royal Hospital Brighton, Haywards Heath, UK. ${ }^{191}$ Lincoln County Hospital, Lincoln, UK. ${ }^{192}$ Homerton University Hospital, London, UK. ${ }^{193} \mathrm{Glang}$ wili General Hospital, Camarthen, UK. ${ }^{194}$ Ealing Hospital, London, UK. ${ }^{195}$ Scarborough General Hospital, Scarborough, UK. ${ }^{196}$ Royal Albert Edward Infirmary, Wigan, UK. ${ }^{197}$ Queen Elizabeth Hospital, London, UK. ${ }^{198}$ North Devon District Hospital, Barnstaple, UK. ${ }^{199}$ National Hospital for Neurology and Neurosurgery, London, UK. ${ }^{200}$ Eastbourne District General Hospital, East Sussex, UK and Conquest Hospital, Eastbourne, UK. ${ }^{201}$ Diana Princess of Wales Hospital, Grimsby, UK. ${ }^{202}$ The Christie NHS Foundation Trust, Manchester, UK. ${ }^{203}$ Prince Philip Hospital, Lianelli, UK. ${ }^{204}$ Prince Charles Hospital, Merthyr Tydfil, UK. ${ }^{205} \mathrm{Golden}$ Jubilee National Hospital, Clydebank, UK. ${ }^{206}$ Dorset County Hospital, Dorchester, UK. ${ }^{207}$ Calderdale Royal Hospital, Halifax, UK. ${ }^{208}$ West Suffolk Hospital, Bury St Edmunds, UK. ${ }^{209}$ West Cumberland Hospital, Whitehaven, UK. ${ }^{210}$ University Hospital Lewisham, London, UK. ${ }^{211}$ St John's Hospital Livingston, Livingston, UK. ${ }^{212}$ Sheffield Children's Hospital, Sheffield, UK. ${ }^{213}$ Hinchingbrooke Hospital, Huntingdon, UK. ${ }^{214}$ Glenfield Hospital, Leicester, UK. ${ }^{215}$ Bronglais General Hospital, Aberystwyth, UK. ${ }^{216}$ Alder Hey Children's Hospital, Liverpool, UK. ${ }^{217}$ University Hospital Monklands, Airdrie, UK. ${ }^{218} \mathrm{Cumberland}$ Infirmary, Carlisle, UK. ${ }^{219} \mathrm{Betsi}$ Cadwaladr University Health Board, Wrexham, Wales, UK. A full list of members and their affiliations appears in the Supplementary Information.

\section{ISARIC4C Consortium}

\section{ISARIC co-investigators}

J. Kenneth Baillie ${ }^{1,2,11}$, Malcolm G. Semple ${ }^{18,39}$, Peter J. M. Openshaw ${ }^{37,38}$, Gail Carson ${ }^{220}$ Beatrice Alex ${ }^{221}$, Benjamin Bach ${ }^{221}$, Wendy S. Barclay ${ }^{222}$, Debby Bogaert ${ }^{4}$, Meera Chand ${ }^{223}$, Graham S. Cooke ${ }^{224}$, Annemarie B. Docherty ${ }^{11,225}$, Jake Dunning ${ }^{37,226}$, Ana da Silva Filipe ${ }^{227}$ ', Tom Fletcher ${ }^{228}$, Christoper A. Green ${ }^{229}$, Ewen M. Harrison ${ }^{225}$, Julian A. Hiscox ${ }^{230}$, Antonia Ying Wai Ho ${ }^{227,231}$, Peter W. Horby ${ }^{21}$, Samreen ljaz $^{232}$, Saye Khoo ${ }^{233}$, Paul Klenerman ${ }^{234,235}$, Andrew Law', Wei Shen Lim ${ }^{78}$, Alexander J. Mentzer ${ }^{234,236}$, Laura Merson $^{220}$, Alison M. Meynert ${ }^{2}$, Mahdad Noursadeghi ${ }^{237}$, Shona C. Moore ${ }^{238}$, Massimo Palmarini ${ }^{227}$, William A. Paxton ${ }^{238,239}$, Georgios Pollakis ${ }^{238,239}$, Nicholas Price ${ }^{240,241}$, Andrew Rambaut ${ }^{242}$, David L. Robertson ${ }^{227}$, Clark D. Russell ${ }^{14}$, Vanessa Sancho-Shimizu ${ }^{243}$, Janet T. Scott ${ }^{27,244}$, Thushan de Silva ${ }^{245}$, Louise Sigfrid ${ }^{220}$, Tom Solomon ${ }^{18,246}$, Shiranee Sriskandan 224,247 , David Stuart ${ }^{248}$, Charlotte Summers ${ }^{15}$, Richard S. Tedder ${ }^{249,250,251}$ Emma C. Thomson $^{227}$, A. A. Roger Thompson ${ }^{252}$, Ryan S. Thwaites ${ }^{37}$, Lance C. W. Turtle ${ }^{18,253}$ \& Maria Zambon ${ }^{226}$

\section{Project management team}

Hayley Hardwick ${ }^{18}$, Chloe Donohue ${ }^{254}$, Ruth Lyons' ${ }^{1}$, Fiona Griffiths' ${ }^{1}$ \& Wilna Oosthuyzen ${ }^{1}$

Data analysis team

Lisa Norman ${ }^{225}$, Riinu Pius ${ }^{225}$, Thomas M. Drake ${ }^{225}$, Cameron J. Fairfield ${ }^{225}$ Stephen R. Knight ${ }^{225}$, Kenneth A. Mclean ${ }^{225}$, Derek Murphy ${ }^{225}$ \& Catherine A. Shaw ${ }^{225}$

\section{Data architecture team}

Jo Dalton ${ }^{254}$, Michelle Girvan ${ }^{254}$, Egle Saviciute ${ }^{254}$, Stephanie Roberts ${ }^{254}$, Janet Harrison ${ }^{254}$, Laura Marsh $^{254}$, Marie Connor ${ }^{254}$, Sophie Halpin ${ }^{254}$, Clare Jackson ${ }^{254}$, Carrol Gamble ${ }^{254}$, Gary Leeming ${ }^{255}$, Andrew Law', Murray Wham ${ }^{2}$, Sara Clohisey ${ }^{1,329}$ \& Ross Hendry ${ }^{1}$

\section{Data analysis and management team}

William Greenhalf ${ }^{256}$, Victoria Shaw ${ }^{257}$, Sara McDonald ${ }^{227}$ \& Seán Keating ${ }^{11}$

${ }^{220}$ ISARIC Global Support Centre, Centre for Tropical Medicine and Global Health, Nuffield Department of Medicine, University of Oxford, Oxford, UK. ${ }^{221}$ School of Informatics, University of Edinburgh, Edinburgh, UK. ${ }^{222}$ Section of Molecular Virology, Imperial College London, London, UK. ${ }^{223}$ Antimicrobial Resistance and Hospital Acquired Infection Department, Public Health England, London, UK. ${ }^{224}$ Department of Infectious Disease, Imperial College London, London, UK. ${ }^{225}$ Centre for Medical Informatics, The Usher Institute, University of Edinburgh, Edinburgh, UK. ${ }^{226}$ National Infection Service, Public Health England, London, UK. ${ }^{227}$ MRC-University of Glasgow Centre for Virus Research, Glasgow, UK. ${ }^{228}$ Liverpool School of Tropical Medicine, Liverpool, UK. ${ }^{229}$ Institute of Microbiology and Infection, University of Birmingham, Birmingham, UK. ${ }^{230}$ Institute of Infection and Global Health, University of Liverpool, Liverpool, UK. ${ }^{231}$ Department of Infectious Diseases, Queen Elizabeth University Hospital, Glasgow, UK. ${ }^{232}$ Virology Reference Department, National Infection Service, Public Health England, London, UK. ${ }^{233}$ Department of Pharmacology, University of Liverpool, Liverpool, UK. ${ }^{234}$ Nuffield Department of Medicine, John Radcliffe Hospital, Oxford, UK. ${ }^{235}$ Translational Gastroenterology Unit, Nuffield Department of Medicine, University of Oxford, Oxford, UK. ${ }^{236}$ Department of Microbiology and Infectious Diseases, Oxford University Hospitals NHS Foundation Trust, John Radcliffe Hospital, Oxford, UK. ${ }^{237}$ Division of Infection and Immunity, University College London, London, UK. ${ }^{238}$ Institute of Infection, Veterinary and Ecological Sciences, University of Liverpool, Liverpool, UK. ${ }^{239} \mathrm{NIHR}$ Health Protection Research Unit in Emerging and Zoonotic Infections, Liverpool, UK. ${ }^{240}$ Centre for Clinical Infection and Diagnostics Research, Department of Infectious Diseases, School of Immunology and Microbial Sciences, King's College London, London, UK. ${ }^{241}$ Department of 
Infectious Diseases, Guy's and St Thomas' NHS Foundation Trust, London, UK. ${ }^{242}$ Institute of Evolutionary Biology, University of Edinburgh, Edinburgh, UK. ${ }^{243}$ Department of Pediatrics and Virology, St Mary's Medical School, Imperial College London, London, UK. ${ }^{244} \mathrm{NHS}$ Greater Glasgow \& Clyde, Glasgow, UK. ${ }^{245}$ The Florey Institute for Host-Pathogen Interactions, Department of Infection, Immunity and Cardiovascular Disease, University of Sheffield, Sheffield, UK. ${ }^{246}$ Walton Centre NHS Foundation Trust, Liverpool, UK. ${ }^{247}$ MRC Centre for Molecular Bacteriology and Infection, Imperial College London, London, UK. ${ }^{248}$ Division of Structural Biology, The Wellcome Centre for Human Genetics, University of Oxford, Oxford, UK. ${ }^{249}$ Blood Borne Virus Unit, Virus Reference Department, National Infection Service, Public Health England, London, UK. ${ }^{250}$ Transfusion Microbiology, National Health Service Blood and Transplant, London, UK. ${ }^{251}$ Department of Medicine, Imperial College London, London, UK. ${ }^{252}$ Department of Infection, Immunity and Cardiovascular Disease, University of Sheffield, Sheffield, UK. ${ }^{253}$ Tropical \& Infectious Disease Unit, Royal Liverpool University Hospital, Liverpool, UK. ${ }^{254}$ Liverpool Clinical Trials Centre, University of Liverpool, Liverpool, UK. ${ }^{255}$ Centre for Health Informatics, Division of Informatics, Imaging and Data Science, School of Health Sciences, Faculty of Biology, Medicine and Health, University of Manchester, Manchester Academic Health Science Centre, Manchester, UK. ${ }^{256}$ Department of Molecular and Clinical Cancer Medicine, University of Liverpool, Liverpool, UK. ${ }^{257}$ Institute of Translational Medicine, University of Liverpool, Liverpool, Merseyside, UK. A full list of members and their affiliations appears in the Supplementary Information.

\section{HGI Consortium (COVID-19 Host Genetics Initiative)}

Andrea Ganna ${ }^{258,259}$, Patrick Sulem ${ }^{260}$, David A. van Heel $^{261}$, Mattia Cordiolii ${ }^{258}$,

Alessandra Renieri ${ }^{32,33}$, Gardar Sveinbjornsson ${ }^{260}$, Mari E. K. Niemi ${ }^{258}$ \&

Alexandre C. Pereira ${ }^{30,31}$

${ }^{258}$ Institute for Molecular Medicine Finland, University of Helsinki, Helsinki, Finland. ${ }^{259}$ Analytic \& Translational Genetics Unit, Massachusetts General Hospital, Harvard Medical School, Boston, MA, USA. ${ }^{260} \mathrm{deCODE}$ genetics/Amgen, Reykjavik, Iceland. ${ }^{261}$ Blizard Institute, Queen Mary University of London, London, UK. A full list of members and their affiliations appears in the Supplementary Information.

\section{3andMe Contributors}

Janie F. Shelton ${ }^{262}$, Anjali J. Shastri ${ }^{262}$, Chelsea $\mathrm{Ye}^{262}$, Catherine H. Weldon ${ }^{262}$, Teresa Filshtein-Sonmez ${ }^{262}$, Daniella Coker ${ }^{262}$, Antony Symons ${ }^{262}$, Jorge Esparza-Gordillo ${ }^{263}$, The 23andMe COVID-19 Team, Stella Aslibekyan ${ }^{262}$ \& Adam Auton ${ }^{262}$

${ }^{262} 23 a n d M e$, Sunnyvale, CA, USA. ${ }^{263}$ Human Genetics-R\&D, GSK Medicines Research Centre Target Sciences-R\&D, GlaxoSmithKline, Stevenage, UK. A full list of members and their affiliations appears in the Supplementary Information.

\section{GEN-COVID Contributors}

Francesca Mari ${ }^{32,33}$, Sergio Daga ${ }^{32}$, Margherita Baldassarri ${ }^{32}$, Elisa Benetti ${ }^{264}$, Simone Furini ${ }^{264}$, Chiara Fallerini ${ }^{32}$, Francesca Fava ${ }^{32,33}$, Floriana Valentino ${ }^{32}$, Gabriella Doddato $^{32}$, Annarita Giliberti ${ }^{32}$, Rossella Tita ${ }^{33}$, Sara Amitrano ${ }^{33}$,

Mirella Bruttini ${ }^{32,33}$, Susanna Croci ${ }^{32}$, Ilaria Meloni ${ }^{32}$, Anna Maria Pinto ${ }^{33}$, Elisa Frullanti ${ }^{32}$, Maria Antonietta Mencarelli ${ }^{33}$, Caterina Lo Rizzo ${ }^{33}$, Francesca Montagnani ${ }^{265}$, Laura Di Sarno $^{32}$, Andrea Tommasi ${ }^{32,33}$, Maria Palmieri ${ }^{32}$, Arianna Emiliozzi ${ }^{265}$,

Massimiliano Fabbiani ${ }^{265}$, Barbara Rossetti ${ }^{265}$, Giacomo Zanelli ${ }^{265}$, Elena Bargagli ${ }^{266}$ Laura Bergantini ${ }^{266}$, Miriana D'Alessandro ${ }^{266}$, Paolo Cameli ${ }^{266}$, David Bennet ${ }^{266}$, Federico Anedda ${ }^{267}$, Simona Marcantonio ${ }^{267}$, Sabino Scolletta ${ }^{267}$, Federico Franchi ${ }^{267}$ Maria Antonietta Mazzei ${ }^{268}$, Susanna Guerrini ${ }^{268}$, Edoardo Conticini ${ }^{269}$, Luca Cantarini ${ }^{269}$, Bruno Frediani ${ }^{269}$, Danilo Tacconi ${ }^{270}$, Chiara Spertilli ${ }^{270}$, Marco Feri ${ }^{271}$, Alice Donati ${ }^{271}$, Raffaele Scala ${ }^{272}$, Luca Guidelli ${ }^{272}$, Genni Spargi ${ }^{273}$, Marta Corridi ${ }^{273}$, Cesira Nencioni ${ }^{274}$, Leonardo $\mathrm{Croci}^{274}$, Gian Piero Caldarelli ${ }^{275}$, Maurizio Spagnesi ${ }^{276}$, Paolo Piacentini ${ }^{276}$ Maria Bandini ${ }^{276}$, Elena Desanctis ${ }^{276}$, Silvia Cappelli ${ }^{276}$, Anna Canaccini ${ }^{277}$, Agnese Verzuri ${ }^{277}$, Valentina Anemoli ${ }^{277}$, Agostino Ognibene ${ }^{278}$, Massimo Vaghi ${ }^{279}$, Antonella D'Arminio Monforte ${ }^{280}$, Esther Merlini ${ }^{280}$, Mario U. Mondelli ${ }^{281,282}$, Stefania Mantovani $^{281}$, Serena Ludovisi ${ }^{281,282}$, Massimo Girardis ${ }^{283}$, Sophie Venturelli ${ }^{283}$, Marco Sita ${ }^{283}$ Andrea Cossarizza ${ }^{284}$, Andrea Antinori ${ }^{285}$, Alessandra Vergori ${ }^{285}$, Stefano Rusconi ${ }^{286,287}$ Matteo Siano ${ }^{287}$, Arianna Gabrieli ${ }^{287}$, Agostino Riva ${ }^{286,287}$, Daniela Francisci ${ }^{288}$, Elisabetta Schiaroli ${ }^{288}$, Pier Giorgio Scotton ${ }^{289}$, Francesca Andretta ${ }^{289}$, Sandro Panese ${ }^{290}$ Renzo Scaggiante ${ }^{291}$, Francesca Gatti ${ }^{291}$, Saverio Giuseppe Parisi ${ }^{292}$, Francesco Castelli ${ }^{293}$, Maria Eugenia Quiros-Roldan ${ }^{293}$, Paola Magro ${ }^{293}$, Isabella Zanella ${ }^{294}$, Matteo Della Monica $^{295}$, Carmelo Piscopo ${ }^{295}$, Mario Capasso ${ }^{296,297,298}$, Roberta Russo ${ }^{296,297}$, Immacolata Andolfo ${ }^{296,297}$, Achille lolascon ${ }^{296,297}$, Giuseppe Fiorentino ${ }^{299}$, Massimo Carella ${ }^{300}$, Marco Castori ${ }^{300}$, Giuseppe Merla ${ }^{300}$, Filippo Aucella ${ }^{301}$, ${\text { Pamela Raggi }{ }^{302} \text {, Carmen Marciano }}^{302}$, Rita Perna ${ }^{302}$, Matteo Bassetti ${ }^{303,304}$, Ántonio Di Biagio $^{304}$, Maurizio Sanguinetti ${ }^{305,306}$, Luca Masucci ${ }^{305,306}$, Serafina Valente ${ }^{307}$, Marco Mandalà ${ }^{308}$, Alessia Giorli ${ }^{308}$, Lorenzo Salerni ${ }^{308}$, Patrizia Zucchi ${ }^{309}$ Pierpaolo Parravicini ${ }^{309}$, Elisabetta Menatti ${ }^{310}$, Stefano Baratti ${ }^{311}$, Tullio Trotta ${ }^{312}$, Ferdinando Giannattasio ${ }^{312}$, Gabriella Coiro ${ }^{312}$, Fabio Lena ${ }^{313}$, Domenico A. Coviello ${ }^{314}$, Cristina Mussini ${ }^{315}$, Giancarlo Bosio ${ }^{316}$, Enrico Martinelli ${ }^{316}$, Sandro Mancarella ${ }^{317}$, Luisa Tavecchia ${ }^{317}$, Lia Crotti ${ }^{318,319}$, Nicola Picchiotti ${ }^{320,321}$, Marco Gori ${ }^{320,322}$, Chiara Gabbi ${ }^{323}$,
Maurizio Sanarico ${ }^{324}$, Stefano Ceri ${ }^{325}$, Pietro Pinoli ${ }^{325}$, Francesco Raimondi ${ }^{326}$ Filippo Biscarini ${ }^{327,328}$ \& Alessandra Stella ${ }^{327,328}$

${ }^{264}$ Department of Medical Biotechnologies, University of Siena, Siena, Italy. ${ }^{265}$ Department of Specialized and Internal Medicine, Tropical and Infectious Diseases Unit, University Hospital of Siena, Siena, Italy. ${ }^{266}$ Unit of Respiratory Diseases and Lung Transplantation, Department of Internal and Specialist Medicine, University of Siena, Siena, Italy. ${ }^{267}$ Department of Emergency and Urgency, Medicine, Surgery and Neurosciences, Unit of Intensive Care Medicine, Siena University Hospital, Siena, Italy. ${ }^{268}$ Department of Medical, Surgical and Neurosciences and Radiological Sciences, Unit of Diagnostic Imaging, University of Siena, Siena, Italy. ${ }^{269}$ Rheumatology Unit, Department of Medicine, Surgery and Neurosciences, University of Siena, Policlinico Le Scotte, Siena, Italy. ${ }^{270}$ Department of Specialized and Internal Medicine, Infectious Diseases Unit, San Donato Hospital Arezzo, Arezzo, Italy. ${ }^{271}$ Department of Emergency, Anesthesia Unit, San Donato Hospital, Arezzo, Italy. ${ }^{272}$ Department of Specialized and Internal Medicine, Pneumology Unit and UTIP, San Donato Hospital, Arezzo, Italy. ${ }^{273}$ Department of Emergency, Anesthesia Unit, Misericordia Hospital, Grosseto, Italy. ${ }^{274}$ Department of Specialized and Internal Medicine, Infectious Diseases Unit, Misericordia Hospital, Grosseto, Italy. ${ }^{275}$ Clinical Chemical Analysis Laboratory, Misericordia Hospital, Grosseto, Italy. ${ }^{276}$ Department of Preventive Medicine, Azienda USL Toscana Sud Est, Arezzo, Italy. ${ }^{277}$ Territorial Scientific Technician Department, Azienda USL Toscana Sud Est, Arezzo, Italy. ${ }^{278} \mathrm{Clinical}$ Chemical Analysis Laboratory, San Donato Hospital, Arezzo, Italy. ${ }^{279} \mathrm{Chirurgia}$ Vascolare, Ospedale Maggiore di Crema, Crema, Italy. ${ }^{280}$ Department of Health Sciences, Clinic of Infectious Diseases, ASST Santi Paolo e Carlo, University of Milan, Milan, Italy. ${ }^{281}$ Division of Infectious Diseases and Immunology, Fondazione IRCCS Policlinico San Matteo, Pavia, Italy. ${ }^{282}$ Department of Internal Medicine and Therapeutics, University of Pavia, Pavia, Italy. ${ }^{283}$ Department of Anesthesia and Intensive Care, University of Modena and Reggio Emilia, Modena, Italy. ${ }^{284}$ Department of Medical and Surgical Sciences for Children and Adults, University of Modena and Reggio Emilia, Modena, Italy. ${ }^{285} \mathrm{HIV} / \mathrm{AIDS}$ Department, National Institute for Infectious Diseases, IRCCS, Lazzaro Spallanzani, Rome, Italy. ${ }^{286}$ III Infectious Diseases Unit, ASST-FBF-Sacco, Milan, Italy. ${ }^{287}$ Department of Biomedical and Clinical Sciences Luigi Sacco, University of Milan, Milan, Italy. ${ }^{288}$ Infectious Diseases Clinic, Department of Medicine University of Perugia, Perugia, Italy. ${ }^{289}$ Department of Infectious Diseases, Treviso Hospital, Treviso, Italy. ${ }^{290} \mathrm{Clinical}$ Infectious Diseases, Mestre Hospital, Venice, Italy. ${ }^{291}$ Infectious Diseases Clinic, Belluno, Italy. ${ }^{292}$ Department of Molecular Medicine, University of Padova, Padua, Italy. ${ }^{293}$ Department of Infectious and Tropical Diseases, University of Brescia, Brescia, Italy. ${ }^{294}$ Department of Molecular and Translational Medicine, University of Brescia, Brescia, Italy. ${ }^{295}$ Medical Genetics and Laboratory of Medical Genetics Unit, A.O.R.N. "Antonio Cardarelli", Naples, Italy. ${ }^{296}$ Department of Molecular Medicine and Medical Biotechnology, University of Naples Federico II, Naples, Italy. ${ }^{297}$ CEINGE Biotecnologie Avanzate, Naples, Italy. ${ }^{298}$ IRCCS SDN, Naples, Italy. ${ }^{299}$ Unit of Respiratory Physiopathology, AORN dei Colli Monaldi Hospital, Naples, Italy. ${ }^{300}$ Division of Medical Genetics, Fondazione IRCCS Casa Sollievo della Sofferenza Hospital, San Giovanni Rotondo, Italy. ${ }^{301}$ Department of Medical Sciences, Fondazione IRCCS Casa Sollievo della Sofferenza Hospital, San Giovanni Rotondo, Italy. ${ }^{302}$ Clinical Trial Office, Fondazione IRCCS Casa Sollievo della Sofferenza Hospital, San Giovanni Rotondo, Italy. ${ }^{303}$ Department of Health Sciences, University of Genova, Genoa, Italy. ${ }^{304}$ Infectious Diseases Clinic, Policlinico San Martino Hospital, IRCCS for Cancer Research Genoa, Genoa, Italy. ${ }^{305}$ Microbiology, Fondazione Policlinico Universitario Agostino Gemelli IRCCS, Catholic University of Medicine, Rome, Italy. ${ }^{306}$ Department of Laboratory Sciences and Infectious Diseases, Fondazione Policlinico Universitario A. Gemelli IRCCS, Rome, Italy. ${ }^{307}$ Department of Cardiovascular Diseases, University of Siena, Siena, Italy. ${ }^{308}$ Otolaryngology Unit, University of Siena, Siena, Italy. ${ }^{309}$ Department of Internal Medicine, ASST Valtellina e Alto Lario, Sondrio, Italy. ${ }^{310}$ Oncologia Medica e Ufficio Flussi, Sondrio, Italy. ${ }^{31}$ Department of Infectious and Tropical Diseases, University of Padova, Padua, Italy. ${ }^{312}$ First Aid Department, Luigi Curto Hospital, Polla, Salerno, Italy. ${ }^{313}$ Local Health Unit, Pharmaceutical Department of Grosseto, Toscana Sud Est Local Health Unit, Grosseto, Italy. ${ }^{314}$ U.O.C. Laboratorio di Genetica Umana, IRCCS Istituto G. Gaslini, Genoa, Italy. ${ }^{315}$ Infectious Diseases Clinics, University of Modena and Reggio Emilia, Modena, Italy. ${ }^{316}$ Department of Respiratory Diseases, Azienda Ospedaliera di Cremona, Cremona, Italy. ${ }^{317}$ U.O.C. Medicina, ASST Nord Milano, Ospedale Bassini, Cinisello Balsamo, Italy. ${ }^{318}$ Istituto Auxologico Italiano, IRCCS, San Luca Hospital, Milan, Italy. ${ }^{319}$ Department of Medicine and Surgery, University of Milano-Bicocca, Milan, Italy. ${ }^{320}$ University of Siena, DIISM-SAILAB, Siena, Italy. ${ }^{321}$ Department of Mathematics, University of Pavia, Pavia, Italy. ${ }^{322}$ University Cote d'Azur, Inria, CNRS, I3S, Maasai, Nice, France. ${ }^{32}$ Independent Medical Scientist, Milan, Italy. ${ }^{324}$ Independent Data Scientist, Milan, Italy. ${ }^{325}$ Department of Electronics, Information and Bioengineering (DEIB), Politecnico di Milano, Milano, Italy. ${ }^{326}$ Scuola Normale Superiore, Pisa, Italy. ${ }^{327} \mathrm{CNR}$-Consiglio Nazionale delle Ricerche, Istituto di Biologia e Biotecnologia Agraria (IBBA), Milano, Italy. ${ }^{328}$ Present address: ERCEA (European Research Council Executive Agency), Bruxelles, Belgium. A full list of members and their affiliations appears in the Supplementary Information.

\section{BRACOVID Contributors}

Alexandre C. Pereira ${ }^{30,31}$, Jose E. Krieger ${ }^{31}$, Emmanuelle Marques ${ }^{31}$ \& Cinthia E. Jannes ${ }^{31}$ 


\section{Methods}

\section{Data reporting}

No statistical methods were used to predetermine sample size. The experiments were not randomized and the investigators were not blinded to allocation during experiments and outcome assessment.

\section{Recruitment of cases}

In total, 2,636 patients who were recruited to the GenOMICC study (https://genomicc.org/) had confirmed COVID-19 according to local clinical testing and were deemed, in the view of the treating clinician, to require continuous cardiorespiratory monitoring. In UK practice this kind of monitoring is undertaken in high-dependency or intensive care units. An additional 135 patients were recruited through ISARIC4C (https://isaric4c.net/)-these individuals had confirmed COVID-19 according to local clinical testing and were deemed to require hospital admission. Both studies were approved by the appropriate research ethics committees (Scotland,15/SS/0110; England, Wales and Northern Ireland,19/WM/0247). Current and previous versions of the study protocol are available at https://genomicc.org/protocol/. All participants gave informed consent.

\section{Genotyping}

DNA was extracted from whole blood using the Nucleon Kit (Cytiva) with the BACC3 protocol. DNA samples were resuspended in $1 \mathrm{ml}$ TE buffer pH 7.5 (10 mM Tris-Cl pH 7.5, 1 mM EDTA pH 8.0). The DNA yield was measured using Qubit and normalized to $50 \mathrm{ng}^{-1} \mathrm{l}^{-1}$ before genotyping.

Genotyping was performed using the Illumina Global Screening Array v.3.0 + multi-disease bead chips (GSAMD-24v3-0-EA) and Infinium chemistry. In summary, this consists of three steps: (1) whole-genome amplification; (2) fragmentation followed by hybridization; and (3) single-base extension and staining. For each of the samples, $4 \mu \mathrm{l}$ of DNA normalized to $50 \mathrm{ng} \mathrm{l}^{-1}$ was used. Each sample was interrogated on the arrays against 730,059 SNPs. The arrays were imaged on an Illumina iScan platform and genotypes were called automatically using GenomeStudio Analysis software v.2.0.3, GSAMD-24v3-0-EA_20034606_ A1.bpm manifest and cluster file provided by the manufacturer.

In 1,667 cases, genotypes and imputed variants were confirmed with Illumina NovaSeq 6000 whole-genome sequencing (WGS). Samples were aligned to the human reference genome hg38 and variant called to GVCF stage on the DRAGEN pipeline (software v.01.011.269.3.2.22, hardware v.01.011.269) at Genomics England. Variants were genotyped with the GATK GenotypeGVCFs tool v.4.1.8.1 ${ }^{50}$, filtered to minimum depth $8 \times\left(95 \%\right.$ sensitivity for heterozygous variant detection ${ }^{51}$,) merged and annotated with allele frequency with bcftools v.1.10.2.

\section{Quality control}

Genotype calls were carefully examined within GenomeStudio using manufacturer's and published ${ }^{52}$ recommendations, after excluding samples with low initial call rate $(<90 \%)$ and reclustering the data thereafter. In brief, $\mathrm{X}$ and $\mathrm{Y}$ marker calls were all visually inspected and curated if necessary, as were those calls of autosomal markers with minor allele frequency (MAF) $>1 \%$ that had a low Gentrain score, cluster separation and an excess or deficit of heterozygous calls. Genotype-based sex determination was performed in GenomeStudio and samples were excluded if they did not match the expectation based on clinical records. Five individuals with XXY genotypes were also detected and excluded for downstream GWAS analyses. Genotypes were exported, in Genome Reference Consortium human build 37 (GRCHb37) and Illumina 'source' strand orientation, using the GenotypeStudio PLINK input report plugin v.2-1-4. A series of filtering steps was then applied using PLINK 1.9, leaving 2,790 individuals and 479,095 variants for further analyses (after exclusion of samples with a call rate of $<95 \%$, selection of variants with call rate of $>99 \%$ and MAF $>1 \%$ and the final selection of samples using a call rate of $>97 \%$ ).

\section{Kinship}

Kinship and ancestry inferences were calculated following the UK Biobank $^{49}$ and 1 Million Veteran Program ${ }^{53}$. First, King $2.1^{54}$ was used to find duplicate individuals who have been recruited by two different routes. The analysis flagged 56 duplicate pairs, from which one was removed according to genotyping quality (GenomeStudio p50GC score and/or individual call rate). This lef a set of 2,734 unique individuals.

Regions of high linkage disquilibrium (LD) defined in the UK Biobank ${ }^{49}$ were excluded from the analysis, as well as SNPs with $\mathrm{MAF}<1 \%$ or missingness $>1 \%$. King 2.1 was used to construct a relationship matrix up to the third degree using the King command --kinship --degree 3 and then the function largest_independent_vertex_set() from the igraph tool http://igraph.sf.net was used to create a first set of unrelated individuals. Principal component analysis (PCA) was conducted with gcta $1.9^{55}$ in the set of unrelated individuals with pruned SNPs using a window of 1,000 markers, a step size of 80 markers and an $r^{2}$ threshold of 0.1. SNPs with large weights in PC1, PC2 or PC3 were removed, keeping at least two-thirds of the number of pruned SNPs to keep as an input for the next round of King 2.1. The second round of King 2.1 was run using the SNPs with low weights in PC1, PC2 and PC3 to avoid overestimating kinship in individuals of non-European ancestry. After this round 2,718 individuals were considered unrelated up to the third degree.

\section{Genetic ancestry}

Unrelated individuals from the 1,000 Genome Project dataset were calculated using the same procedure as described above, and both datasets were merged using the common SNPs. The merged genotyped data was pruned with PLINK using a window of 1,000 markers, a step size of 50 and a $r^{2}$ of 0.05 , leaving 92,017 markers that were used to calculate the 20 first principal components with gcta 1.9. Ancestry for GenOMICC individuals was inferred using ADMIXTURE ${ }^{56}$ populations defined in the 1000 Genomes Project. When one individual had a probability $>80 \%$ of pertaining to one ancestry, then the individual was assigned to this ancestry, otherwise the individual was assigned to 'admixed' ancestry as in the 1 Million Veteran cohort ${ }^{53}$. According to this criterion, there are 1,818 individuals of European ancestry, 190 individuals of African ancestry, 158 individuals of East Asian ancestry, 254 individuals of South Asian ancestry and 301 individuals with admixed ancestry ( 2 or more ancestries).

\section{Imputation}

Genotype files were converted to plus strand and SNPs with HardyWeinberg equilibrium (HWE) $P<10^{-6}$ were removed. Imputation was calculated using the TOPMed reference pane ${ }^{57}$ and results were given in the GRCh38 human reference genome and plus strand. The imputed dataset was filtered for monogenic and low imputation quality score $\left(r^{2}<0.4\right)$ using BCFtools 1.9. To perform GWAS analyses, files in VCF format were further filtered for $r^{2}>0.9$ and converted to BGEN format using QCtools $1.3^{58}$.

UK Biobank imputed variants with imputation score $>0.9$ and overlapping our set of variants $(n=5,981,137)$ were extracted and merged with GenOMICC data into a single BGEN file containing cases and controls using QCtools 1.3.

\section{GWAS}

Related individuals to third degree were removed. In addition, 13 individuals with American ancestry were removed as the sample size provided insufficient power to perform a reliable GWAS analyses for this group. The final dataset includes 2,244 individuals. Using PCA to infer genetic ancestry, there were 1,676 individuals of European ancestry, 149 individuals of East Asian ancestry, 237 individuals of South Asian ancestry and 182 individuals of African ancestry (Extended Data Table 1). If age or deprivation status were missing for some individuals, the value 
was set to the mean of their ancestry. GWAS analyses were performed separately for each ancestry group.

Tests for association between case-control status and allele dosage at individual SNPs were performed by fitting logistic regression models using PLINK $^{59}$. Independent analyses were performed for each ethnic group. All models included sex, age, mean-centred age ${ }^{2}$, deprivation score decile of residential postcode, and the first 10 genomic principal components as covariates.

Genomic principal components were computed on the combined sample of all UK Biobank and GenOMICC participants. Specifically, 456,750 genetic variants were identified that were shared between the variants contained in the called genotypes in the GenOMICC dataset and imputed UK Biobank genotypes, which had an imputation info score $>0.95$ and $\mathrm{MAF}>1 \%$. After merging genotypes at these variants, variants were removed that had $\mathrm{MAF}<2.5 \%$, a missingness rate $>1.5 \%$, showed departure from HWE $P<10^{-50}$ or were within previously identified regions of high linkage disequilibrium within the UK Biobank. After LD pruning of the remaining variants to a maximum $r^{2}$ of 0.01 based on a 1,000-variant window moving in 50 variants steps, using the PLINK indep-pairwise command and yielding 13,782 SNPs, the leading 20 genomic principal components were computed using FlashPCA2 ${ }^{60}$.

GWAS results for individuals of European ancestry were filtered for $\mathrm{MAF}>0.01, \mathrm{HWE} P>10^{-50}$ and genotyping rate $>0.99$. An extra filter was added to avoid bias for using a different genotyping method and imputation panel between controls and cases. This could not be controlled for using regression because all cases and all controls were genotyped using different methods. MAFs for each ancestry were compared between UK Biobank European controls and gnomAD hg38 non-Finnish European individuals downloaded in August $2020^{61}$. SNPs were removed from the GWAS results following two rules: (1) In SNPs with MAF > 10\% in gnomAD, an absolute difference in $\mathrm{MAF}$ of $5 \%$ between gnomAD and UK Biobank controls; (2) in SNPs with MAF $<10 \%$ in gnomAD, a difference in MAF of $>25 \%$ between UK Biobank controls and gnomAD. GWAS analyses of individuals of non-European ancestries were filtered for a MAF in UK Biobank controls corresponding to the same ancestry $>5 \%$ and then for the SNPs that passed quality control in the European GWAS. To calculate differences between UK Biobank European individuals and gnomAD allele frequencies, gnomAD allele frequencies for individuals of non-Finnish European descent were used, as European UK Biobank controls are mainly non-Finnish.

Filtered GWAS analyses for each ancestry, containing a total of around 4.7 million SNPs, were combined in a trans-ethnic meta-analysis using METAL ${ }^{62}$ standard error mode and controlling for population stratification (genomic control on). The nearest genes were defined using the SNP2GENE function in FUMA v.1.3.6. $6^{63}$, using LD $r^{2}>0.6$ and UK Biobank release 2 reference panel.

A sex-specific GWAS within European individuals was performed using 1,180 cases of unrelated men and 496 cases of unrelated women and 5 UK Biobank random control individuals matched by sex and ancestry for each case. Tests for association between case-control status and allele dosage at individual SNPs were performed by fitting a logistic regression model with PLINK. Age, mean age squared, deprivation decile of residential postcode and the first 10 principal components were added as covariates in the models.

Deprivation score. The UK Data Service provides measures of deprivation based on census data and generated per postcode. The latest version of the deprivation scores were published in 2017 and are based on the 2011 census. As only partial postcodes were available for most samples we were unable to use these indices directly. However, we generated an approximation to the scores by calculating an average weighted by population count across the top-level postcode areas.

The initial input file was part of the aggregated census data identified by in the 2011 Census aggregate $e^{85}$ and the postcode data were downloaded from http://s3-eu-west-1.amazonaws.com/statistics.
digitalresources.jisc.ac.uk/dkan/files/Postcode_Counts_and_Deprivation Ranks/postcodes.zip.

Population count and deprivation score for each published postcode were extracted and the weighted average score was calculated for each top-level postcode. We further categorized each top-level postcode score into decile and quintile bins for more coarse-grained analyses.

WGS. WGS gVCF files were obtained for the 1,667 individuals for whom we had WGS data. Variants overlapping the positions of the imputed variants were called using GATk and variants with depth $<8 \times$ (the minimum depth for which $95 \%$ coverage can be expected) were filtered. Individual VCF files were joined in a multi-sample VCF file for comparison with imputed variants. We used 1,613 of the 1,667 individuals in the final GWAS. Samples were filtered and variants were annotated using bcftools 1.9. VCF files obtained from imputation were processed in an identical manner. Alternative allele frequencies were calculated with PLINK $2.0^{64}$ for both WGS and imputed data.

\section{Controls}

UK Biobank. UK Biobank participants were considered as potential controls if they were not identified by the UK Biobank as outliers based on either genotyping missingness rate or heterogeneity, and their sex inferred from the genotypes matched their self-reported sex. For these individuals, information on sex (UKBID 31), age, ancestry and residential postcode deprivation score decile was computed. Specifically, age was computed as age on 1 April 2020 based on the participant's birth month (UKBID 34) and year (UKBID 52). The first part of the residential postcode of participants was computed based on the participant's home location (UKBID 22702 and 22704) and mapped to a deprivation score decile as described above for GenOMICC participants. Ancestry was inferred as described above for GenOMICC participants.

After excluding participants who had received PCR tests for COVID19 , based on information downloaded from the UK Biobank in August 2020, five individuals with matching inferred ancestry were sampled for each GenOMICC participant as controls. After sampling each control, individuals related up to the third degree were removed from the pool of potential further controls. An additional analysis with more stringent matching on individual characteristics was also performed (Supplementary Information, 'Matched controls').

The 100,000 Genomes Project. Following ethical approval (14/EE/1112 and $13 / \mathrm{EE} / 032$ ), consenting participants from the 100,000 Genomes Project with a broad range of rare diseases, cancers and infection were enrolled by 13 regional NHS Genomic Medicine Centres across England and in Northern Ireland, Scotland and Wales and whole blood was drawn for DNA extraction. After quality assurance, WGS at 125 or 150 base pairs was performed by Illumina Laboratory Services on either Hiseq 2500 or Hiseq X sequencers in the Genomics England Sequencing Centre, followed by detection of small variants (single-nucleotide variants and small insertions or deletions) using Starling.

Tests for association between cases and control status were performed by running mixed model association tests using SAIGE (v.0.39). In total, 1,675 individuals from the GenOMICC study and 45,875 unrelated participants of European ancestry were included. Genomic principal components were calculated for the combined dataset of GenOMICC participants and WGS data from the 100,000 Genomes Project. PCA was performed with GCTA software using approximately 30,000 SNPs selected with MAF $>0.005$ and after LD pruning $\left(r^{2}<0.1\right.$ with a window size of $500 \mathrm{~kb}$ ). Fitting of the null logistic mixed model was performed using the SNPs used for PCA and included age, sex, squared age, age $\times$ sex and the first 20 genomic principal components as covariates.

Tests for association using SAIGE were performed after filtering variants in the WGS dataset for genotype quality and MAF $\geq 0.05$. GWAS-specific quality filtering was performed to include variants 
with minor allele count $\geq 20$ for each phenotype, differential missingness between cases and controls $\left(P<1 \times 10^{-5}\right)$ and departure from HWE $\left(P<1 \times 10^{-5}\right)$.

Generation Scotland. The Generation Scotland: Scottish Family Health Study is a population-based cohort of 24,084 participants sampled from five regional centres across Scotland ${ }^{65}$. A large subset of participants were genotyped using either Illumina HumanOmniExpressExome-8v1_A or v1-2, and 20,032 passed quality control criteria that have previously been described ${ }^{66,67}$. Genotype imputation using the TOPMed reference panel was recently performed (freeze $5 \mathrm{~b}$ ) using Minimac4 v.1.0 on the University of Michigan server (https://imputationserver.sph. umich.edu) ${ }^{68}$. Imputation data from 7,689 unrelated (genomic sharing identical-by-descent estimated to be $<5 \%$ using PLINK 1.9) participants were used as control genotypes in a GWAS using GenOMICC cases of European ancestry, for quality control purpose of associated variants. GWAS was performed in a logistic regression framework implemented in the gIm function of PLINK 2 (https://www.cog-genomics.org/ plink/2.0/), adjusting for age, sex and the first 10 principal components of European ancestry. These coordinates were obtained from projection to the principal component space of the European population samples of the 1000 Genomes Project using KING v.2.2.5 $5^{54}$ and a LD-pruned subset of target genotyped markers that passed quality control and intersecting with the reference populations.

\section{Validation}

Clumped hits in discovery GWAS analyses were validated using controls from Generation Scotland and the 100,000 Genomes Project. To consider a hit validated, the direction of effect should be the same in all three GWAS datasets and the $P$ value in both Generation Scotland and the 100,000 Genomes Project had to be $P<0.05 / n_{\text {validations }}$, where $n_{\text {validations }}$ is the number of significant independent loci in our analysis at the discovery threshold of $P<5 \times 10^{-8}$.

\section{Replication}

Loci in GenOMICC individuals of European ancestry were defined using the clump function of PLINK $1.9^{64}$ and clumping parameters $r^{2}=0.1$, $P=5 \times 10^{-8}$ and $P_{2}=0.01$; distance to the nearest gene was calculated using ENSEMBL GRCh37 gene annotation.

No GWAS has been reported of critical illness or mortality in COVID19. As a surrogate, to provide some replication for our findings, replication analyses were performed using HGI build 37, version 2 (July 2020) B2 (hospitalized patients with COVID-19 versus the population) GWAS. Summary statistics were used from the full analysis, including all cohorts and GWAS without UK Biobank, to avoid sample overlap. The replication $P$ value was set to $6.25 \times 10^{-4}(0.05 / 8$, where 8 is the number of loci significant in the discovery analysis).

\section{Genome-wide meta-analysis}

Meta-analysis of the GenOMICC, HGI and 23andMe datasets was performed using fixed-effect inverse-variance meta-analysis in METAL ${ }^{62}$, with correction for genomic control on. The 23andMe study comprises cases and controls from an European genetic ancestry group. The HGI B2 analysis is a trans-ancestry meta-analysis, with the great majority of cases being multi-ethnic European (European and Finnish), with 238 cases of non-European ancestry (176 individuals of admixed American descent from the BRACOVID study and 62 individuals of South Asian ancestry from the GNH study).

\section{Post-GWAS analyses}

TWAS and Meta-TWAS. We performed transcriptome-wide association using the MetaXcan framework ${ }^{23}$ and the GTEx v.8 eQTL MASHR-M models (http://predictdb.org/). To increase SNP coverage to perform TWAS, GWAS summary statistics for European ancestry were first imputed using the fizi ${ }^{69}$ impute function (https://github.com/bogdanlab/fizi), the European population from the 1000 Genomes Project as LD reference and $30 \%$ as minimum proportion of SNPs for a region (--min-prop 0.3). Then, imputed GWAS results were harmonized, lifted over to hg38 and linked to the 1000 Genomes Project reference panel using GWAS tools (https://github.com/hakyimlab/summary-gwas-imputation/ wiki/GWAS-Harmonization-And-Imputation).

Imputed and harmonized GWAS summary statistics were used to perform TWAS for whole-blood samples (Supplementary Fig. 16) and lung tissues (Fig. 2) in GTEx v.8 with the S-PrediXcan function. Resulting $P$ values were corrected using the Bonferroni correction to find significant gene associations. To overcome the limitations of sample size in GTEx v.8 lung tissues and whole-blood samples, we performed a meta-TWAS prioritizing genes with small $P$ values in these tissues and using GTEx v.8 gene expression in all tissues and S-Multixcan ${ }^{70}$.

Mendelian randomization. Mendelian randomization ${ }^{19}$ based on two-sample summary data was performed using the results of GenOMICC and GTEx v. $7^{71}$ (using SMR/HEIDI pre-prepared data from https:// cnsgenomics.com/software/smr/\#DataResource), with Generation Scotland ${ }^{65,72}$ forming a LD reference. GenOMICC results from individuals of European ancestry were used as the outcome; and GTEx (v.7) whole-blood expression results as the exposure. Additional data pertaining to GTEx v.7 were downloaded from GTEx: https://gtexportal.org/ (accessed 20 February, 5 April and 4 July 2020), and SMR/ HEIDI from https://cnsgenomics.com/software/smr/ (accessed 3July 2020). Analyses were conducted using Python v.3.7.3 and SMR/HEIDI v.1.03 (plots were made using SMR/HEIDI v.0.711). An LD reference was created using data from the population-based Generation Scotland cohort (used with permission; described previously ${ }^{67}$ ): from a random set of 5,000 individuals, using PLINK v.1.9 (www.cog-genomics.org/ plink/1.9/), a set of individuals with a genomic relatedness cut-off of $<0.01$ was extracted; 2,778 individuals remained in the final set. All data used for the SMR/HEIDI analyses were limited to autosomal biallelic SNPs: 4,264,462 variants remained in the final merged dataset.

Significant (as per GTEx v.7; nominal $P$ value below the nominal $P$-value threshold) local (distance to transcriptional start site $<1 \mathrm{Mb}$ ) eQTLs from GTEx v.7 whole-blood samples for protein-coding genes (as per GENCODE v.19) with a MAF > 0.01 (GTEx v.7 and GenOMICC) were considered to be potential instrumental variables. Per variant, we first selected the Ensembl gene ID with which the eQTL was most strongly associated followed by selecting the variant with which each Ensembl gene ID was most strongly associated. Instruments were available for 4,614 unique Ensembl gene IDs.

Results were assessed based on a list of genes selected a priori as of interest (Supplementary Table 3), and together as a whole. Replication of Bonferroni-corrected significant results was attempted for the results of COVID-19 HGI (https://www.covid19hg.org/) with the UK Biobank excluded (2 July 2020 data release) using the eQTLgen expression dataset ${ }^{20}$. Hospitalized patients with COVID-19 versus population (ANA_B2_V2) was selected as the phenotype most similar to our own, and therefore the most appropriate to use as a replication cohort.

To further validate the analyses above, generalized summary-data Mendelian randomization (GSMR) ${ }^{73}$ was performed using exposure data from https://www.eqtlgen.org/index.html (accessed 26 October 2020$)^{20}$ and the publicly available GenOMICC European data for TYK2 and IFNAR2 (Supplementary Fig. 15). GSMR was performed using GCTA v.1.92.1 beta6 Linux. Pleiotropic SNPs were filtered using HEIDI-outlier test (threshold $=0.01$ ) and instrument SNPs were selected at a genome-wide significance level $\left(P_{\mathrm{eQTL}}<5 \times 10^{-8}\right)$ using LD clumping ( $\mathrm{LD} r^{2}$ threshold $=0.05$ and window size $=1 \mathrm{Mb}$ ). The imputed genotypes for 50,000 unrelated individuals (based on SNP-derived genomic relatedness $<0.05$ using SNPs from HapMap 3) from the UK Biobank were used as the LD reference for clumping. GSMR accounts for remaining LD not removed by LD clumping. 
Genomic region plots. Genomic region plots were created using https://github.com/Geeketics/LocusZooms (Supplementary Figs. 5, 6).

Gene-level and pathway analyses. The gene-level burden of significance in the results of the European ancestry group was calculated using MAGMA v.1.08 ${ }^{74}$ (Supplementary Fig. 17). SNPs were annotated to genes by mapping based on genomic location. SNPs were assigned to a gene if the SNPs location was within $5 \mathrm{~kb}$ up-or down-stream of the gene region (defined as the transcription start site to transcription stop site). The MAGMA SNP-wise mean method was applied, which utilizes the sum of squared SNP Z-statistics as the test statistic. The European reference panel of the 1000 Genomes Project was used to estimate LD between SNPs.

Auxiliary files were downloaded from https://ctg.cncr.nl/software/ magma on 1 September 2020. Gene location files for protein-coding genes were obtained from NCBI (ftp.ncbi.nlm.nih.gov) on 29 April 2015 (gene/DATA/GENE_INFO/Mammalia/Homo_sapiens.gene_info. gz) and 25 May 2016 (genomes/Homo_sapiens/ARCHIVE/ANNOTATION_RELEASE.105/mapview/seq_gene.md.gz).

The reference data files used to estimate $L D$ are derived from Phase 3 of the 1000 Genomes Project.

Competitive gene set enrichment analysis was conducted in MAGMA using a regression model that accounts for gene-gene correlations to reduce bias resulting from clustering of functionally similar genes on the genome ${ }^{74}$. Gene sets were queried from the databases KEGG 2019, Reactome 2016, GO Biological Process 2018, Biocarta 2016 and WikiPathways 2019. The Benjamini-Hochberg procedure was used to control false-discovery rate $(<0.05)$.

MAIC. To put these results in the context of existing biological data about host genes in SARS-CoV-2 replication and response, we performed MAIC ${ }^{24}$ analysis, which integrates gene-level results from the GenOMICC metaTWAS and an existing systematic review of host factors implicated in SARS-CoV-2 viral replication and host response in COVID-19.

We developed MAIC to evaluate and integrate gene-level data from diverse sources ${ }^{24}$. Multiple in vitro and in vivo studies have identified key host genes that either directly interact with SARS-CoV-2, or define the host response to SARS-CoV-2. We have previously conducted a systematic review of these studies ${ }^{45}$. To put the new associations from this GWAS into context, we performed a data-driven meta-analysis of gene-level results combined with pre-existing biological data using MAIC $^{24}$.

In brief, MAIC aggregates both ranked and unranked lists and performs better than other methods, particularly when presented with heterogeneous source data. The input to MAIC is a list of named genes. MAIC assigns a score to each gene according to how many source datasets have reported that gene, and then creates a data-driven weighting for each data source (usually an individual experiment) based on the scores of the genes that are highly ranked on that list. This procedure is performed iteratively until the scores and weightings converge on stable values. To prevent a single type of experiment from unduly biasing the results, input gene lists are assigned to categories, and a rule applied that only one weighting from each category can contribute to the score for any given gene.

Tissue and functional genomic enrichment. We downloaded the mean gene expression data summarized from RNA sequencing by the GTEx Project (https://gtexportal.org/). The GTEx v.7 dataset contains gene expression data of 19,791 genes in 48 human tissues. Gene expression values were normalized to numbers of transcripts per million reads. To measure the expression specificity of each gene in each tissue, each gene expression specificity was defined as the proportion of its expression in each tissue among all the tissues - that is, a value ranging between 0 and 1 . SNPs within the $10 \%$ most specifically expressed genes in each tissue were annotated for subsequent testing of heritability enrichment. For functional genomic enrichment analysis, we considered the inbuilt primary functional annotations v.2.2 provided in the Idsc software (https://alkesgroup.broadinstitute.org/LDSCORE/) to annotated the SNPs.

With the annotated SNPs, we used stratified LD score regression ${ }^{75}$ to test whether any human tissue or specific functional genomic feature is associated with severe COVID-19. Our GWAS summary statistics were harmonized by the munge_sumstats.py procedure in Idsc. LD scores of HapMap3 SNPs (MHC region excluded) for gene annotations in each tissue were computed using a 1-cM window. The enrichment score was defined as the proportion of heritability captured by the annotated SNPs divided by the proportion of annotated SNPs.

Genetic correlations. We applied both the LD score regression ${ }^{76}$ and high-definition likelihood ${ }^{25}$ methods to evaluate the genetic correlations between severe COVID-19 and 818 GWAS-analysed phenotypes stored on LD-Hub ${ }^{77}$. GWAS summary statistics were harmonized by the munge_sumstats.py procedure in the Idsc software. In the high-definition likelihood analysis, we estimated the SNP-based narrow-sense heritability for each phenotype, and for the 818 complex traits GWAS analyses, those with SNPs with less than $90 \%$ overlap with the high-definition likelihood reference panel were removed.

\section{Genome build}

Results are presented using Genome Reference Consortium human build 37. Imputed genotypes and WGS data were lifted over from Genome Reference Consortium Human Build 38 using Picard liftoverVCF mode from GATK 4.0, which is based on the UCSC liftover tool (chain file obtained from ftp://ftp.ensembl.org/pub/assembly_mapping/homo_sapiens/GRCh38_to_GRCh37.chain.gz) ${ }^{78}$.

\section{Reporting summary}

Further information on research design is available in the Nature Research Reporting Summary linked to this paper.

\section{Data availability}

Full summary-level data that support the findings of this study are available from https://genomicc.org/data. Individual-level data can be analysed by qualified researchers in the ISARIC4C/GenOMICC data analysis platform by application at https://genomicc.org/data. The full GWAS summary statistics for the 23andMe discovery dataset are available through 23andMe to qualified researchers under an agreement with 23andMe that protects the privacy of the 23andMe participants. More information and access to the data are provided at https:// research.23andMe.com/dataset-access/.

50. McKenna, A. et al. The Genome Analysis Toolkit: a MapReduce framework for analyzing next-generation DNA sequencing data. Genome Res. 20, 1297-1303 (2010).

51. Meynert, A. M., Ansari, M., FitzPatrick, D. R. \& Taylor, M. S. Variant detection sensitivity and biases in whole genome and exome sequencing. BMC Bioinformatics 15, 247 (2014).

52. Guo, Y. et al. Illumina human exome genotyping array clustering and quality control. Nat Protoc. 9, 2643-2662 (2014)

53. Gaziano, J. M. et al. Million Veteran Program: a mega-biobank to study genetic influences on health and disease. J. Clin. Epidemiol. 70, 214-223 (2016).

54. Manichaikul, A. et al. Robust relationship inference in genome-wide association studies. Bioinformatics 26, 2867-2873 (2010).

55. Yang, J., Lee, S. H., Goddard, M. E. \& Visscher, P. M. GCTA: a tool for genome-wide complex trait analysis. Am. J. Hum. Genet. 88, 76-82 (2011).

56. Alexander, D. H. \& Lange, K. Enhancements to the ADMIXTURE algorithm for individual ancestry estimation. BMC Bioinformatics 12, 246 (2011).

57. Taliun, D. et al. Sequencing of 53,831 diverse genomes from the NHLBI TOPMed Program. Preprint at https://doi.org/10.1101/563866 (2019).

58. Wigginton, J. E., Cutler, D. J. \& Abecasis, G. R. A note on exact tests of Hardy-Weinberg equilibrium. Am. J. Hum. Genet. 76, 887-893 (2005).

59. Chang, C. C. et al. Second-generation PLINK: rising to the challenge of larger and richer datasets. Gigascience 4, 7 (2015). 
60. Abraham, G., Qiu, Y. \& Inouye, M. FlashPCA2: principal component analysis of Biobank-scale genotype datasets. Bioinformatics 33, 2776-2778 (2017).

61. Karczewski, K. J. et al. The mutational constraint spectrum quantified from variation in 141,456 humans. Nature 581, 434-443 (2020).

62. Willer, C. J., Li, Y. \& Abecasis, G. R. METAL: fast and efficient meta-analysis of genomewide association scans. Bioinformatics 26, 2190-2191 (2010).

63. Watanabe, K., Taskesen, E., van Bochoven, A. \& Posthuma, D. Functional mapping and annotation of genetic associations with FUMA. Nat. Commun. 8, 1826 (2017).

64. Purcell, S. et al. PLINK: a tool set for whole-genome association and population-based linkage analyses. Am. J. Hum. Genet. 81, 559-575 (2007).

65. Smith, B. H. et al. Cohort Profile: Generation Scotland: Scottish Family Health Study (GS:SFHS). The study, its participants and their potential for genetic research on health and illness. Int. J. Epidemiol. 42, 689-700 (2013).

66. Amador, C. et al. Recent genomic heritage in Scotland. BMC Genomics 16, 437 (2015).

67. Nagy, R. et al. Exploration of haplotype research consortium imputation for genome-wide association studies in 20,032 Generation Scotland participants. Genome Med. 9, 23 (2017).

68. Das, S. et al. Next-generation genotype imputation service and methods. Nat. Genet. 48 , 1284-1287 (2016).

69. Pasaniuc, B. et al. Fast and accurate imputation of summary statistics enhances evidence of functional enrichment. Bioinformatics 30, 2906-2914 (2014).

70. Barbeira, A. N. et al. Integrating predicted transcriptome from multiple tissues improves association detection. PLoS Genet. 15, e1007889 (2019).

71. GTEx Consortium. Genetic effects on gene expression across human tissues. Nature 550, 204-213 (2017).

72. Smith, B. H. et al. Generation Scotland: the Scottish Family Health Study; a new resource for researching genes and heritability. BMC Med. Genet. 7, 74 (2006).

73. Zhu, Z. et al. Causal associations between risk factors and common diseases inferred from GWAS summary data. Nat. Commun. 9, 224 (2018).

74. de Leeuw, C. A., Mooij, J. M., Heskes, T. \& Posthuma, D. MAGMA: generalized gene-set analysis of GWAS data. PLOS Comput. Biol. 11, e1004219 (2015).

75. Finucane, H. K. et al. Partitioning heritability by functional annotation using genome-wide association summary statistics. Nat. Genet. 47, 1228-1235 (2015).

76. Bulik-Sullivan, B. et al. An atlas of genetic correlations across human diseases and traits. Nat. Genet. 47, 1236-1241 (2015).

77. Zheng, J. et al. LD Hub: a centralized database and web interface to perform LD score regression that maximizes the potential of summary level GWAS data for SNP heritability and genetic correlation analysis. Bioinformatics 33, 272-279 (2017).

78. Hinrichs, A. S. et al. The UCSC genome browser database: update 2006. Nucleic Acids Res. 34, D590-D598 (2006).

79. Sun, J. et al. Comparative transcriptome analysis reveals the intensive early-stage responses of host cells to SARS-CoV-2 infection. Preprint at https://doi.org/10.1101/ 2020.04.30.071274 (2020).

80. Rosa, B. A. et al. IFN signaling and neutrophil degranulation transcriptional signatures are induced during SARS-CoV-2 infection. Preprint at https://doi.org/10.1101/ 2020.08.06.239798 (2020).

81. Zhang, J.-Y. et al. Single-cell landscape of immunological responses in patients with COVID-19. Nat. Immunol. 21, 1107-1118 (2020).

82. Mick, E. et al. Upper airway gene expression reveals suppressed immune responses to SARS-CoV-2 compared with other respiratory viruses. Nat. Commun. 11, 5854 (2020).

83. Wei, J. et al. Genome-wide CRISPR screen reveals host genes that regulate SARS-CoV-2 infection. Preprint at https://doi.org/10.1101/2020.06.16.155101(2020).

84. Heaton, B. E. et al. SRSF protein kinases 1 and 2 are essential host factors for human coronaviruses including SARS-CoV-2. Preprint at https://doi.org/10.1101/ 2020.08.14.251207 (2020).

85. UK Data Service. 2011 Census Aggregate Data. https://doi.org/10.5257/census/ aggregate-2011-2 (Office for National Statistics, National Records of Scotland, Northern Ireland Statistics and Research Agency, 2017).

Acknowledgements We thank the patients and their loved ones who volunteered to contribute to this study at one of the most difficult times in their lives, and the research staff in every intensive care unit who recruited patients at personal risk during the most extreme conditions that we have ever witnessed in UK hospitals; R. Coll for advice on the interpretation of these results; J. Zheng for sharing the harmonized GWAS summary statistics used in LD-Hub; and P. McLaren for substantive improvements to the manuscript and analysis. GenOMICC was funded by Sepsis Research (the Fiona Elizabeth Agnew Trust), the Intensive Care Society, a Wellcome-Beit Prize award to J.K.B. (Wellcome Trust 103258/Z/13/A) and a BBSRC Institute
Program Support Grant to the Roslin Institute (BBS/E/D/20002172, BBS/E/D/10002070 and $\mathrm{BBS} / \mathrm{E} / \mathrm{D} / 30002275)$. WGS was done in partnership with Genomics England and was funded by UK Department of Health and Social Care, UKRI and LifeArc. ISARIC4C is supported by grants from the Medical Research Council (grant MC_PC_19059), the National Institute for Health Research (NIHR) (award CO-CIN-O1) and by the NIHR Health Protection Research Unit (HPRU) in Emerging and Zoonotic Infections at University of Liverpool in partnership with Public Health England (PHE), in collaboration with Liverpool School of Tropical Medicine and the University of Oxford (award 200907), NIHR HPRU in Respiratory Infections at Imperial College London with PHE (award 200927), Wellcome Trust and Department for International Development (215091/Z/18/Z), and the Bill and Melinda Gates Foundation (OPP1209135), and Liverpool Experimental Cancer Medicine Centre (grant reference: C18616/A25153), NIHR Biomedical Research Centre at Imperial College London (IS-BRC-1215-20013), EU Platform for European Preparedness Against (Re-)emerging Epidemics (PREPARE) (FP7 project 6025250 and NIHR Clinical Research Network provide infrastructure support for this research. P.J.M.O. is supported by a NIHR Senior Investigator Award (award 201385). H.M. was supported by the NIHR BRC at University College London Hospitals. The Health Research Board of Ireland (Clinical Trial Network Award 2014-12) funded the collection of samples in Ireland. This research has been conducted using the UK Biobank Resource under project 788. Generation Scotland received core support from the Chief Scientist Office of the Scottish Government Health Directorates (CZD/16/6) and the Scottish Funding Council (HRO3006) and is currently supported by the Wellcome Trust (216767/Z/19/Z). Genotyping of the Generation Scotland: Scottish Family Health Study (GS:SFHS) samples was carried out by the Genetics Core Laboratory at the Edinburgh Clinical Research Facility, University of Edinburgh, Scotland and was funded by the Medical Research Council UK and the Wellcome Trust (Wellcome Trust Strategic Award STratifying Resilience and Depression Longitudinally (STRADL) reference 104036/Z/14/Z). Genomics England and the 100,000 Genomes Project was funded by the National Institute for Health Research, the Wellcome Trust, the Medical Research Council, Cancer Research UK, the Department of Health and Social Care and NHS England. M. Caulfield is an NIHR Senior Investigator. This work is part of the portfolio of translational research at the NIHR Biomedical Research Centre at Barts and Cambridge. Research performed at the Human Genetics Unit was funded by the MRC (MC_UU_00007/10, MC_UU_00007/15). L.K. was supported by an RCUK Innovation Fellowship from the National Productivity Investment Fund (MR/R026408/1). A.D.B. acknowledges funding from the Wellcome Trust PhD training fellowship for clinicians (204979/Z/16/Z), and the Edinburgh Clinical Academic Track (ECAT) programme. We acknowledge support from the MRC Human Genetics Unit programme grant 'Quantitative traits in health and disease' (U. MC_UU_00007/10). A.T. acknowledges funding from MRC research grant MR/P015514/1, and HDR-UK award HDR-9004 and HDR-9003. We acknowledge the National Institute of Healthcare Research Clinical Research Network (NIHR CRN) and the Chief Scientist Office (Scotland), who facilitated recruitment into research studies in NHS hospitals, and the global ISARIC and InFACT consortia. The views expressed are those of the authors and not necessarily those of the DHSC, DID, NIHR, MRC, Wellcome Trust or PHE. The views expressed here are purely those of the authors and may not in any circumstances be regarded as stating an official position of the European Commission.

Author contributions J.K., P.K., C. Hinds, P.H., A.N., D. Maslove, L.L., D. McAuley, H.M., T.W., C.P.P. and J.K.B. contributed to study design. S.C., J.F., F.G., W.O., S.K., A.F., K. Rowan, L. Murphy, P.J.M.O., M.G.S., A.L. and J.K.B. contributed to study coordination. N.W., A.F. and L. Murphy contributed to laboratory work. E.P.-C., S.C., L.K., A.D.B., K. Rawlik, D.P., S.W., N.P., M.H.F., J.F., A. Richmond, E.G., D.H., B.W., Y.W., A.M., A.K., L. Moutsianas, Z.Y., R.Z., C.Z., G.G., B.S., M.Z., C. Haley, J.Y., X.S., C.P.P., A.T., K. Rowan, A.L., V.V., J.F.W. and J.K.B. contributed to data analysis. S.C., C.D.R., D.J.P., C. Hayward, C.S., M.S.-H., L.T., A.H., S.C.M., A.C.P., A. Renieri, M.C., R.S. and J.K.B. contributed to the recruitment of cases and controls. S.C., C.D.R., R.B., J.M. and J.K.B. contributed to the interpretation of the findings. E.P.-C., S.C,. L.K., A.D.B., K. Rawlik, C.D.R., R.B., J.M., C.P.P., K. Rowan, V.V., J.F.W. and J.K.B. contributed to manuscript preparation. J.K.B. conceived the study and wrote the first draft of the manuscript. All authors approved the final version of the manuscript. Recruiting sites of the GenOMICC Consortium are listed in descending order of the number of patients recruited per site.

Competing interests The authors declare no competing interests.

\section{Additional information}

Supplementary information The online version contains supplementary material available at https://doi.org/10.1038/s41586-020-03065-y.

Correspondence and requests for materials should be addressed to J.K.B.

Peer review information Nature thanks Paul McLaren and the other, anonymous, reviewer(s)

for their contribution to the peer review of this work. Peer reviewer reports are available. Reprints and permissions information is available at http://www.nature.com/reprints. 
QQ Plot gcc.eur

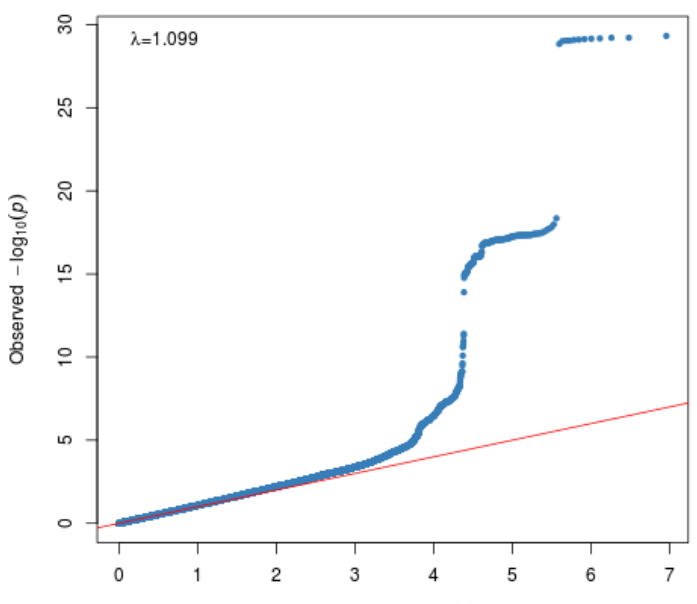

a.

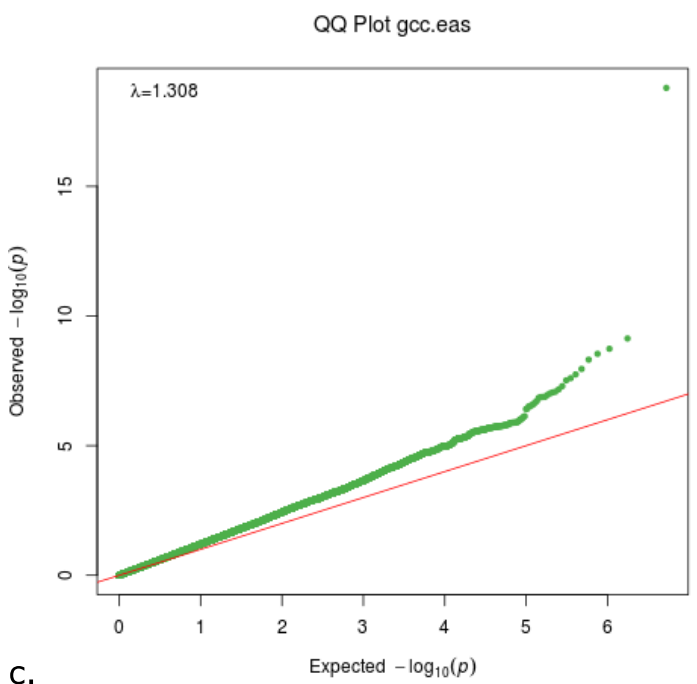

c.

QQ Plot gcc.te.meta

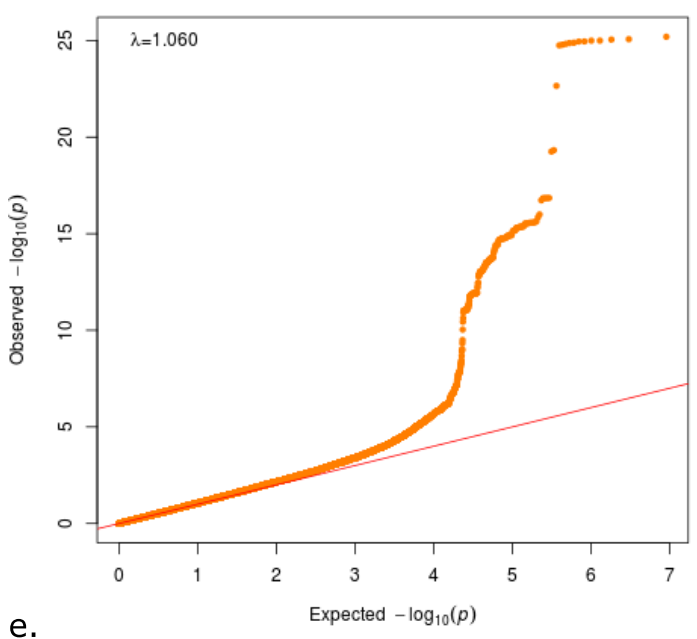

Extended Data Fig. $1 \mid Q Q$ plots. a-f, Raw (unncorrected) $P$ values are shown for each ancestry group in GenOMICC (European (a; gcc.eur); African (b; gcc. afr); East Asian (c; gcc.eas); South Asian (d; gcc.sas)) as well as a trans-ethnic meta-analysis (gcc.te.meta) and a meta-analysis comprising GenOMICC, HGI
QQ Plot gcc.afr
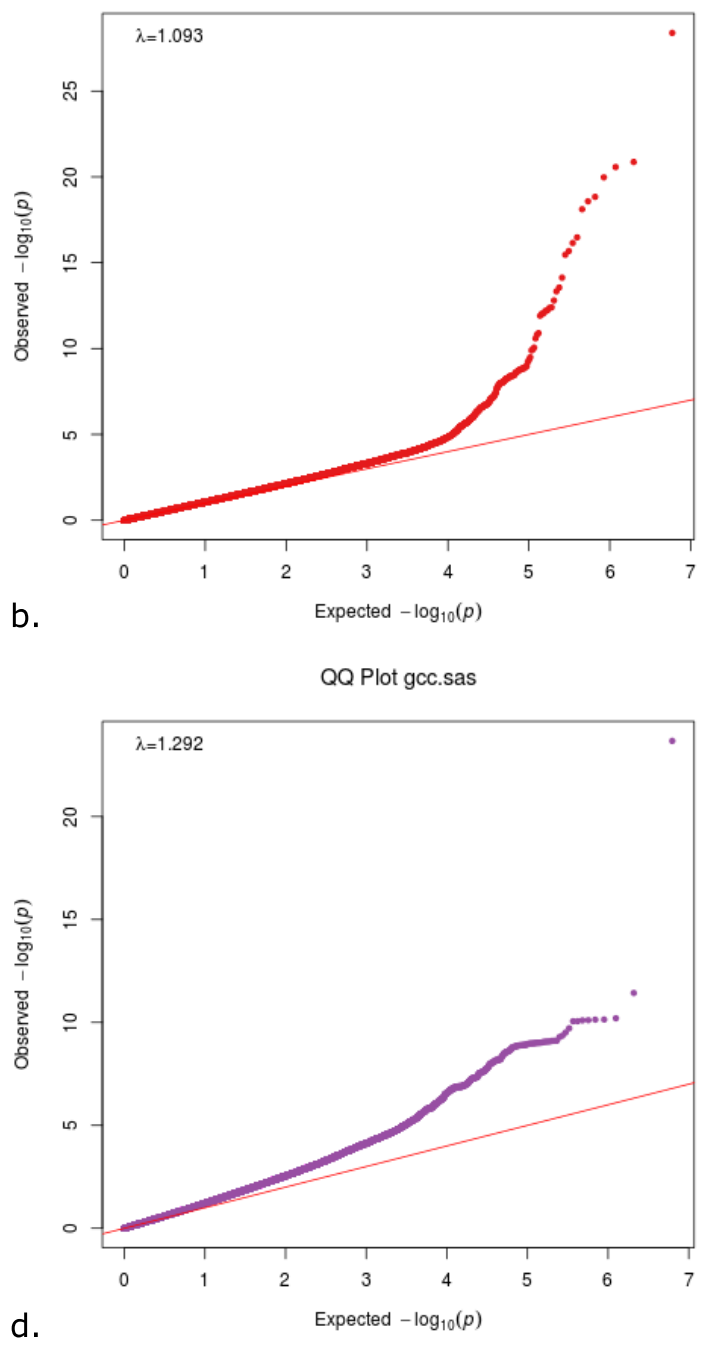

d.

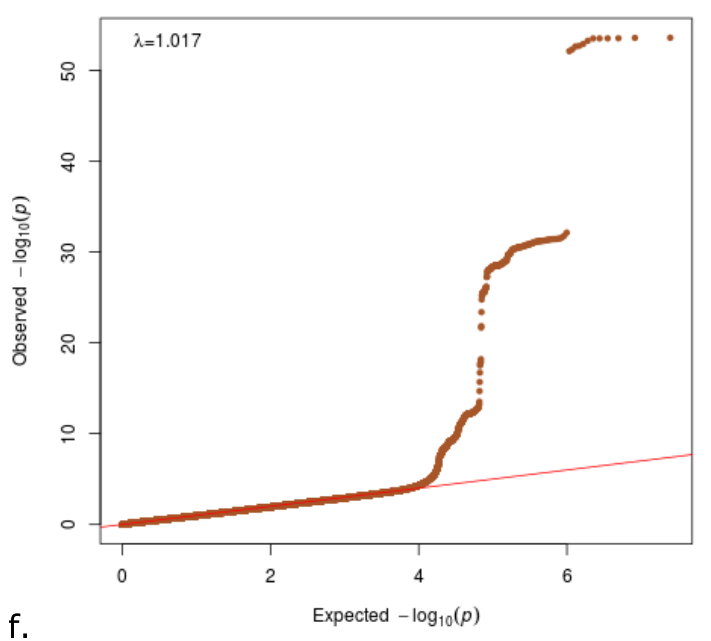

and 23andMe data (gcc.hgi. $23 \mathrm{~m}$ ). $\lambda$, genomic inflation value. Note that some residual inflation is evident in the primary analysis in GenOMICC European cohort. Repeating the analysis using more principal components (20 PCs) as covariates did not improve the inflation $\left(\lambda_{0.5}=1.10\right)$. 


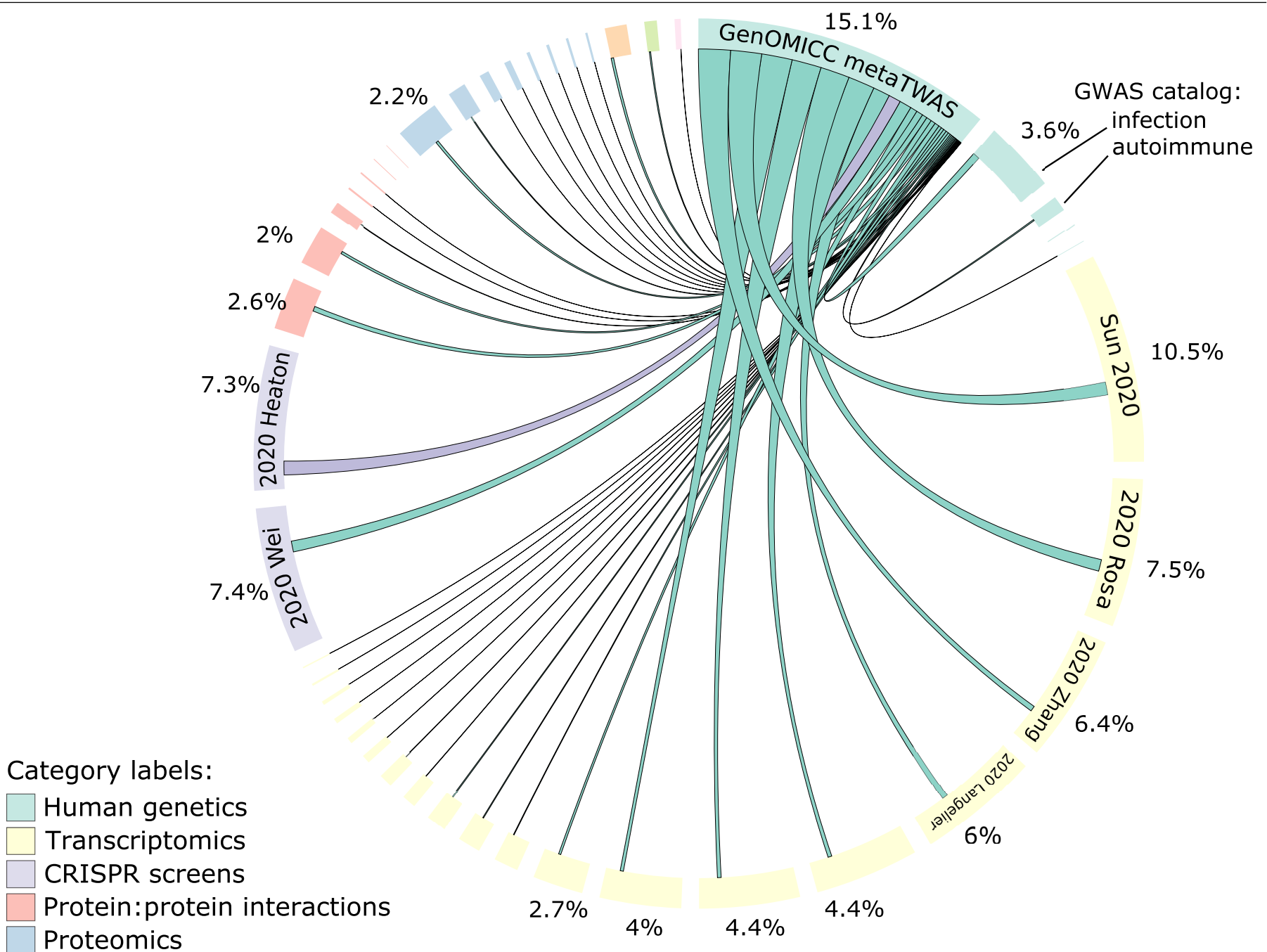

Extended Data Fig. 2 | MAIC shared information content. Representation of shared information content among data sources in the MAIC analysis. Each experiment or data source is represented by a block on the outer ring of the circle; the size of data source blocks is proportional to the summed information content of the input list-that is, the total contribution that this data source makes to the aggregate, calculated as the sum of the MAIC gene scores contributed by that list. Lines are coloured according to the dominant data source. Data sources within the same category share the same colour. The largest categories and data sources are labelled: Sun $2020^{79}, 2020$ Rosa $^{80}$,
2020 Zhang $^{81}, 2020$ Langelier $^{82}, 2020 \mathrm{Wei}^{83}, 2020$ Heaton $^{84}$. An interactive version of this figure is available at https://baillielab.net/maic/covid. To estimate the probability of the specific enrichment for GenOMICC metaTWAS, we randomly sampled genes from the baseline distribution of metaTWAS 1,000 times, re-running the MAIC with the same set of COVID-19 systematic review inputs, but substituting the randomly sampled input list for the GenOMICC metaTWAS results. Modelling a normal distribution based on these empirical results, we estimated that the probability of a MAIC enrichment with this strength arising by random chance is $P=4.2 \times 10^{-12}$. 


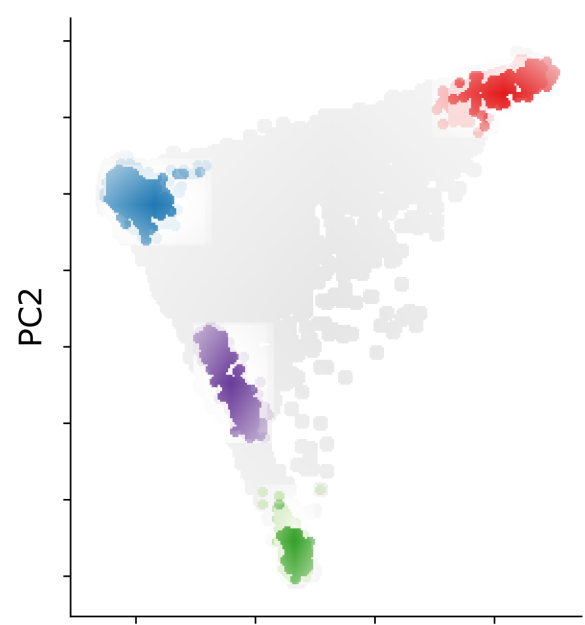

PC1
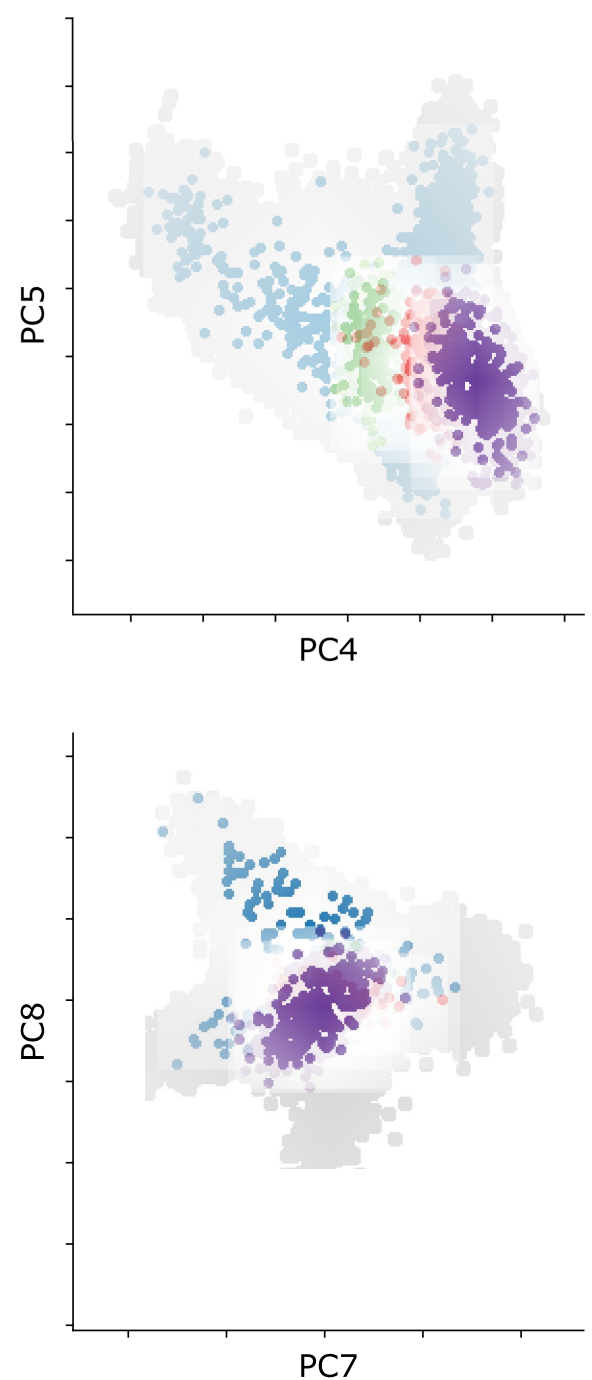
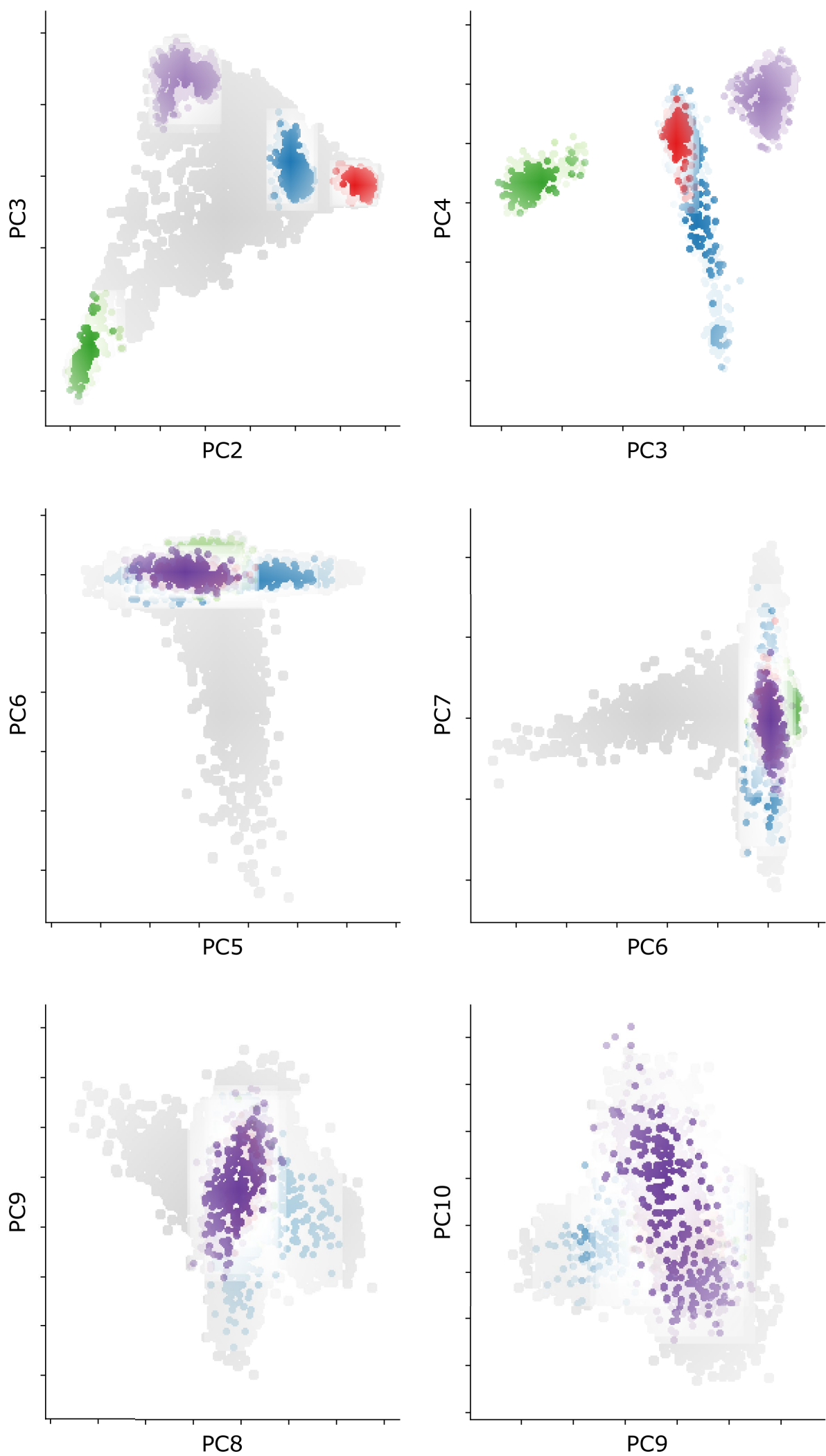

Extended Data Fig. 3 Genomic overlap among cases and controls. PCA plots show the distribution of all cases and controls for the first 10 principal components. Cases are shown as coloured circles: European (blue), African (red), East Asian (green) and South Asian (purple). Controls for each ancestry group are shown as circles in a lighter shade of the colour for that ancestry group. The UK Biobank data (background population) are shown as light-grey circles. 


\section{rs2236757 A/G}

\begin{tabular}{|c|c|c|c|c|}
\hline Cohort & Ratio & OR & $95 \%-\mathrm{Cl}$ & Weight \\
\hline gcc-AFR & & 1.08 & {$[0.76 ; 1.53]$} & $2.9 \%$ \\
\hline gcc-EAS & & 1.30 & {$[0.89 ; 1.91]$} & $2.4 \%$ \\
\hline gcc-SAS & $\Psi$ & 1.27 & {$[0.99 ; 1.62]$} & $5.9 \%$ \\
\hline gcc-EUR & + & 1.29 & {$[1.17 ; 1.41]$} & $43.0 \%$ \\
\hline $23 \mathrm{~m}$ & $\frac{1}{1}$ & 1.22 & {$[0.96 ; 1.55]$} & $6.3 \%$ \\
\hline hgi & $+\frac{1}{1}$ & 1.20 & {$[1.09 ; 1.32]$} & $39.7 \%$ \\
\hline Fixed effect model & 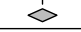 & 1.24 & {$[1.17 ; 1.32]$} & $100.0 \%$ \\
\hline Heterogeneity: $I^{2}=0 \%, \tau^{2}=0, p$ & & $\mathrm{p}$-val & $\mid=1.16 \mathrm{e}-12$ & \\
\hline
\end{tabular}

a.

\section{rs2109069 A/G}

Cohort

Odds Ratio

gcc-AFR

gCC-EAS

gCC-SAS

gCC-EUR

$23 \mathrm{~m}$

hgi

Fixed effect model Heterogeneity: $I^{2}=61 \%, \tau^{2}=0.0091, p=0.03$

c.

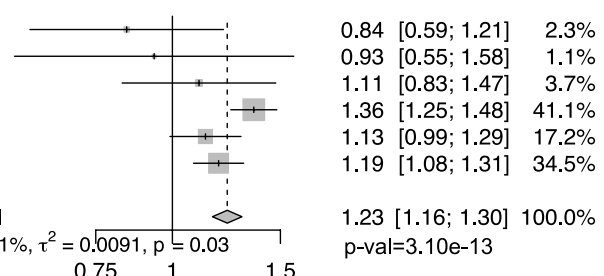

\section{rs73064425 T/C}

Cohort

Odds Ratio

OR

95\%-Cl Weight

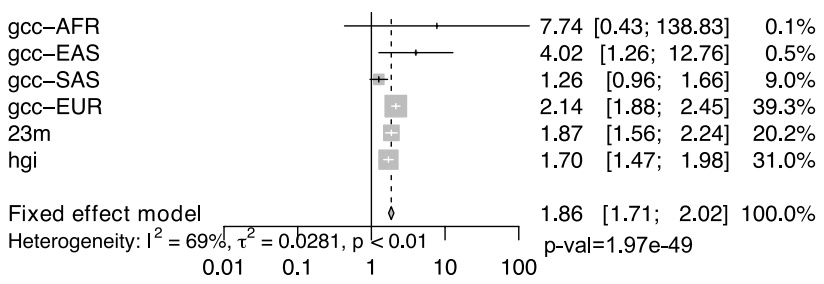

b.

rs10735079 G/A

Cohort Odds Ratio OR $95 \%-C l$ Weight

gCC-AFR

gcc-EAS

gCC-SAS

gCc-EUR

$23 m$

hgi

Fixed effect mode

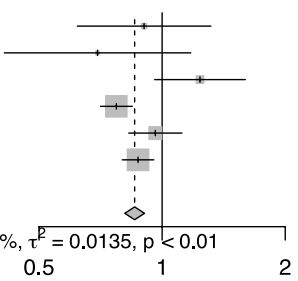

$0.90[0.62 ; 1.32] \quad 2.2 \%$ $0.69[0.41 ; 1.18] \quad 1.1 \%$ $1.24[0.96 ; 1.60] \quad 4.8 \%$ $0.77[0.71 ; 0.85] \quad 38.7 \%$ $0.96[0.83 ; 1.12] 13.8 \%$ $0.87[0.80 ; 0.95] \quad 39.4 \%$

$0.86[0.81 ; 0.91] 100.0 \%$ Heterogeneity: $I^{2}=69 \%, \tau^{\mathbb{P}=} 0.0135, \mathrm{p}<0.01$ $\mathrm{p}$-val $=5.04 \mathrm{e}-08$

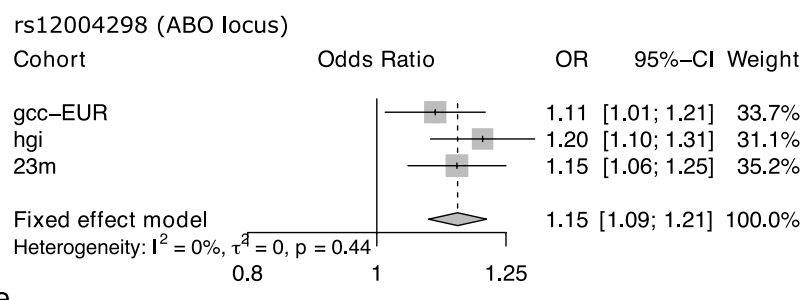

e.

Extended Data Fig. 4 | Effect sizes in ancestry groups within the GenoMICC study. a-e, Data are shown for the four replicated variants with genome-wide significant associations in GenOMICC (a-d) and the $A B O$ locus (e). Forest plots display the effect size heterogeneity measures and $P$ value (p) and meta-analysis estimates with $95 \%$ confidence interval $(\mathrm{Cl})$, and $P$ value (P-val) under a fixed-effect model. The allele in bold is the reference allele for the reported effect (odds ratio). Sample sizes for the cases and controls analysed in the four groups were: 1,092 individuals of African (AFR) ancestry, 894 individuals of East Asian (EAS) ancestry, 10,055 individuals of European (EUR) ancestry and 1,422 individuals of South Asian (SAS) ancestry within GenOMICC. HGI, COVID-19 HGI; 23m, 23andMe. Observed heterogeneity in the effect size may be owing to genuine differences between ancestry groups, the limited statistical power in smaller groups (evident from the broad confidence intervals) or residual confounding factors. 


\section{Article}

Extended Data Table 1 | Baseline characteristics of the 2,244 included patients

\begin{tabular}{|c|c|c|c|c|}
\hline \multirow[t]{2}{*}{ Patient Characteristics } & GenOMICC $(\mathrm{n}=2109)$ & \multicolumn{3}{|c|}{ ISARIC 4 C $(n=135)$} \\
\hline & & missing data & & missing data \\
\hline Female sex & $624(30 \%)$ & & $46(34 \%)$ & \\
\hline Age $(y r s$, mean \pm SD) & $57.3 \pm 12.1$ & & $57.3 \pm 2.9$ & \\
\hline European ancestry & $1573(75 \%)$ & & $103(76 \%)$ & \\
\hline South Asian ancestry & $219(10 \%)$ & & $18(13 \%)$ & \\
\hline African ancestry & $174(8 \%)$ & & $8(6 \%)$ & \\
\hline East Asian ancestry & $143(7 \%)$ & & $6(4 \%)$ & \\
\hline Significant comorbidity & $396(19 \%)$ & $49(2 \%)$ & $40(30 \%)$ & $26(19 \%)$ \\
\hline Invasive ventilation & $1557(74 \%)$ & $35(2 \%)$ & $25(19 \%)$ & $31(23 \%)$ \\
\hline Died (60 days) & $459(22 \%)$ & $338(16 \%)$ & $22(16 \%)$ & $30(22 \%)$ \\
\hline
\end{tabular}

Ancestry groups were determined by principal component analysis (Extended Data Fig. 3). Significant comorbidity was defined as the presence of functionally limiting comorbid illness in GenOMICC, according to the assessment of the treating clinicians. In ISARIC4C, significant comorbidity refers to the presence of any chronic cardiac, lung, kidney or liver disease, cancer or dementia. Age is shown as mean \pm standard deviation (SD). 
Extended Data Table 2 | Replication in external data

\begin{tabular}{lllllllll}
\hline SNP & chr:pos(b37) & Risk & Alt & OR $_{\text {gcc.ukb }}$ & $\mathrm{P}_{\text {gcc.ukb }}$ & OR $_{\text {hgi.23m }}$ & $\mathrm{P}_{\text {hgi.23m }}$ & Locus \\
\hline rs73064425 & $3: 45901089$ & $\mathrm{~T}$ & $\mathrm{C}$ & 2.1 & $4.8 \times 10^{-30}$ & 1.7 & $1.5 \times 10^{-28 *}$ & LZTFL1 \\
rs9380142 & $6: 29798794$ & $\mathrm{~A}$ & $\mathrm{G}$ & 1.3 & $3.2 \times 10^{-8}$ & 1 & 0.76 & HLA-G \\
rs143334143 & $6: 31121426$ & $\mathrm{~A}$ & $\mathrm{G}$ & 1.9 & $8.8 \times 10^{-18}$ & 1.1 & 0.019 & CCHCR1 \\
rs3131294 & $6: 32180146$ & $\mathrm{G}$ & $\mathrm{A}$ & 1.5 & $2.8 \times 10^{-8}$ & 0.99 & 0.91 & NOTCH4 \\
rs10735079 & $12: 113380008$ & $\mathrm{~A}$ & $\mathrm{G}$ & 1.3 & $1.6 \times 10^{-8}$ & 1.1 & $0.00082^{*}$ & OAS1/3 \\
rs2109069 & $19: 4719443$ & $\mathrm{~A}$ & $\mathrm{G}$ & 1.4 & $4 \times 10^{-12}$ & 1.1 & $5 \times 10^{-5 *}$ & DPP9 \\
rs74956615 & $19: 10427721$ & $\mathrm{~A}$ & $\mathrm{~T}$ & 1.6 & $2.3 \times 10^{-8}$ & 1.4 & $2 \times 10^{-6 *}$ & TYK2 \\
rs2236757 & $21: 34624917$ & $\mathrm{~A}$ & $\mathrm{G}$ & 1.3 & $5 \times 10^{-8}$ & 1.2 & $4.1 \times 10^{-5 *}$ & IFNAR2 \\
\hline
\end{tabular}

Alt, alternative allele; chr:pos, chromosome:position; locus, gene nearest to the top SNP; OR, effect size (odds ratio) of the risk allele; $P, P$ value; Risk, risk allele. Subscript identifiers show the data source: gcc.ukb, GenOMICC study, European ancestry, comparison with UK Biobank; hgi.23m, COVID-19 HGI and 23andMe meta-analysis, used for replication. Bonferroni significant values with external replication are indicated with an asterisk. 


\section{Reporting Summary}

Nature Research wishes to improve the reproducibility of the work that we publish. This form provides structure for consistency and transparency in reporting. For further information on Nature Research policies, see our Editorial Policies and the Editorial Policy Checklist.

\section{Statistics}

For all statistical analyses, confirm that the following items are present in the figure legend, table legend, main text, or Methods section.

n/a Confirmed

$\square$ The exact sample size $(n)$ for each experimental group/condition, given as a discrete number and unit of measurement

$\square$ A statement on whether measurements were taken from distinct samples or whether the same sample was measured repeatedly

$\square$ The statistical test(s) used AND whether they are one- or two-sided

$\square$ Only common tests should be described solely by name; describe more complex techniques in the Methods section.

$\square$ \ A description of all covariates tested

$\square$ \A description of any assumptions or corrections, such as tests of normality and adjustment for multiple comparisons

$\square$ A full description of the statistical parameters including central tendency (e.g. means) or other basic estimates (e.g. regression coefficient)

$\bigotimes$ AND variation (e.g. standard deviation) or associated estimates of uncertainty (e.g. confidence intervals)

$\varnothing$ For null hypothesis testing, the test statistic (e.g. $F, t, r$ ) with confidence intervals, effect sizes, degrees of freedom and $P$ value noted

Give P values as exact values whenever suitable.

Х $\square$ For Bayesian analysis, information on the choice of priors and Markov chain Monte Carlo settings

Х $\square$ For hierarchical and complex designs, identification of the appropriate level for tests and full reporting of outcomes

$\square \bigotimes$ Estimates of effect sizes (e.g. Cohen's d, Pearson's $r$ ), indicating how they were calculated

Our web collection on statistics for biologists contains articles on many of the points above.

\section{Software and code}

Policy information about availability of computer code

Data collection Illumina i-scan platform, GenomeStudio Analysis software v2.0.3, GSAMD-24v3-0-EA_20034606_A1.bpm manifest and cluster file provided by manufacturer

Data analysis GenomeStudio v2.03, DRAGEN v0.1.11.269.3.2.22, GATK 4.1.8.1, Plink 1.9, Plink 2.0, King 2.1, R v3.6, python v3.7, GATK 4.0, USC liftover, GCTA v1.92, SAIGE v0.39, metal, MAGMAv1.08, BCFtools 1.9, QCtools 1.3, FlashPCA2, admixture, FUMA v1.3.6, SMR/HEIDI v1.03, MetaXcan (git commit 0b7c10d633d3d7bfe794e4f35bd8190356bb2514), MiniMac4 v1.0,

For manuscripts utilizing custom algorithms or software that are central to the research but not yet described in published literature, software must be made available to editors and reviewers. We strongly encourage code deposition in a community repository (e.g. GitHub). See the Nature Research guidelines for submitting code \& software for further information.

\section{Data}

Policy information about availability of data

All manuscripts must include a data availability statement. This statement should provide the following information, where applicable:

- Accession codes, unique identifiers, or web links for publicly available datasets

- A list of figures that have associated raw data

- A description of any restrictions on data availability

Full summary-level data in support of the findings of this study are available for download from [https://genomicc.org/data](https://genomicc.org/data). Individual level data can be analysed by qualified researchers in the ISARIC 4C/GenOMICC data analysis platform by application at [https://genomicc.org/data](https:// genomicc.org/data).

The full GWAS summary statistics for the 23andMe discovery data set will be made available through 23andMe to qualified researchers under an agreement with 


\section{Field-specific reporting}

Please select the one below that is the best fit for your research. If you are not sure, read the appropriate sections before making your selection.

$\bigotimes$ Life sciences

$\square$ Behavioural \& social sciences

Ecological, evolutionary \& environmental sciences

For a reference copy of the document with all sections, see nature.com/documents/nr-reporting-summary-flat.pdf

\section{Life sciences study design}

All studies must disclose on these points even when the disclosure is negative.

Sample size $\quad n=2244$ critically ill Covid-19 patients, $n=11220$ random controls matched by ancestry from UK Biobank. The sample size was determined pragmatically by the number of cases recruited during the first wave of the outbreak in the UK. Adequate statistical power was determined by the detection of significant associations, and is confirmed by replication in external studies.

Data exclusions Patients of mixed genetic ancestry, and from ancestry groups with small numbers of cases (such as North American Indian) defined using principal components analysis, were excluded because we were not able to match adequate controls for these individuals.

Replication $\quad$ Replicated main findings using 2415 hospitalised Covid-19 patients and 477741 population controls from Covid19 Host genetics initiative and 1128 Covid19 cases and 679531 population controls from 23andme Inc "broad respiratory" phenotype. 3 variants did not replicate; all are in the $\mathrm{MHC}$ region, which is both highly sensitive to population stratification (potentially causing spurious associations) and commonly associated with infectious and immune disease.

Randomization Not relevant to the study. There wasn't any allocation to experimental groups

Blinding Blinding was not used in this study because the exposure (genotype) and outcome (ICU admission) are objective. Confounding was controlled by the use of covariates: age, sex, deprivation score and genetic ancestry (principal components).

\section{Reporting for specific materials, systems and methods}

We require information from authors about some types of materials, experimental systems and methods used in many studies. Here, indicate whether each material, system or method listed is relevant to your study. If you are not sure if a list item applies to your research, read the appropriate section before selecting a response.

Materials \& experimental systems

\begin{tabular}{l|l}
\multicolumn{2}{l}{ Methods } \\
\hline n/a & Involved in the study \\
\hline & $\square$ ChIP-seq \\
$\triangle$ & $\square$ Flow cytometry \\
$\square$ & $\square$ MRI-based neuroimaging
\end{tabular}

n/a Involved in the study

Х Antibodies

Х Eukaryotic cell lines

Х $\square$ Palaeontology and archaeology

\ $\square$ Animals and other organisms

$\square$ Human research participants

\ $\square$ Clinical data

\ Dual use research of concern

\section{Human research participants}

Policy information about studies involving human research participants

Population characteristics

Recruitment

Ethics oversight
1574 males, 670 females. 1176 European ancestry, 227 South Asian ancestry, 182 African ancestry, 149 East Asian ancestry. Mean age is 57.3 years

Critically-ill cases were recruited through the GenOMICC study in 208 UK Intensive Care Units and hospitalised cases through the International Severe Acute Respiratory Infection Consortium (ISARIC) Coronavirus Clinical Characterisation Consortium (4C) study. Genomicc patients ha confirmed Covid-19 according to local clinical testing and were deemed by the treating physician to require continuous cardiorespiratory monitoring in intensive care units. ISARIC4C individuals had confirmed Covid-19 and were deemed to require hospital admission. Since this outcome is determined by clinicians it is unlikely to be affected by self-selection bias.

Research ethics committees (Scotland 15/SS/0110, England, Wales and Northern Ireland: 19/WM/0247. Current and previous versions of the study protocol are available at genomicc.org/protocol. All participants gave informed consent. 